\title{
A proximal method for solving nonlinear minmax location problems with perturbed minimal time functions via conjugate duality
}

\author{
Sorin-Mihai Grad ${ }^{1}$ id $\cdot$ Oleg Wilfer ${ }^{2}$
}

Received: 10 April 2018 / Accepted: 23 January 2019 / Published online: 8 February 2019

(C) The Author(s) 2019

\begin{abstract}
We investigate via a conjugate duality approach general nonlinear minmax location problems formulated by means of an extended perturbed minimal time function, necessary and sufficient optimality conditions being delivered together with characterizations of the optimal solutions in some particular instances. A parallel splitting proximal point method is employed in order to numerically solve such problems and their duals. We present the computational results obtained in MATLAB on concrete examples, successfully comparing these, where possible, with earlier similar methods from the literature. Moreover, the dual employment of the proximal method turns out to deliver the optimal solution to the considered primal problem faster than the direct usage on the latter. Since our technique successfully solves location optimization problems with large data sets in high dimensions, we envision its future usage on big data problems arising in machine learning.
\end{abstract}

Keywords Gauge (Minkowski) function · Minimal time function · Minmax multifacility location problem - Sylvester problem · Apollonius problem · Proximal point algorithm · Epigraphical projection $\cdot$ Projection operator $\cdot$ Machine learning

\section{Preliminaries}

In this paper we investigate nonlinear minmax location problems that are generalizations of the classical Sylvester problem in location theory—not to be confused with Sylvester's line

Sorin-Mihai Grad: Formerly at the Faculty of Mathematics and Computer Science, Leipzig University; partially supported by DFG (the German Research Foundation) under Project GR3367/4-1 and FWF (Austrian Science Fund), Project M-2045.

Sorin-Mihai Grad

sorin-mihai.grad@univie.ac.at

Oleg Wilfer

oleg.wilfer@mathematik.tu-chemnitz.de

1 Faculty of Mathematics, University of Vienna, Oskar-Morgenstern-Platz 1, 1090 Vienna, Austria

2 Faculty of Mathematics, Chemnitz University of Technology, 09107 Chemnitz, Germany 
problem, and of the celebrated Apollonius problem, formulated by means of an extended perturbed minimal time function via a conjugate duality approach, necessary and sufficient optimality conditions being delivered together with characterizations of the optimal solutions in some particular instances. This approach is necessary in order to be able to numerically solve such problems and their corresponding dual problems by means of a proximal method. Applying the mentioned algorithm on the duals of some concrete location problems in MAT$\mathrm{LAB}$ delivers optimal solutions to the latter faster and with reduced costs. To the best of our knowledge this is the first time such a method is considered for this type of location optimization problems and the general framework we consider opens the possibility for other problems of more or less similar type (arising, for instance, in machine learning) to be solved in an analogous way. The original location optimization problem cannot be directly numerically solved by means of the usual algorithms because the involved functions often lack differentiability, while a direct employment of some proximal point method is not possible because of the complicated structure of the objective function, that consists of the maximum of $n$ functions, each containing a composition of functions.

In order to introduce the general nonlinear minmax location problems we propose a new perturbed minimal time function that generalizes the classical minimal time function, introduced over 4 decades ago and recently reconsidered by Mordukhovich and Nam in a series of papers (see, for instance, [14-16,19]) and the book [13], and several of its recent extensions (cf. $[12,16,19,24,31])$. The motivation to investigate such problems comes from both theoretical and practical reasons, as location type problems arise in various areas of research and real life, such as geometry, physics, economics or health management, applications from these fields being mentioned in our paper as possible interpretations of our results. As suggested, for instance, in $[1,21]$, solving general location problems as considered in this paper could prove to be useful in dealing with some classes of constrained optimization, too, like the ones that appear in machine learning. Actually, given the fact that the algorithm we propose is able to successfully solve location optimization problems with large data sets in high dimensions faster than its counterparts from the literature makes us confident regarding a future usage of this technique on big data problems arising in machine learning, for instance those approached by means of support vector techniques.

To be able to deal with the considered general nonlinear minmax location problems by means of conjugate duality we rewrite them as multi-composed optimization problems, for which we have recently proposed a duality approach in $[10,25,27,29]$. The corresponding necessary and sufficient optimality conditions are then derived. While most of the theoretical results are provided in the general framework of Banach spaces, in the more restrictive setting of Hilbert spaces we were also able to provide characterizations of the optimal solutions of the considered problems by means of the dual optimal solutions. Two special cases of the general problem, motivated by economic interpretations, are discussed in a more detailed way, followed by an exact formula of the projection operator onto the epigraph of the maximum of norms, that may prove to be useful in other applications, too. The fourth section of the paper is dedicated to numerical experiments that are presented in a finitely dimensional framework that is specific to most of the possible practice applications of our results. Employing a splitting proximal point method from [3], we solve in MATLAB concrete location optimization problems corresponding to the mentioned special cases and their conjugate duals, rewritten as unconstrained minimization problems. The computational results show that the primal optimal solutions are obtained faster when numerically solving the dual problems. One of these concrete examples was numerically solved in $[13,17]$ by means of a subgradient method and a comparison of the computational results is provided as well, stressing once again the superiority of the algorithm proposed in the present paper. Another comparison is made 
with the log-exponential smoothing accelerated gradient method proposed in [1] in several examples, one of them including a large data set in high dimensions and our method turns out again to converge faster towards the optimal solution of the considered location problem.

Let $X$ be a Hausdorff locally convex space and $X^{*}$ its topological dual space endowed with the weak* topology $w\left(X^{*}, X\right)$. Thus the dual of $X^{*}$ is $X$. For $x \in X$ and $x^{*} \in X^{*}$, let $\left\langle x^{*}, x\right\rangle:=x^{*}(x)$ be the value of the linear continuous functional $x^{*}$ at $x$. A set $U \subseteq X$ is called convex if $t x+(1-t) y \in U$ for all $x, y \in U$ and $t \in[0,1]$. A nonempty set $K \subseteq X$ that satisfies the condition $t K \subseteq K$ for all $t \geq 0$ is said to be a cone. Note that any cone contains the origin of the space it lies in, denoted by $0_{X}$ for the space $X$. Consider a convex cone $K \subseteq X$, which induces on $X$ a partial ordering relation " $\leqq_{K}$ ", defined by $\leqq_{K}:=\{(x, y) \in X \times X: y-x \in K\}$, i.e. for $x, y \in X$ it holds $x \leqq_{K} y \Leftrightarrow y-x \in K$. We attach to $X$ a largest element with respect to " $\leqq_{K}$ ", denoted by $+\infty_{K}$, which does not belong to $X$ and denote $\bar{X}=X \cup\left\{+\infty_{K}\right\}$. Then it holds $x \leqq_{K}+\infty_{K}$ for all $x \in \bar{X}$. We write $x \leq_{K} \quad y$ when $x \leqq_{K} \quad y$ and $x \neq y, \leq:=\leqq_{\mathbb{R}_{+}}$and $<:=\leq_{\mathbb{R}_{+}}$. On $\bar{X}$ consider the following operations and conventions: $x+\left(+\infty_{K}\right)=\left(+\infty_{K}\right)+x:=+\infty_{K}$ for all $x \in \bar{X}$ and $\lambda \cdot\left(+\infty_{K}\right):=+\infty_{K}$ for all $\lambda \in[0,+\infty] . K^{*}:=\left\{x^{*} \in X^{*}:\left\langle x^{*}, x\right\rangle \geq 0\right.$ $\forall x \in K\}$ is the dual cone of $K$ and by convention $\left\langle x^{*},+\infty \infty_{K}\right\rangle:=+\infty$ for all $x^{*} \in K^{*}$. By a slight abuse of notation we denote the extended real space $\overline{\mathbb{R}}=\mathbb{R} \cup\{ \pm \infty\}$ and consider on it the following operations and conventions: $\lambda+(+\infty)=(+\infty)+\lambda:=+\infty$ for all $\lambda \in[-\infty,+\infty], \lambda+(-\infty)=(-\infty)+\lambda:=-\infty$ for all $\lambda \in[-\infty,+\infty), \lambda \cdot(+\infty):=+\infty$ for all $\lambda \in[0,+\infty], \lambda \cdot(+\infty):=-\infty$ for all $\lambda \in[-\infty, 0), \lambda \cdot(-\infty):=-\infty$ for all $\lambda \in(0,+\infty], \lambda \cdot(-\infty):=+\infty$ for all $\lambda \in[-\infty, 0)$ and $0(-\infty):=0$. Given $S \subseteq X$, we denote its algebraic interior by core $S$, its normal cone at $x \in X$ is $N_{S}(x):=\left\{x^{*} \in\right.$ $\left.X^{*}:\left\langle x^{*}, y-x\right\rangle \leq 0 \forall y \in S\right\}$ if $x \in S$ and $N_{S}(x)=\emptyset$ otherwise, its conic hull is cone $S:=\{\lambda x: x \in S, \lambda \geq 0\}$, while if $S$ is convex its strong quasi relative interior (see [6]) is sqri $S:=\{x \in S: \operatorname{cone}(S-x)$ is a closed linear subspace $\}$.

For a given function $f: X \rightarrow \overline{\mathbb{R}}$ we consider its effective domain $\operatorname{dom} f:=\{x \in$ $X: f(x)<+\infty\}$ and call $f$ proper if $\operatorname{dom} f \neq \emptyset$ and $f(x)>-\infty$ for all $x \in X$. The epigraph of $f$ is epi $f=\{(x, r) \in X \times \mathbb{R}: f(x) \leq r\}$. Recall that a function $f: X \rightarrow \overline{\mathbb{R}}$ is called convex if $f(\lambda x+(1-\lambda) y) \leq \lambda f(x)+(1-\lambda) f(y)$ for all $x, y \in X$ and all $\lambda \in[0,1]$. For a subset $A \subseteq X$, its indicator function $\delta_{A}: X \rightarrow \overline{\mathbb{R}}$ is

$$
\delta_{A}(x):= \begin{cases}0, & \text { if } x \in A, \\ +\infty, & \text { otherwise, }\end{cases}
$$

and its support function $\sigma_{A}: X^{*} \rightarrow \overline{\mathbb{R}}$ is $\sigma_{A}\left(x^{*}\right)=\sup _{x \in A}\left\langle x^{*}, x\right\rangle$. The conjugate function of $f$ with respect to the nonempty subset $S \subseteq X$ is defined by

$$
f_{S}^{*}: X^{*} \rightarrow \overline{\mathbb{R}}, \quad f_{S}^{*}\left(x^{*}\right)=\sup _{x \in S}\left\{\left\langle x^{*}, x\right\rangle-f(x)\right\} .
$$

One has the Young-Fenchel inequality $f(x)+f_{S}^{*}\left(x^{*}\right) \geq\left\langle x^{*}, x\right\rangle$ for all $x \in S$ and all $x^{*} \in X^{*}$. In the case $S=X, f_{S}^{*}$ turns into the classical Fenchel-Moreau conjugate function of $f$ denoted by $f^{*}$. The conjugate of $f^{*}$ is said to be the biconjugate function of $f$ and is denoted by $f^{* *}: X \rightarrow \overline{\mathbb{R}}$. Given the proper functions $f_{i}: X \rightarrow \overline{\mathbb{R}}, i=1, \ldots, n$, their infimal convolution is $f_{1} \square f_{2} \square \ldots \square f_{n}: X \rightarrow \overline{\mathbb{R}},\left(f_{1} \square f_{2} \square \ldots \square f_{n}\right)(x)=\inf \left\{\sum_{i=1}^{n} f_{i}\left(x_{i}\right): x_{i} \in\right.$ $\left.X, i=1, \ldots, n, \sum_{i=1}^{n} x_{i}=x\right\}$. We say that the infimal convolution is exact at $x \in X$ when for $x$ the infimum in its definition is attained. 


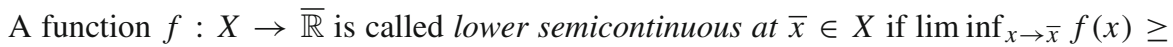
$f(\bar{x})$ and when this function is lower semicontinuous at all $x \in X$, then we call it lower semicontinuous. The largest lower semicontinuous function nowhere larger than $f$ is its lower semicontinuous envelope $\bar{f}: X \rightarrow \overline{\mathbb{R}}$. Let $W \subseteq X$ be a nonempty set, then a function $f$ : $X \rightarrow \overline{\mathbb{R}}$ is called $K$-increasing on $W$, if from $x \leqq_{K} y$ follows $f(x) \leq f(y)$ for all $x, y \in W$. When $W=X$, then we call the function $f K$-increasing. If we take an arbitrary $x \in X$ such that $f(x) \in \mathbb{R}$, then we call the set $\partial f(x):=\left\{x^{*} \in X^{*}: f(y)-f(x) \geq\left\langle x^{*}, y-x\right\rangle \forall y \in X\right\}$ the (convex) subdifferential of $f$ at $x$, where the elements of this set are called subgradients. Moreover, if $\partial f(x) \neq \varnothing$, then we say that $f$ is subdifferentiable at $x$ and if $f(x) \notin \mathbb{R}$, then we make the convention that $\partial f(x):=\emptyset$. Note that the subgradients of $f$ can be characterized by means of $f^{*}$, more precisely $x^{*} \in \partial f(x)$ if and only if $f(x)+f^{*}\left(x^{*}\right)=\left\langle x^{*}, x\right\rangle$, i.e. the Young-Fenchel inequality is fulfilled as an equality for $x$ and $x^{*}$.

Let $Z$ be another Hausdorff locally convex space partially ordered by the convex cone $Q \subseteq$ $Z$ and $Z^{*}$ its topological dual space endowed with the weak* topology $w\left(Z^{*}, Z\right)$. The domain of a vector function $F: X \rightarrow \bar{Z}=Z \cup\left\{+\infty_{Q}\right\}$ is $\operatorname{dom} F:=\left\{x \in X: F(x) \neq+\infty_{Q}\right\} . F$ is called proper if dom $F \neq \emptyset$. When $F(\lambda x+(1-\lambda) y) \leqq Q \lambda F(x)+(1-\lambda) F(y)$ holds for all $x, y \in X$ and all $\lambda \in[0,1]$ the function $F$ is said to be $Q$-convex. The $Q$-epigraph of $F$ is epi $_{Q} F=\{(x, z) \in X \times Z: F(x) \leqq Q z\}$ and when $Q$ is closed we say that $F$ is $Q$-epi-closed if epi $\mathrm{i}_{Q} F$ is a closed set. Let us mention that in the case $Z=\mathbb{R}$ and $Q=\mathbb{R}_{+}$, the notion of $Q$-epi-closedness falls into the one of lower semicontinuity. For a $z^{*} \in Q^{*}$ we define the function $\left(z^{*} F\right): X \rightarrow \overline{\mathbb{R}}$ by $\left(z^{*} F\right)(x):=\left\langle z^{*}, F(x)\right\rangle$. Then $\operatorname{dom}\left(z^{*} F\right)=\operatorname{dom} F$. Moreover, it is easy to see that if $F$ is $Q$-convex, then $\left(z^{*} F\right)$ is convex for all $z^{*} \in Q^{*}$. Let us point out that by the operations we defined on a Hausdorff locally convex space attached with a maximal element and on the extended real space, there holds $0 f=\delta_{\operatorname{dom} f}$ and $\left(0_{Z^{*}} F\right)=\delta_{\operatorname{dom} F}$ for any $f: X \rightarrow \overline{\mathbb{R}}$ and $F: X \rightarrow \bar{Z}$. The vector function $F$ is called positively $Q$-lower semicontinuous at $x \in X$ if $\left(z^{*} F\right)$ is lower semicontinuous at $x$ for all $z^{*} \in Q^{*}$. The function $F$ is called positively $Q$-lower semicontinuous if it is positively $Q$-lower semicontinuous at every $x \in X$. Note that if $F$ is positively $Q$-lower semicontinuous, then it is also $Q$ epi-closed, while the inverse statement is not true in general (see: [6, Proposition 2.2.19]). $F: X \rightarrow \bar{Z}$ is called $(K, Q)$-increasing on $W$, if from $x \leqq_{K} y$ follows $F(x) \leqq_{Q} F(y)$ for all $x, y \in W$. When $W=X$, we call this function $(K, Q)$-increasing. Last but not least denote the optimal objective value of an optimization problem $(P)$ by $v(P)$ and note that when an infimum/supremum is attained we write $\mathrm{min} / \mathrm{max}$ instead of inf/sup.

Furthermore, let $\mathcal{H}$ be a real Hilbert space equipped with the scalar product $\langle\cdot, \cdot\rangle_{\mathcal{H}}$, where the associated norm $\|\cdot\|_{\mathcal{H}}$ is defined by $\|y\|_{\mathcal{H}}:=\sqrt{\langle y, y\rangle_{\mathcal{H}}}$ for all $y \in \mathcal{H}$. If $\mathcal{H}=\mathbb{R}^{m}$, then $\|\cdot\|_{\mathbb{R}^{m}}$ is the Euclidean norm and we will write for simplicity just $\|\cdot\|$. The proximal point operator of parameter $\gamma>0$ of a function $f: \mathcal{H} \rightarrow \overline{\mathbb{R}}$ at $x \in \mathcal{H}$ is defined as

$$
\operatorname{prox}_{\gamma f}: \mathcal{H} \rightarrow \mathcal{H}, \operatorname{prox}_{\gamma f}(x)=\underset{y \in \mathcal{H}}{\arg \min }\left\{\gamma f(y)+\frac{1}{2}\|y-x\|^{2}\right\} .
$$

For more on convex optimization in Hilbert spaces we warmly recommend [3].

\section{Nonlinear minmax location problems}

As the perturbed minimal time functions play a decisive role in this article, we start this section with some of their properties. 


\subsection{Properties of the perturbed minimal time function}

In order to introduce the perturbed minimal time functions, one needs first to define a gauge. In the literature one can find different functions called gauges, see, for instance, [9] or [22, Section 15]. In the following we call gauge function (known in the literature also as the Minkowski functional) of a set $C \subseteq X$ the function $\gamma_{C}: X \rightarrow \overline{\mathbb{R}}$, defined by

$$
\gamma_{C}(x):=\inf \{\lambda>0: x \in \lambda C\} .
$$

Note that the gauge function can also take the value $+\infty$ if there does not exists an element $\lambda>0$ such that $x \in \lambda C$, as by definition it holds inf $\emptyset=+\infty$. From the definition it follows that dom $\gamma_{C}=$ cone $C$ if $0 \in C$ and dom $\gamma_{C}=$ cone $C \backslash\{0\}$ if $0 \notin C$. As $\gamma_{\emptyset} \equiv+\infty$, we consider further the set $C$ to be nonempty. The conjugate function of $\gamma_{C}$ is (cf. [6, Example 2.3.4], as the additional hypotheses imposed there on $C$ are actually not employed for this formula) $\gamma_{C}^{*}: X^{*} \rightarrow \overline{\mathbb{R}}$

$$
\gamma_{C}^{*}\left(x^{*}\right)= \begin{cases}0, & \text { if } \sigma_{C}\left(x^{*}\right) \leq 1, \\ +\infty, & \text { otherwise. }\end{cases}
$$

Furthermore, the polar set of $C$ is $C^{0}:=\left\{x^{*} \in X^{*}: \sigma_{C}\left(x^{*}\right) \leq 1\right\}$, and by means of the polar set the dual gauge of $C$ is defined by

$$
\gamma_{C^{0}}\left(x^{*}\right):=\sup _{x \in C}\left\langle x^{*}, x\right\rangle=\sigma_{C}\left(x^{*}\right) .
$$

Remark 2.1 The conjugate of $\gamma_{C}$ can equivalently be expressed by

$$
\gamma_{C}^{*}\left(x^{*}\right):=\left\{\begin{array}{ll}
0, & \text { if } \gamma_{C^{0}}\left(x^{*}\right) \leq 1, \\
+\infty, & \text { otherwise, }
\end{array}=\delta_{C^{0}}\left(x^{*}\right) \forall x^{*} \in X^{*} .\right.
$$

It is well-known that for the gauge function the generalized Cauchy-Schwarz inequality is fulfilled. The proof of this inequality is rare to find and for this reason we give it here.

Lemma 2.1 It holds $\gamma_{C}(x) \gamma_{C^{0}}\left(x^{*}\right) \geq\left\langle x^{*}, x\right\rangle$ for all $x \in X, x^{*} \in X^{*}$.

Proof Let $x^{*} \in X^{*}$ and $x \in X$. If $\left\langle x^{*}, x\right\rangle \leq 0$, then there is nothing to prove, as the gauge and the dual gauge are nonnegative functions.

Let $\left\langle x^{*}, x\right\rangle>0$. If $\gamma_{C}(x)>0$ then we have that

$$
\gamma_{C^{0}}\left(x^{*}\right) \geq \sup _{\lambda>0, \gamma_{C}(x) \leq \frac{1}{\lambda}} \lambda\left\langle x^{*}, x\right\rangle=\left\langle x^{*}, x\right\rangle \sup _{0<\lambda \leq \frac{1}{\gamma_{C}(x)}} \lambda=\frac{1}{\gamma_{C}(x)}\left\langle x^{*}, x\right\rangle,
$$

i.e. $\gamma_{C}(x) \gamma_{C^{0}}\left(x^{*}\right) \geq\left\langle x^{*}, x\right\rangle$.

Otherwise, if $\gamma_{C}(x)=0$, then one has that

$$
\gamma_{C^{0}}(x) \geq \sup _{\lambda>0,0=\gamma_{C}(x) \leq \frac{1}{\lambda}} \lambda\left\langle x^{*}, x\right\rangle=+\infty,
$$

i.e. $\gamma_{C}(x) \gamma_{C^{0}}\left(x^{*}\right)=0(+\infty) \geq\left\langle x^{*}, x\right\rangle$.

Given a nonempty set $\Omega \subset X$ and a proper function $f: X \rightarrow \overline{\mathbb{R}}$, we define the extended perturbed minimal time function $\mathcal{T}_{\Omega, f}^{C}: X \rightarrow \overline{\mathbb{R}}$ as the infimal convolution of $\gamma_{C}, f$ and $\delta_{\Omega}$, i.e. $\mathcal{T}_{\Omega, f}^{C}:=\gamma_{C} \square f \square \delta_{\Omega}$, more precisely

$$
\mathcal{T}_{\Omega, f}^{C}(x):=\inf _{y \in X, z \in \Omega}\left\{\gamma_{C}(x-y-z)+f(y)\right\} .
$$


Remark 2.2 To the best of our knowledge the function $\mathcal{T}_{\Omega, f}^{C}$ has not been considered in this form in the literature yet and it covers as special cases several important functions. For instance, if $f=\delta_{\left\{0_{X}\right\}}$, then one gets the classical minimal time function (see [13-15]) $\mathcal{T}_{\Omega}^{C}: \mathbb{R}^{n} \rightarrow \overline{\mathbb{R}}, \mathcal{T}_{\Omega}^{C}(x):=\inf \{t \geq 0:(x-t C) \cap \Omega \neq \emptyset\}$, that, when $\Omega=\left\{0_{X}\right\}$ collapses to the gauge function. In [12] one finds two perturbations of the classical minimal time function, $\gamma_{C} \square f$ (introduced in [31] and motivated by a construction specific to differential inclusions) and $\gamma_{C} \square\left(f+\delta_{\Omega}\right)$, that contains as a special case the perturbed distance function introduced in [24]. The latter function has motivated us to introduce $\mathcal{T}_{\Omega, f}^{C}$, where the function $f$ and the set $\Omega$ do not share the same variable anymore and can thus be split in the dual representations. Other generalizations of the classical minimal time function can be found, for instance, in $[5,16,19]$. The minimal time function and its generalizations have been employed in various areas of research such as location theory (cf. $[13,19]$ ), nonsmooth analysis (cf. $[5,12,14$ $16,19,24,31]$ ), control theory and Hamilton-Jacobi partial differential equation (mentioned in [5]), best approximation problems (cf. [24]) and differential inclusions (cf. [31]). Note also the connection observed in [19] between the minimal time function and the scalarization function due to Tammer (Gerstewitz) considered in vector optimization.

Moreover, as $\mathcal{T}_{\Omega, f}^{C}$ is an infimal convolution its conjugate function turns into (see [6, Proposition 2.3.8.(b)] $\left(\mathcal{T}_{\Omega, f}^{C}\right)^{*}=\gamma_{C}^{*}+f^{*}+\sigma_{\Omega}=\delta_{C^{0}}+f^{*}+\sigma_{\Omega}$ (see Remark 2.1).

Since dom $\gamma_{C} \neq \emptyset$ and $\gamma_{C}$ is a nonnegative function, it follows by [6, Lemma 2.3.1.(b)] that $\gamma_{C}^{*}=\delta_{C^{0}}$ is proper, convex and lower semicontinuous. If $f$ has an affine minorant, then $f^{*}$ is a proper, convex and lower semicontinuous function, too. Further, under the additional assumption $C^{0} \cap \operatorname{dom} f^{*} \cap \operatorname{dom} \sigma_{\Omega} \neq \emptyset,[6$, Theorem 2.3.10] yields that the biconjugate of $\mathcal{T}_{\Omega, f}^{C}$ is, under the mentioned hypotheses, given by $\left(\mathcal{T}_{\Omega, f}^{C}\right)^{* *}=\overline{\gamma_{C}^{* *} \square f^{* *} \square \sigma_{\Omega}^{*}}$, and one can derive as byproducts conjugate and biconjugate formulae for the classical minimal time function and its other extensions mentioned in Remark 2.2.

Theorem 2.1 Let $C$ be convex, closed and contain $0_{X}, \Omega$ be closed and convex and $f: X \rightarrow$ $\overline{\mathbb{R}}$ be also convex and lower semicontinuous such that $C^{0} \cap \operatorname{dom} f^{*} \cap \operatorname{dom} \sigma_{\Omega} \neq \emptyset$. Suppose that one of the following holds

(a) epi $\gamma_{C}+$ epi $f+\left(\Omega \times \mathbb{R}_{+}\right)$is closed,

(b) there exists an element $x^{*} \in C^{0} \cap \operatorname{dom} f^{*} \cap \operatorname{dom} \sigma_{\Omega}$ such that two of the functions $\delta_{C^{0}}, f^{*}$ and $\sigma_{\Omega}$ are continuous at $x^{*}$.

Then $\mathcal{T}_{\Omega, f}^{C}$ is proper, convex and lower semicontinuous and moreover, it holds

$$
\mathcal{T}_{\Omega, f}^{C}(x)=\min _{y \in X, z \in \Omega}\left\{\gamma_{C}(x-y-z)+f(y)\right\} \quad \forall x \in X,
$$

i.e. the infimal convolution of $\gamma_{C}, f$ and $\delta_{\Omega}$ is exact.

Proof As $C$ is closed and convex such that $0_{X} \in C$, it follows by [27, Theorem 1] that $\gamma_{C}$ is proper, convex and lower semicontinuous. Further, the nonemptiness, closedness and convexity of $\Omega$ imply the properness, convexity and lower semicontinuity of $\delta_{\Omega}$. Hence, one gets from the Fenchel-Moreau Theorem that $\gamma_{C}^{* *}=\gamma_{C}, f^{* *}=f$ and $\delta_{\Omega}^{* *}=\delta_{\Omega}$ and from [6, Theorem 3.5.8.(a)], taking into consideration that the conjugate functions of $f, \delta_{\Omega}$ and $\gamma_{C}$ are proper, convex and lower semicontinuous (as noted above), follows the desired statement. $\square$

Remark 2.3 Under the hypotheses of Theorem 2.1 the subdifferential of $\mathcal{T}_{\Omega, f}^{C}$ can be written for any $x \in X$ as $\partial \mathcal{T}_{\Omega, f}^{C}(x)=\partial \gamma_{C}(x-y-z) \cap \partial f(y) \cap N_{\Omega}(z)$, where $y$ and $z$ are the points where the minimum in the definition of the infimal convolution is attained. Note, moreover, 
that the subdifferential of $\gamma_{C}$ at any $x \in X$ coincides with the face of $C^{0}$ exposed by $x$ (cf. [11]), i.e. $\partial \gamma_{C}(x)=\left\{x^{*} \in C^{0}:\left\langle x^{*}, x\right\rangle=\sigma_{C^{0}}(x)\right\}$.

Remark 2.4 Results connected to the situation when the regularity condition $(b)$ in Theorem 2.1 is fulfilled can be found, for instance, in [23]. The support function of a compact convex set is real valued and continuous, however, in the absence of compactness it is surely continuous only on the (relative) interior of its domain, while an indicator function is continuous over the interior of the corresponding set.

Taking $f$ to be a gauge or an indicator function one obtains the following geometrical interpretations of the generalization of the extended perturbed minimal time function.

Remark 2.5 Let $C, \Omega, G \subseteq X$ be convex and closed sets such that $0_{X} \in C \cap G$. Then

$$
\begin{aligned}
\mathcal{T}_{\Omega, \gamma_{G}}^{-C}(x) & =\inf _{\substack{\alpha, \beta>0, z \in \Omega, y \in X, x-y-z \in-\alpha C, y \in \beta G}}\{\alpha+\beta\}=\inf _{\substack{\alpha, \beta>0, z \in \Omega, k \in X, x-k \in-\alpha C, k-z \in \beta G}}\{\alpha+\beta\} \\
= & \inf _{\substack{\alpha, \beta>0,(x+\alpha C) \cap(\Omega+\beta G) \neq \emptyset}}\{\alpha+\beta\} .
\end{aligned}
$$

The last formula suggests interpreting $\alpha$ as the minimal time needed for the given point $x$ to reach the set $\Omega$ along the constant dynamics $-C$, while $\Omega$ is moving in direction of $x$ with respect to the constant dynamics characterized by the set $G$. The value $\beta$ gives then the minimal time needed for $\Omega$ to reach $x$.

Remark 2.6 Let $S \subseteq X, \Omega$ and $C$ be convex and closed, with $S \neq \varnothing$ and $0_{X} \in C$. Then

$$
\begin{aligned}
\mathcal{T}_{\Omega, \delta_{S}}^{-C}(x) & =\inf \{\lambda>0: y \in S, z \in \Omega, x-y-z \in-\lambda C\} \\
& =\inf \{\lambda>0:(x+\lambda C) \cap(S+\Omega) \neq \emptyset\} .
\end{aligned}
$$

The extended perturbed minimal time function reduces to the classical minimal time function with the target set $S+\Omega$, i.e. if the set $C$ describes constant dynamics, then $\mathcal{T}_{\Omega, \delta_{S}}^{-C}(x)$ is the minimal time $\lambda>0$ needed for the point $x$ to reach the target set $S+\Omega$ (see for instance [13]). However, one can also write

$$
\begin{aligned}
\mathcal{T}_{\Omega, \delta_{S}}^{-C}(x) & =\inf \{\lambda>0: y \in S, z \in \Omega, x-y-z \in-\lambda C\} \\
& =\inf \{\lambda>0:(x+S+\lambda C) \cap \Omega \neq \emptyset\},
\end{aligned}
$$

and, when $C$ characterizes again constant dynamics, $\mathcal{T}_{\Omega, \delta_{S}}^{-C}(x)$ can be understood as the minimal time $\lambda>0$ needed for the set $S$ translated by the point $x$ to reach the target set $\Omega$.

Remark 2.7 When $C$ is convex and closed with $0 \in C, \Omega$ is convex and compact and $f=\delta_{\left\{0_{X}\right\}}$, then $\sigma_{\Omega}$ is continuous and $\mathcal{T}_{\Omega, \delta_{\left\{0_{X}\right\}}}^{C}$ is proper, convex and lower semicontinuous by Theorem 2.1, and can be written as $\mathcal{T}_{\Omega, \delta_{\left\{0_{X}\right\}}}^{C}=\min _{z \in \Omega} \gamma_{C}(\cdot-z)$. This statement can also be found in the special case $X=\mathbb{R}^{n}$ in [13, Theorem 3.33 and Theorem 4.7]. Moreover, in [12] it is assumed that $\gamma_{C} \square\left(f+\delta_{\Omega}\right)$ is exact, under similar hypotheses that would actually guarantee this outcome.

Remark 2.8 If $0_{X} \in \operatorname{core} C, \gamma_{C}$ has a full domain, consequently so does the corresponding extended perturbed minimal time function since in general $\operatorname{dom} \mathcal{T}_{\Omega, f}^{C}=\operatorname{dom} \gamma_{C}+\operatorname{dom} f+$ $\operatorname{dom} \delta_{\Omega}$. 


\section{Duality results}

\subsection{Location problem with perturbed minimal time functions}

In [27] the authors approached nonlinear minmax location problems by means of the conjugate duality in the case where the distances were measured by gauge functions. In this section we investigate such location problems in a more general setting, namely, where the distances are measured by perturbed minimal time functions.

Let $X$ be a Banach space (note that most of the following investigations can be extended to a Fréchet space, too) and $a_{i} \in \mathbb{R}_{+}, i=1, \ldots, n$, be given nonnegative set-up costs, where $n \geq 2$ and consider the following generalized location problem

$$
\left(P_{h, \mathcal{T}}^{S}\right) \inf _{x \in S} \max _{1 \leq i \leq n}\left\{h_{i}\left(\mathcal{T}_{\Omega_{i}, f_{i}}^{C_{i}}(x)\right)+a_{i}\right\},
$$

where $S \subseteq X$ is nonempty, closed and convex, $C_{i} \subseteq X$ is closed and convex with $0_{X} \in \operatorname{int} C_{i}$, $\Omega_{i} \subseteq X$ is nonempty, convex and compact, $f_{i}: X \rightarrow \overline{\mathbb{R}}$ is proper, convex and lower semicontinuous, $h_{i}: \mathbb{R} \rightarrow \overline{\mathbb{R}}$ with $h_{i}(x) \in \mathbb{R}_{+}$, if $x \in \mathbb{R}_{+}$, and $h_{i}(x)=+\infty$, otherwise, is proper, convex, lower semicontinuous and increasing on $\mathbb{R}_{+}, i=1, \ldots, n$.

Note that the assumptions made above yield that $0_{X^{*}} \in C_{i}^{0} \cap \operatorname{dom} \sigma_{\Omega} \cap \operatorname{dom} f_{i}^{*}$ and as $\gamma_{C_{i}}^{*}=\delta_{C^{0}}$ and $\sigma_{\Omega_{i}}$ are continuous functions (as $0 \in \operatorname{int} C_{i}$ and $\Omega_{i}$ is convex and compact), one gets by Theorem 2.1 that $\mathcal{T}_{\Omega_{i}, f_{i}}^{C_{i}}$ is a proper, convex and lower semicontinuous function with full domain and thus continuous, $i=1, \ldots, n$. Moreover, since $h_{i}$ is a proper, convex, lower semicontinuous and increasing function, $i=1, \ldots, n$, it follows that the objective function of $\left(P_{h, \mathcal{T}}^{S}\right)$ is proper, convex and lower semicontinuous, which means that $\left(P_{h, \mathcal{T}}^{S}\right)$ is a convex optimization problem.

Now, we analyze how can be understood the location problem $\left(P_{h, \mathcal{T}}^{S}\right)$ in the more simple situation where the function $h_{i}$ is linear continuous on $(0,+\infty)$ and $f_{i}$ is the indicator function of a nonempty, closed and convex subset of $X, i=1, \ldots, n$.

Remark 3.1 In the context of the Remark 2.5 let us consider the following concrete minmax location problem where $h_{i}(x)=x+\delta_{\mathbb{R}_{+}}(x), x \in \mathbb{R}, a_{i}=0$, and $C_{i}, G_{i}$ and $\Omega_{i}$ are closed and convex sets such that $0_{X} \in C_{i} \cap G_{i}$ for all $i=1, \ldots, n$,

$$
\begin{aligned}
& \left(P_{\gamma_{G}, \mathcal{T}}^{S}\right) \inf _{x \in X} \max _{1 \leq i \leq n}\left\{\mathcal{T}_{\Omega_{i}, \gamma_{G_{i}}}^{-C_{i}}(x)\right\}=\inf _{\substack{x \in X, t \in \mathbb{R}, \mathcal{T}_{\Omega_{i}, \gamma_{G_{i}}}^{-C_{i}} \\
i=1, \ldots, n}} t \\
& =\inf _{x \in X, t \in \mathbb{R}, \inf \left\{\alpha_{i}+\beta_{i}>0:\left(x+\alpha_{i} C_{i}\right) \cap\left(z_{i}+\beta_{i} G_{i}\right)\right\} \leq t,} t=\inf _{z_{i} \in \Omega_{i}, i=1, \ldots, n} t . \begin{array}{c}
x \in X, \alpha_{i}, \beta_{i}, t>0, \alpha_{i}+\beta_{i} \leq t, z_{i} \in \Omega_{i}, \\
\left(x+\alpha_{i} C_{i}\right) \cap\left(z_{i}+\beta_{i} G_{i}\right) \neq \emptyset, i=1, \ldots, n
\end{array},
\end{aligned}
$$

The last formulation allows the following economical interpretation. Given $n$ countries, each with a growing demand $G_{i}$ for a product and an average income (or average budget) characterized by the set $\Omega_{i}, i=1, \ldots, n$, consider a company, which produces and sells this product, planning to build a production facility. The production speed of the production facility as well as the preference of the company for a country are characterized by the sets $C_{i}$, $i=1, \ldots, n$. Then the objective of the company is to determine a location $\bar{x}$ for a production facility such that the total demand for the product can be satisfied in the shortest time, i.e. the company wants to enter all lucrative markets as fast as possible. Additionally, in the general case when the functions $h_{1}, \ldots, h_{n}$ are not necessarily linear (over their domains), these can be seen as cost or production functions, while the set-up costs $a_{1}, \ldots, a_{n}$ are the costs of the testing of the products to meet the various specifications asked by each of the $n$ countries. 
Remark 3.2 When $L_{i} \subseteq X, i=1, \ldots, n$, are nonempty, closed and convex sets, $f_{i}=\delta_{L_{i}}$ and $h_{i}=\cdot+\delta_{\mathbb{R}_{+}}(\cdot), i=1, \ldots, n,\left(P_{h, \mathcal{T}}^{S}\right)$ reads as (see also Remark 2.6)

$$
\left(P_{\mathcal{T}}^{S}\right) \inf _{x \in S} \max _{1 \leq i \leq n}\left\{\mathcal{T}_{\Omega_{i}, \delta_{L_{i}}}^{C_{i}}(x)+a_{i}\right\}=\inf _{\substack{x \in S, t \in \mathbb{R}, \mathcal{T}_{\Omega_{i}, \delta_{L_{i}}}^{C_{i}}(x)+a_{i} \leq t, i=1, \ldots, n}} t=\inf _{\substack{x \in S, t \in \mathbb{R}, \inf \left\{\lambda_{i}>0:\left(x-\lambda_{i} C_{i}\right) \cap\left(\Omega_{i}+L_{i}\right) \neq \emptyset\right\}+a_{i} \leq t, i=1, \ldots, n}} t
$$

and can be seen as finding a point $x \in S$ and the smallest number $t>0$ such that

$$
\left(x-\left(t-a_{i}\right) C_{i}\right) \cap\left(\Omega_{i}+L_{i}\right) \neq \emptyset \quad \forall i=1, \ldots, n,
$$

where $C_{i}$ can be defined as a generalized ball with radius $t-a_{i}, i=1, \ldots, n$ (see [13]). This approach is especially useful if the target set is hard to handle, but can be split into a Minkowski sum of two simpler sets $\Omega_{i}$ and $L_{i}, i=1, \ldots, n$, as happens for instance with the rounded rectangles that can be written as sums of rectangles and circles. Note also that [2] addresses the situation when the projection onto a Minkowski sum of closed convex sets coincides with the sums of projections into these sets. Alternatively, $\left(P_{\mathcal{T}}^{S}\right)$ can be written as

$$
\left(P_{\mathcal{T}}^{S}\right) \inf _{x \in S} \max _{1 \leq i \leq n}\left\{\mathcal{T}_{\Omega_{i}, \delta_{L_{i}}}^{C_{i}}(x)+a_{i}\right\}=\inf _{\substack{x \in S, t \in \mathbb{R}, \inf \left\{\lambda_{i}>0:\left(x-L_{i}-\lambda_{i} C_{i}\right) \cap \Omega_{i} \neq \emptyset\right\}+a_{i} \leq t, i=1, \ldots, n}} t,
$$

which allows the interpretation as finding a point $x \in S$ and the smallest number $t>0$ such that

$$
\left(x-L_{i}-\left(t-a_{i}\right) C_{i}\right) \cap \Omega_{i} \neq \emptyset \quad \forall i=1, \ldots, n .
$$

Both (2) and (3) are generalizations of the classical Sylvester problem that consists in finding the smallest circle that encloses finitely many given points.

In order to approach the problem $\left(P_{h, \mathcal{T}}^{S}\right)$ by means of the conjugate duality concept introduced in [25] (with $X_{0}=\mathbb{R}^{n}$ partially ordered by the convex cone $K_{0}=\mathbb{R}_{+}^{n}, X_{1}=X^{n}$ partially ordered by the trivial cone $K_{1}=\left\{0_{X^{n}}\right\}$ and $X_{2}=X$ ), we consider the following functions

$$
f: \mathbb{R}^{n} \rightarrow \overline{\mathbb{R}}, f(z):= \begin{cases}\max _{1 \leq i \leq n}\left\{h_{i}\left(z_{i}\right)+a_{i}\right\}, & \text { if } z=\left(z_{1}, \ldots, z_{n}\right)^{\top} \in \mathbb{R}_{+}^{n}, i=1, \ldots, n, \\ +\infty, & \text { otherwise, }\end{cases}
$$

$F: X^{n} \rightarrow \mathbb{R}^{n}, F\left(y_{1}, \ldots, y_{n}\right):=\left(\mathcal{T}_{\Omega_{1}, f_{1}}^{C_{1}}\left(y_{1}\right), \ldots, \mathcal{T}_{\Omega_{n}, f_{n}}^{C_{n}}\left(y_{n}\right)\right)^{\top}$ and $G: X \rightarrow X^{n}$, $G(x):=(x, \ldots, x)$. With these newly introduced functions we can write the optimization problem $\left(P_{h, \mathcal{T}}^{S}\right)$ as a multi-composed optimization problem (cf. [25,27])

$$
\left(P_{h, \mathcal{T}}^{S}\right) \inf _{x \in S}(f \circ F \circ G)(x) .
$$

Notice that the function $f$ is proper, convex, $\mathbb{R}_{+}^{n}$-increasing on $F(\operatorname{dom} F)+K_{0}=$ $\operatorname{dom} f=\mathbb{R}_{+}^{n}$ and lower semicontinous. Moreover, as the functions $\mathcal{T}_{\Omega_{i}, f_{i}}^{C_{i}}, i=1, \ldots, n$, are proper, convex and lower semicontinuous, it is obvious that the function $F$ is proper, $\mathbb{R}_{+}^{n}$ convex and $\mathbb{R}_{+}^{n}$-epi-closed. In addition, as the function $G$ is linear continuous, it follows that the function $F$ does not need to be monotone as asked in the general theory in $[10,25,27,29]$. 
Employing the duality concept introduced in $[10,25]$ we attach to $\left(P_{h, \mathcal{T}}^{S}\right)$ the following conjugate dual problem

$$
\left(D_{h, \mathcal{T}}^{S}\right) \quad \sup _{\substack{z_{i}^{*} \in \mathbb{R}_{+}, w_{i}^{*} \in X^{*}, i=1, \ldots, n}}\left\{\inf _{x \in S}\left\{\sum_{i=1}^{n}\left\langle w_{i}^{*}, x\right\rangle\right\}-f^{*}\left(z^{*}\right)-\left(z^{*} F\right)^{*}\left(w^{*}\right)\right\},
$$

where $z^{*}=\left(z_{1}^{*}, \ldots, z_{n}^{*}\right)^{\top} \in \mathbb{R}_{+}^{n}$ and $w^{*}=\left(w_{1}^{*}, \ldots, w_{n}^{*}\right) \in\left(X^{*}\right)^{n}$ are the dual variables. By [27, Theorem 4] one has

$$
f^{*}\left(z_{1}^{*}, \ldots, z_{n}^{*}\right)=\min _{\substack{i=1 \\ \sum_{i=1} \lambda_{i} \leq 1, \lambda_{i} \geq 0, i=1, \ldots, n}}\left\{\sum_{i=1}^{n}\left[\left(\lambda_{i} h_{i}\right)^{*}\left(z_{i}^{*}\right)-\lambda_{i} a_{i}\right]\right\},
$$

while $\left(z^{*} F\right)^{*}\left(w^{*}\right)=\sum_{i=1}^{n}\left(z_{i}^{*} \mathcal{T}_{\Omega_{i}, f_{i}}^{C_{i}}\right)^{*}\left(w_{i}^{*}\right)$, thus $\left(D_{h, \mathcal{T}}^{S}\right)$ becomes

$$
\begin{aligned}
&\left(D_{h, \mathcal{T}}^{S}\right) \sup _{\substack{\sum_{i=1}^{n} \lambda_{i} \leq 1, \lambda_{i}, z_{i}^{*} \geq 0, w_{i}^{*} \in X^{*}, i=1, \ldots, n}}\left\{-\sigma_{S}\left(-\sum_{i=1}^{n} w_{i}^{*}\right)-\sum_{i=1}^{n}\left[\left(\lambda_{i} h_{i}\right)^{*}\left(z_{i}^{*}\right)-\lambda_{i} a_{i}\right]\right. \\
&\left.-\sum_{i=1}^{n}\left(z_{i}^{*} \mathcal{T}_{\Omega_{i}, f_{i}}^{C_{i}}\right)^{*}\left(w_{i}^{*}\right)\right\} .
\end{aligned}
$$

In order to investigate further this dual problem, we separate in the sum $\sum_{i=1}^{n}\left(\lambda_{i} h_{i}\right)^{*}$ the terms with $\lambda_{i}>0$ and the terms with $\lambda_{i}=0$ as well as in $\sum_{i=1}^{n}\left(z_{i}^{*} \mathcal{T}_{\Omega_{i}, f_{i}}^{C_{i}}\right)^{*}$ the terms with $z_{i}^{*}>0$ and the terms with $z_{i}^{*}=0$ in $\left(D_{h, \mathcal{T}}^{S}\right)$. Denote $I=\left\{i \in\{1, \ldots, n\}: z_{i}^{*}>0\right\}$ and $R=\left\{r \in\{1, \ldots, n\}: \lambda_{r}>0\right\}$. If $i \in\{1, \ldots, n\} \backslash I$ it holds $\left(0 \cdot \mathcal{T}_{\Omega_{i}, f_{i}}^{C_{i}}\right)^{*}=\sigma_{X}=\delta_{\left\{0_{X^{*}}\right\}}$, while when $i \in I$ one gets

$$
\left(z_{i}^{*} \mathcal{T}_{\Omega_{i}, f_{i}}^{C_{i}}\right)^{*}\left(w_{i}^{*}\right)= \begin{cases}z_{i}^{*} f_{i}^{*}\left(\frac{1}{z_{i}^{*}} w_{i}^{*}\right)+\sigma_{\Omega_{i}}\left(w_{i}^{*}\right), & \text { if } \gamma_{C_{i}^{0}}\left(w_{i}^{*}\right) \leq z_{i}^{*}, \\ +\infty, & \text { otherwise. }\end{cases}
$$

Further, let us consider the case $r \in\{1, \ldots, n\} \backslash R$, i.e. $\lambda_{r}=0$, then one has, since $z_{r}^{*} \geq 0$,

$$
\left(0 \cdot h_{r}\right)^{*}\left(z_{r}^{*}\right)=\sup _{z_{r} \geq 0}\left\{z_{r}^{*} z_{r}\right\}= \begin{cases}0, & \text { if } z_{r}^{*}=0, \\ +\infty, & \text { otherwise. }\end{cases}
$$

For $r \in R$, i.e. $\lambda_{r}>0$, follows

$$
\left(\lambda_{r} h_{r}\right)^{*}\left(z_{r}^{*}\right)=\lambda_{r} h_{r}^{*}\left(\frac{z_{r}^{*}}{\lambda_{r}}\right) .
$$

Hence, formula (5) implies that if $r \notin R$ then $z_{r}^{*}=0$, otherwise the values being not relevant for the dual problem, which means that $I \subseteq R$. Therefore $\left(D_{h, \mathcal{T}}^{S}\right)$ turns into

$\sup$

$\lambda_{i}, z_{i}^{*} \geq 0, i=1, \ldots, n$,

$I=\left\{i \in\{1, \ldots, n\}: z_{i}^{*}>0\right\} \subseteq R=\left\{r \in\{1, \ldots, n\}: \lambda_{r}>0\right\}$,

$$
\left\{-\sigma_{S}\left(-\sum_{i \in I} w_{i}^{*}\right)-\sum_{r \in R} \lambda_{r}\left[h_{r}^{*}\left(\frac{z_{r}^{*}}{\lambda_{r}}\right)-a_{r}\right]\right.
$$

$w_{i}^{*} \in X^{*}, \gamma_{C_{i}^{0}}\left(w_{i}^{*}\right) \leq z_{i}^{*}, i \in I, \quad \sum_{r \in R} \lambda_{r} \leq 1$ 


$$
\left.-\sum_{i \in I}\left[z_{i}^{*} f_{i}^{*}\left(\frac{1}{z_{i}^{*}} w_{i}^{*}\right)+\sigma_{\Omega_{i}}\left(w_{i}^{*}\right)\right]\right\} .
$$

Remark 3.3 Let $a_{i}=0, i=1, \ldots, n$. Taking the functions $h_{i}$ as in Remark 3.2, their conjugates are $h_{i}^{*}=\delta_{(-\infty, 1]}, i=1, \ldots, n$, and the conjugate dual problem to $\left(P_{\mathcal{T}}^{S}\right)$ reads as

$$
\begin{gathered}
\left(D_{\mathcal{T}}^{S}\right) \sup _{\substack{\lambda_{i}, z_{i}^{*} \geq 0, i=1, \ldots, n, \sum_{r \in R} \lambda_{r} \leq 1, I=\left\{i \in\{1, \ldots, n\}: z_{i}^{*}>0\right\} \subseteq R=\left\{r \in\{1, \ldots, n\}: \lambda_{r}>0\right\}, z_{r}^{*} \leq \lambda_{r}, r \in R, w_{i}^{*} \in X^{*}, \gamma_{C_{i}^{0}}\left(w_{i}^{*}\right) \leq z_{i}^{*}, i \in I}}\left\{-\sigma_{S}\left(-\sum_{i \in I} w_{i}^{*}\right)\right. \\
\left.-\sum_{i \in I}\left[z_{i}^{*} f_{i}^{*}\left(\frac{1}{z_{i}^{*}} w_{i}^{*}\right)+\sigma_{\Omega_{i}}\left(w_{i}^{*}\right)\right]\right\} .
\end{gathered}
$$

This dual problem can be simplified as follows.

Proposition 3.1 The problem $\left(D_{\mathcal{T}}^{S}\right)$ can be equivalently written as

$$
\begin{aligned}
& \left(\widetilde{D}_{\mathcal{T}}^{S}\right)_{\substack{u_{i}^{*} \geq 0, i=1, \ldots, n, \widetilde{I}=\left\{i \in\{1, \ldots, n\}: u_{i}^{*}>0\right\}, v_{i}^{*} \in X^{*}, i \in \widetilde{I}, \gamma_{C_{i}^{0}}\left(v_{i}^{*}\right) \leq u_{i}^{*}, i \in \widetilde{I}, \sum_{i \in \widetilde{I}} u_{i}^{*} \leq 1}}\left\{-\sigma_{S}\left(-\sum_{i \in \widetilde{I}} v_{i}^{*}\right)\right. \\
& \left.-\sum_{i \in \widetilde{I}}\left[u_{i}^{*} f_{i}^{*}\left(\frac{1}{u_{i}^{*}} v_{i}^{*}\right)+\sigma_{\Omega_{i}}\left(v_{i}^{*}\right)\right]\right\} .
\end{aligned}
$$

Proof Take first a feasible element $\left(\lambda, z^{*}, w^{*}\right)=\left(\lambda_{1}, \ldots, \lambda_{n}, z_{1}^{*}, \ldots, z_{n}^{*}, w_{I}^{*}\right) \in \mathbb{R}_{+}^{n} \times$ $\mathbb{R}_{+}^{n} \times\left(X^{*}\right)^{|I|}$ to the problem $\left(D_{\mathcal{T}}^{S}\right)$, where by $w_{I}^{*} \in\left(X^{*}\right)^{|I|}$ we denote the vector having as components $w_{i}^{*}$ with $i \in I$, and set $\widetilde{I}=I, u_{i}^{*}=\lambda_{i}, i \in \widetilde{I}, u_{j}^{*}=0, j \notin \widetilde{I}$ and $v_{i}^{*}=w_{i}^{*}, i \in$ $\widetilde{I}, v_{j}^{*}=0_{X^{*}}, j \notin \widetilde{I}$, then it follows from the feasibility of $\left(\lambda, z^{*}, w^{*}\right)$ that $\sum_{i \in \widetilde{I}}^{n} u_{i}^{*} \leq$ $1, u_{i}^{*}>0, v_{i}^{*} \in X^{*}, \gamma_{C_{i}^{0}}\left(v_{i}^{*}\right) \leq u_{i}^{*}, i \in \widetilde{I}$ and $u_{j}^{*}=0, j \notin \widetilde{I}$, i.e. $\left(u^{*}, v^{*}\right) \in \mathbb{R}_{+}^{n} \times\left(X^{*}\right)^{|\widetilde{I}|}$ is feasible to the problem $\left(\widetilde{D}_{\mathcal{T}}^{S}\right)$. Hence, it holds $-\sigma_{S}\left(-\sum_{i \in I} w_{i}^{*}\right)-\sum_{i \in I}\left[z_{i}^{*} f_{i}^{*}\left(\left(1 / z_{i}^{*}\right) w_{i}^{*}\right)+\right.$ $\left.\sigma_{\Omega_{i}}\left(w_{i}^{*}\right)\right]=-\sigma_{S}\left(-\sum_{i \in \tilde{I}} v_{i}^{*}\right)-\sum_{i \in \tilde{I}}\left[u_{i}^{*} f_{i}^{*}\left(\left(1 / u_{i}^{*}\right) v_{i}^{*}\right)+\sigma_{\Omega_{i}}\left(v_{i}^{*}\right)\right] \leq v\left(\widetilde{D}_{\mathcal{T}}^{S}\right)$ for all $\left(\lambda, z^{*}, w^{*}\right)$ feasible to $\left(D_{\mathcal{T}}^{S}\right)$, i.e. $v\left(D_{\mathcal{T}}^{S}\right) \leq v\left(\widetilde{D}_{\mathcal{T}}^{S}\right)$.

To prove the opposite inequality, take a feasible element $\left(u^{*}, v^{*}\right)$ of the problem $\left(\widetilde{D}_{\mathcal{T}}^{S}\right)$ and set $I=R=\widetilde{I}, z_{i}^{*}=\lambda_{i}=u_{i}^{*}$ and $w_{i}^{*}=v_{i}^{*}$ for $i \in I=R$ and $z_{j}^{*}=\lambda_{j}=0$ for $j \notin I=R$, then we have from the feasibility of $\left(u^{*}, v^{*}\right)$ that $\sum_{r \in R} \lambda_{r} \leq 1, z_{k}^{*}=\lambda_{k}>$ $0, k \in R, \lambda_{l}=0, l \notin R$ and $\gamma_{C_{i}^{0}}\left(w_{i}^{*}\right) \leq z_{i}^{*}, i \in I$, which means that $\left(\lambda, z^{*}, w^{*}\right)$ is a feasible element of $\left(D_{\mathcal{T}}^{S}\right)$ and it holds $-\sigma_{S}\left(-\sum_{i \in I} v_{i}^{*}\right)-\sum_{i \in I}^{n}\left[u_{i}^{*} f_{i}^{*}\left(\left(1 / u_{i}^{*}\right) v_{i}^{*}\right)+\sigma_{\Omega_{i}}\left(v_{i}^{*}\right)\right]=$ $-\sigma_{S}\left(-\sum_{i \in I} w_{i}^{*}\right)-\sum_{i \in I}\left[z_{i}^{*} f_{i}^{*}\left(\left(1 / z_{i}^{*}\right) w_{i}^{*}\right)+\sigma_{\Omega_{i}}\left(w_{i}^{*}\right)\right] \leq v\left(D_{\mathcal{T}}^{S}\right)$ for all $\left(u^{*}, v^{*}\right)$ feasible to $\left(\widetilde{D}_{\mathcal{T}}^{S}\right)$, which implies $v\left(\widetilde{D}_{\mathcal{T}}^{S}\right) \leq v\left(D_{\mathcal{T}}^{S}\right)$. Finally, it follows that $v\left(\widetilde{D}_{\mathcal{T}}^{S}\right)=v\left(D_{\mathcal{T}}^{S}\right)$.

Also the general dual problem $\left(D_{h, \mathcal{T}}^{S}\right)$ can be rewritten as follows. 
Proposition 3.2 The problem $\left(D_{h, \mathcal{T}}^{S}\right)$ can be equivalently written as

$$
\begin{aligned}
&\left(\widehat{D}_{h, \mathcal{T}}^{S}\right) \sup _{\substack{\lambda_{i}, z_{i}^{*} \geq 0, w_{i}^{*} \in X^{*}, \sum_{i=1}^{n} \lambda_{i} \leq 1, \gamma_{C_{i}^{0}\left(w_{i}^{*}\right) \leq z_{i}^{*}, i=1, \ldots, n}}}\left\{-\sigma_{S}\left(-\sum_{i=1}^{n} w_{i}^{*}\right)-\sum_{i=1}^{n}\left[\left(\lambda_{i} h_{i}\right)^{*}\left(z_{i}^{*}\right)-\lambda_{i} a_{i}\right.\right. \\
&\left.\left.+\left(z_{i}^{*} f_{i}\right)^{*}\left(w_{i}^{*}\right)+\sigma_{\Omega_{i}}\left(w_{i}^{*}\right)\right]\right\} .
\end{aligned}
$$

Proof Let $\left(\lambda_{1}, \ldots, \lambda_{n}, z_{1}^{*}, \ldots, z_{n}^{*}, w_{1}^{*}, \ldots, w_{n}^{*}\right)$ be a feasible solution to $\left(\widehat{D}_{h, \mathcal{T}}^{S}\right)$, then it follows from $r \notin R=\left\{r \in\{1, \ldots, n\}: \lambda_{r}>0\right\}$ by (5) that $z_{r}^{*}=0$, i.e. $I=$ $\left\{i \in\{1, \ldots, n\}: z_{i}^{*}>0\right\} \subseteq R$, and for $i \in\{1, \ldots, n\} \backslash I$ we have $0 \leq \gamma_{C_{i}^{0}}\left(w_{i}^{*}\right) \leq 0 \Leftrightarrow$ $w_{i}^{*}=0_{X^{*}}$. This means that $\left(\lambda_{1}, \ldots, \lambda_{n}, z_{1}^{*}, \ldots, z_{n}^{*}, w_{I}^{*}\right)$ is feasible to $\left(D_{h, \mathcal{T}}^{S}\right)$ and by (5) and (6) follows immediately that $v\left(\widehat{D}_{h, \mathcal{T}}^{S}\right) \leq v\left(D_{h, \mathcal{T}}^{S}\right)$.

Conversely, by the previous considerations it is clear that from any feasible solution to $\left(D_{h, \mathcal{T}}^{S}\right)$ one can immediately construct a feasible solution to $\left(\widehat{D}_{h, \mathcal{T}}^{S}\right)$ such that $v\left(D_{h, \mathcal{T}}^{S}\right) \leq$ $v\left(\widehat{D}_{h, \mathcal{T}}^{S}\right)$ by taking $w_{i}^{*}=0_{X^{*}}$ for $i \in\{1, \ldots, n\} \backslash I$.

Remark 3.4 The index sets $I$ and $R$ of the dual problem $\left(D_{h, \mathcal{T}}^{S}\right)$ in (7) give a minute characterization of the set of feasible solutions and are useful in the further approach. From the numerical aspect however, they transform the dual (7) into a discrete optimization problem, making it very hard to solve. For this reason we use for theoretical approaches the dual $\left(D_{h, \mathcal{T}}^{S}\right)$ in the form of (7) and for numerical studies its equivalent dual formulation provided in Proposition 3.2. In this context, the dual $\left(\widetilde{D}_{\mathcal{T}}^{S}\right)$ is equivalent to

$$
\begin{aligned}
& \left(\widetilde{D}_{\mathcal{T}}^{S}\right) \sup _{z_{i}^{*} \geq 0, w_{i}^{*} \in X^{*}, \gamma_{C_{i}^{0}}\left(w_{i}^{*}\right) \leq z_{i}^{*}}\left\{-\sigma_{S}\left(-\sum_{i=1}^{n} w_{i}^{*}\right)+\sum_{i=1}^{n}\left[z_{i}^{*} a_{i}-f_{i}^{*}\left(w_{i}^{*}\right)-\sigma_{\Omega_{i}}\left(w_{i}^{*}\right)\right]\right\} . \\
& i=1, \ldots, n, \sum_{i=1}^{n} z_{i}^{*} \leq 1
\end{aligned}
$$

The weak duality for the primal-dual pair $\left(P_{h, \mathcal{T}}^{S}\right)-\left(D_{h, \mathcal{T}}^{S}\right)$ holds by construction, i.e. $v\left(P_{h, \mathcal{T}}^{S}\right) \geq v\left(D_{h, \mathcal{T}}^{S}\right)$, and we show that the considered hypotheses guarantee strong duality, too.

Theorem 3.1 (Strong duality) Between $\left(P_{h, \mathcal{T}}^{S}\right)$ and $\left(D_{h, \mathcal{T}}^{S}\right)$ strong duality holds, i.e. $v\left(P_{h, \mathcal{T}}^{S}\right)=v\left(D_{h, \mathcal{T}}^{S}\right)$ and the conjugate dual problem has an optimal solution $\left(\bar{\lambda}_{1}, \ldots, \bar{\lambda}_{n}, \bar{z}_{1}^{*}\right.$, $\left.\ldots, \bar{z}_{n}^{*}, \bar{w}_{\bar{I}}^{*}\right) \in \mathbb{R}_{+}^{n} \times \mathbb{R}_{+}^{n} \times\left(X^{*}\right)^{|\bar{I}|}$ with the corresponding index sets $\bar{I} \subseteq \bar{R} \subseteq\{1, \ldots, n\}$.

Proof The conclusion follows by [25, Theorem 4], whose hypotheses are fulfilled as seen below. The properness and convexity properties of the involved functions and sets are guaranteed by the standing assumptions formulated in the beginning of the section. It remains to verify the fulfillment of a regularity condition. We use the generalized interior point regularity condition $\left(R C_{2}^{C}\right)$ introduced in [25] for multi-composed optimization problems that is a development of the one given in the general case in [30]. First, notice that $f$ is lower semicontinuous, $K_{0}=\mathbb{R}_{+}^{n}$ is closed and has a nonempty interior, $S$ is closed, $F$ is $\mathbb{R}_{+}^{n}$-epiclosed, while the linear continuous function $G$ is obviously $\left\{0_{X^{n}}\right\}$-epi-closed. The continuity of $G$ voids (see [25, Remark 5]) the necessity of having int $K_{1} \neq \emptyset$, a condition that is 
in this case not fulfilled. The other requirements of the regularity condition are fulfilled as well, namely $0_{X} \in \operatorname{sqri}((X \cap S)+X)=X, 0_{\mathbb{R}^{n}} \in \operatorname{sqri}\left(F(\operatorname{dom} F)-\operatorname{dom} f+K_{0}\right)=$ $\operatorname{sqri}\left(F(\operatorname{dom} F)-\mathbb{R}_{+}^{n}+\mathbb{R}_{+}^{n}\right)=\mathbb{R}^{n}$ and (recall that $\left.\operatorname{dom} \mathcal{T}_{\Omega_{i}, f_{i}}^{C_{i}}=X, i=1, \ldots, n\right)$ $0_{X^{n}} \in \operatorname{sqri}\left(G(\operatorname{dom} G \cap \operatorname{dom} g \cap S)-\operatorname{dom} F+K_{1}\right)=\operatorname{sqri}\left(G(S)-X^{n}+\left\{0_{X^{n}}\right\}\right)=X^{n}$.

The next statement is dedicated to deriving necessary and sufficient optimality conditions for the primal-dual pair $\left(P_{h, \mathcal{T}}^{S}\right)-\left(D_{h, \mathcal{T}}^{S}\right)$.

Theorem 3.2 (Optimality conditions) (a) Let $\bar{x} \in S$ be an optimal solution to the problem $\left(P_{h, \mathcal{T}}^{S}\right)$. Then there exists $\left(\bar{\lambda}_{1}, \ldots, \bar{\lambda}_{n}, \bar{z}_{1}^{*}, \ldots, \bar{z}_{n}^{*}, \bar{w}_{\bar{I}}^{*}\right) \in \mathbb{R}_{+}^{n} \times \mathbb{R}_{+}^{n} \times\left(X^{*}\right)^{|\bar{I}|}$ with the corresponding index sets $\bar{I} \subseteq \bar{R} \subseteq\{1, \ldots, n\}$ as an optimal solution to $\left(D_{h, \mathcal{T}}^{S}\right)$ such that

(i) $\max _{1 \leq j \leq n}\left\{h_{j}\left(\mathcal{T}_{\Omega_{j}, f_{j}}^{C_{j}}(\bar{x})\right)+a_{j}\right\}$

$=\sum_{i \in \bar{I}} \bar{z}_{i}^{*} \mathcal{T}_{\Omega_{i}, f_{i}}^{C_{i}}(\bar{x})-\sum_{r \in \bar{R}} \bar{\lambda}_{r}\left[h_{r}^{*}\left(\frac{\bar{z}_{r}^{*}}{\bar{\lambda}_{r}}\right)-a_{r}\right]=\sum_{r \in \bar{R}} \bar{\lambda}_{r}\left[h_{r}\left(\mathcal{T}_{\Omega_{r}, f_{r}}^{C_{r}}(\bar{x})\right)+a_{r}\right]$,

(ii) $\bar{\lambda}_{r} h_{r}^{*}\left(\frac{\bar{z}_{r}^{*}}{\bar{\lambda}_{r}}\right)+\bar{\lambda}_{r} h_{r}\left(\mathcal{T}_{\Omega_{r}, f_{r}}^{C_{r}}(\bar{x})\right)=\bar{z}_{r}^{*} \mathcal{T}_{\Omega_{r}, f_{r}}^{C_{r}}(\bar{x}) \forall r \in \bar{R}$,

(iii) $\bar{z}_{i}^{*} \mathcal{T}_{\Omega_{i}, f_{i}}^{C_{i}}(\bar{x})+\bar{z}_{i}^{*} f_{i}^{*}\left(\frac{1}{\bar{z}_{i}^{*}} \bar{w}_{i}^{*}\right)+\sigma_{\Omega_{i}}\left(\bar{w}_{i}^{*}\right)=\left\langle\bar{w}_{i}^{*}, \bar{x}\right\rangle \forall i \in \bar{I}$,

(iv) $\sum_{i \in \bar{I}}\left\langle\bar{w}_{i}^{*}, \bar{x}\right\rangle=-\sigma_{S}\left(-\sum_{i \in \bar{I}} \bar{w}_{i}^{*}\right)$,

(v) $\max _{1 \leq j \leq n}\left\{h_{j}\left(\mathcal{T}_{\Omega_{j}, f_{j}}^{C_{j}}(\bar{x})\right)+a_{j}\right\}=h_{r}\left(\mathcal{T}_{\Omega_{r}, f_{r}}^{C_{r}}(\bar{x})\right)+a_{r} \forall r \in \bar{R}$,

(vi) $\sum_{r \in \bar{R}} \bar{\lambda}_{r}=1, \bar{\lambda}_{k}>0, k \in \bar{R}, \bar{\lambda}_{l}=0, l \notin \bar{R}, \bar{z}_{i}^{*}>0, i \in \bar{I}$, and $\bar{z}_{j}^{*}=0, j \notin \bar{I}$,

(vii) $\gamma_{C_{i}^{0}}\left(\bar{w}_{i}^{*}\right)=\bar{z}_{i}^{*}, \bar{w}_{i}^{*} \in X^{*} \backslash\left\{0_{X^{*}}\right\}, i \in \bar{I}$.

(b) If there exists $\bar{x} \in S$ such that for some $\left(\bar{\lambda}_{1}, \ldots, \bar{\lambda}_{n}, \bar{z}_{1}^{*}, \ldots, \bar{z}_{n}^{*}, \bar{w}_{1}^{*}, \ldots, \bar{w}_{\bar{I}}^{*}\right) \in \mathbb{R}_{+}^{n} \times$ $\mathbb{R}_{+}^{n} \times\left(X^{*}\right)^{|\overline{\mid}|}$ with the corresponding index sets $\bar{I} \subseteq \bar{R} \subseteq\{1, \ldots, n\}$ the conditions $(i)-$ (vii) are fulfilled, then $\bar{x}$ is an optimal solution to $\left(P_{h, \mathcal{T}}^{S}\right),\left(\bar{\lambda}_{1}, \ldots, \bar{\lambda}_{n}, \bar{z}_{1}^{*}, \ldots, \bar{z}_{n}^{*}, \bar{w}_{\bar{I}}^{*}\right)$ is an optimal solution to $\left(D_{h, \mathcal{T}}^{S}\right)$ and $v\left(P_{h, \mathcal{T}}^{S}\right)=v\left(D_{h, \mathcal{T}}^{S}\right)$.

Proof (a) By [25, Theorem 5] we obtain the following necessary and sufficient optimality conditions for the primal-dual pair $\left(P_{h, \mathcal{T}}^{S}\right)-\left(D_{h, \mathcal{T}}^{S}\right)$

(i') $\max _{1 \leq j \leq n}\left\{h_{j}\left(\mathcal{T}_{\Omega_{j}, f_{j}}^{C_{j}}(\bar{x})\right)+a_{j}\right\}+\sum_{r \in \bar{R}} \bar{\lambda}_{r}\left[h_{r}^{*}\left(\frac{\bar{z}_{r}^{*}}{\bar{\lambda}_{r}}\right)-a_{r}\right]=\sum_{i \in \bar{I}} \bar{z}_{i}^{*} \mathcal{T}_{\Omega_{i}, f_{i}}^{C_{i}}(\bar{x})$,

(ii') $\sum_{i \in \bar{I}} \bar{z}_{i}^{*} \mathcal{T}_{\Omega_{i}, f_{i}}^{C_{i}}(\bar{x})+\sum_{i \in \bar{I}}\left[\bar{z}_{i}^{*} f_{i}^{*}\left(\frac{1}{\bar{z}_{i}^{*}} \bar{w}_{i}^{*}\right)+\sigma_{\Omega_{i}}\left(\bar{w}_{i}^{*}\right)\right]=\sum_{i \in \bar{I}}\left\langle\bar{w}_{i}^{*}, \bar{x}\right\rangle$,

(iii') $\sum_{i \in \bar{I}}\left\langle\bar{w}_{i}^{*}, \bar{x}\right\rangle+\sigma_{S}\left(-\sum_{i \in \bar{I}} \bar{w}_{i}^{*}\right)=0$,

(iv') $\sum_{r \in \bar{R}} \bar{\lambda}_{r} \leq 1, \bar{\lambda}_{k}>0, k \in \bar{R}, \bar{\lambda}_{l}=0, l \notin \bar{R}, \bar{z}_{i}^{*}>0, i \in \bar{I}$, and $\bar{z}_{j}^{*}=0, j \notin \bar{I}$,

(v') $\gamma_{C_{i}^{0}}\left(\bar{w}_{i}^{*}\right) \leq \bar{z}_{i}^{*}, \bar{w}_{i}^{*} \in X^{*}, i \in \bar{I}$.

Additionally, one has by Theorem 3.1 that $v\left(P_{h, a}^{S}\right)=v\left(D_{h, a}^{S}\right)$, i.e.

$$
\max _{1 \leq j \leq n}\left\{h_{j}\left(\mathcal{T}_{\Omega_{j}, f_{j}}^{C_{j}}(\bar{x})\right)+a_{j}\right\}=-\sigma_{S}\left(-\sum_{i \in \bar{I}} \bar{w}_{i}^{*}\right)-\sum_{r \in \bar{R}} \bar{\lambda}_{r}\left[h_{r}^{*}\left(\frac{\bar{z}_{r}^{*}}{\bar{\lambda}_{r}}\right)-a_{r}\right]
$$




$$
-\sum_{i \in \bar{I}}\left[\bar{z}_{i}^{*} f_{i}^{*}\left(\frac{1}{\bar{z}_{i}^{*}} \bar{w}_{i}^{*}\right)+\sigma_{\Omega_{i}}\left(\bar{w}_{i}^{*}\right)\right]
$$

that can be equivalently written as

$$
\begin{aligned}
& {\left[\max _{1 \leq j \leq n}\left\{h_{j}\left(\mathcal{T}_{\Omega_{j}, f_{j}}^{C_{j}}(\bar{x})\right)+a_{j}\right\}-\sum_{r \in \bar{R}}\left(\bar{\lambda}_{r} h_{r}\left(\mathcal{T}_{\Omega_{r}, f_{r}}^{C_{r}}(\bar{x})\right)+\bar{\lambda}_{r} a_{r}\right)\right]} \\
& +\sum_{i \in \bar{I}}\left[\bar{z}_{i}^{*}\left(\mathcal{T}_{\Omega_{i}, f_{i}}^{C_{i}}(\bar{x})\right)+\bar{z}_{i}^{*} f_{i}^{*}\left(\frac{1}{\bar{z}_{i}^{*}} \bar{w}_{i}^{*}\right)+\sigma_{\Omega_{i}}\left(\bar{w}_{i}^{*}\right)-\left\langle\bar{w}_{i}^{*}, \bar{x}\right\rangle\right] \\
& \left.+\sigma_{S}\left(-\sum_{i \in \bar{I}} \bar{w}_{i}^{*}\right)+\sum_{i \in \bar{I}}\left\langle\bar{w}_{i}^{*}, \bar{x}\right\rangle\right]+\sum_{i \in \bar{I}}\left[\bar{\lambda}_{i} h_{i}^{*}\left(\frac{\bar{z}_{i}^{*}}{\bar{\lambda}_{i}}\right)\right. \\
& \left.+\bar{\lambda}_{i} h_{i}\left(\mathcal{T}_{\Omega_{i}, f_{i}}^{C_{i}}(\bar{x})\right)-\bar{z}_{i}^{*} \mathcal{T}_{\Omega_{i}, f_{i}}^{C_{i}}(\bar{x})\right] \\
& +\sum_{r \in \bar{R} \backslash \bar{I}}\left[\bar{\lambda}_{r} h_{r}^{*}(0)+\bar{\lambda}_{r} h_{r}\left(\mathcal{T}_{\Omega_{r}, f_{r}}^{C_{r}}(\bar{x})\right)-0 \cdot\left(\mathcal{T}_{\Omega_{r}, f_{r}}^{C_{r}}(\bar{x})\right)\right]=0,
\end{aligned}
$$

where the last two sums arise from the fact that $\bar{I} \subseteq \bar{R}$. By [27, Lemma 2] holds that the term within the first pair of brackets above is nonnegative. Moreover, by the Young-Fenchel inequality we have that the terms within the other brackets are nonnegative, too, and hence, it follows that all the terms within the brackets must be equal to zero. Combining the last statement with the optimality conditions $\left(i^{\prime}\right)-\left(v^{\prime}\right)$ yields

$$
\text { (i) } \begin{aligned}
& \max _{1 \leq j \leq n}\left\{h_{j}\left(\mathcal{T}_{\Omega_{j}, f_{j}}^{C_{j}}(\bar{x})\right)+a_{j}\right\}=\sum_{i \in \bar{I}} \bar{z}_{i}^{*} \mathcal{T}_{\Omega_{i}, f_{i}}^{C_{i}}(\bar{x})-\sum_{r \in \bar{R}} \bar{\lambda}_{r}\left[h_{r}^{*}\left(\frac{\bar{z}_{r}^{*}}{\bar{\lambda}_{r}}\right)-a_{r}\right] \\
= & \sum_{r \in \bar{R}}\left(\bar{\lambda}_{r} h_{r}\left(\mathcal{T}_{\Omega_{r}, f_{r}}^{C_{r}}(\bar{x})\right)+\bar{\lambda}_{r} a_{r}\right),
\end{aligned}
$$

(ii) $\bar{\lambda}_{r} h_{r}^{*}\left(\frac{\bar{z}_{r}^{*}}{\bar{\lambda}_{r}}\right)+\bar{\lambda}_{r} h_{r}\left(\mathcal{T}_{\Omega_{r}, f_{r}}^{C_{r}}(\bar{x})\right)=\bar{z}_{r}^{*} \mathcal{T}_{\Omega_{r}, f_{r}}^{C_{r}}(\bar{x}) \forall r \in \bar{R}$,

(iii) $\bar{z}_{i}^{*} \mathcal{T}_{\Omega_{i}, f_{i}}^{C_{i}}(\bar{x})+\bar{z}_{i}^{*} f_{i}^{*}\left(\frac{1}{\bar{z}_{i}^{*}} \bar{w}_{i}^{*}\right)+\sigma_{\Omega_{i}}\left(\bar{w}_{i}^{*}\right)=\left\langle\bar{w}_{i}^{*}, \bar{x}\right\rangle \forall i \in \bar{I}$,

(iv) $\sum_{i \in \bar{I}}\left\langle\bar{w}_{i}^{*}, \bar{x}\right\rangle=-\sigma_{S}\left(-\sum_{i \in \bar{I}} \bar{w}_{i}^{*}\right)$,

(v) $\sum_{r \in \bar{R}} \bar{\lambda}_{r} \leq 1, \bar{\lambda}_{k}>0, k \in \bar{R}, \bar{\lambda}_{l}=0, l \notin \bar{R}, \bar{z}_{i}^{*}>0, i \in \bar{I}$, and $\bar{z}_{j}^{*}=0, j \notin \bar{I}$,

(vi) $\gamma_{C_{i}^{0}}\left(\bar{w}_{i}^{*}\right) \leq \bar{z}_{i}^{*}, \bar{w}_{i}^{*} \in X^{*}, i \in \bar{I}$, and $\bar{w}_{j}^{*}=0_{X^{*}}, j \notin \bar{I}$.

From conditions (i) and (v) we obtain that

$$
\begin{aligned}
& \max _{1 \leq j \leq n}\left\{h_{j}\left(\mathcal{T}_{\Omega_{j}, f_{j}}^{C_{j}}(\bar{x})\right)+a_{j}\right\}=\sum_{r \in \bar{R}}\left(\bar{\lambda}_{r} h_{r}\left(\mathcal{T}_{\Omega_{r}, f_{r}}^{C_{r}}(\bar{x})\right)+\bar{\lambda}_{r} a_{r}\right) \\
& \quad \leq \sum_{r \in \bar{R}} \bar{\lambda}_{r} \max _{1 \leq j \leq n}\left\{h_{j}\left(\mathcal{T}_{\Omega_{j}, f_{j}}^{C_{j}}(\bar{x})\right)+a_{j}\right\} \leq \max _{1 \leq j \leq n}\left\{h_{j}\left(\mathcal{T}_{\Omega_{j}, f_{j}}^{C_{j}}(\bar{x})\right)+a_{j}\right\},
\end{aligned}
$$

which means on the one hand that

$$
\sum_{r \in \bar{R}} \bar{\lambda}_{r} \max _{1 \leq j \leq n}\left\{h_{j}\left(\mathcal{T}_{\Omega_{j}, f_{j}}^{C_{j}}(\bar{x})\right)+a_{j}\right\}=\max _{1 \leq j \leq n}\left\{h_{j}\left(\mathcal{T}_{\Omega_{j}, f_{j}}^{C_{j}}(\bar{x})\right)+a_{j}\right\},
$$


i.e. condition (v) can be written as

$$
\sum_{r \in \bar{R}} \bar{\lambda}_{r}=1, \bar{\lambda}_{k}>0, k \in \bar{R}, \bar{\lambda}_{l}=0, l \notin \bar{R}, \bar{z}_{i}^{*}>0, i \in \bar{I} \text {, and } \bar{z}_{j}^{*}=0, j \notin \bar{I},
$$

and on the other hand that

$$
\sum_{r \in \bar{R}}\left(\bar{\lambda}_{r} h_{r}\left(\mathcal{T}_{\Omega_{r}, f_{r}}^{C_{r}}(\bar{x})\right)+\bar{\lambda}_{r} a_{r}\right)=\sum_{r \in \bar{R}} \bar{\lambda}_{r} \max _{1 \leq j \leq n}\left\{h_{j}\left(\mathcal{T}_{\Omega_{j}, f_{j}}^{C_{j}}(\bar{x})\right)+a_{j}\right\}
$$

or, equivalently,

$$
\sum_{r \in \bar{R}} \bar{\lambda}_{r}\left[\max _{1 \leq j \leq n}\left\{h_{j}\left(\mathcal{T}_{\Omega_{j}, f_{j}}^{C_{j}}(\bar{x})\right)+a_{j}\right\}-h_{r}\left(\mathcal{T}_{\Omega_{r}, f_{r}}^{C_{r}}(\bar{x})\right)+a_{r}\right]=0 .
$$

As the brackets in (10) are nonnegative and $\bar{\lambda}_{r}>0$ for $r \in \bar{R}$, it follows that the terms inside the brackets must be equal to zero, more precisely,

$$
\max _{1 \leq j \leq n}\left\{h_{j}\left(\mathcal{T}_{\Omega_{j}, f_{j}}^{C_{j}}(\bar{x})\right)+a_{j}\right\}=h_{r}\left(\mathcal{T}_{\Omega_{r}, f_{r}}^{C_{r}}(\bar{x})\right)+a_{r} \quad \forall r \in \bar{R} .
$$

Further, Theorem 2.1 implies the existence of $\bar{p}_{i}, \bar{q}_{i} \in X$ such that

$$
\mathcal{T}_{\Omega_{i}, f_{i}}^{C_{i}}(\bar{x})=\gamma_{C_{i}}\left(\bar{x}-\bar{p}_{i}-\bar{q}_{i}\right)+f_{i}\left(\bar{p}_{i}\right)+\delta_{\Omega_{i}}\left(\bar{q}_{i}\right) \quad \forall i=1, \ldots, n .
$$

Employing the condition (iii) one gets

$$
\bar{z}_{i}^{*} \gamma_{C_{i}}\left(\bar{x}-\bar{p}_{i}-\bar{q}_{i}\right)-\bar{z}_{i}^{*} f_{i}\left(\bar{p}_{i}\right)-\delta_{\Omega_{i}}\left(\bar{q}_{i}\right)+\bar{z}_{i}^{*} f_{i}^{*}\left(\frac{1}{\bar{z}_{i}^{*}} \bar{w}_{i}^{*}\right)+\sigma_{\Omega_{i}}\left(\bar{w}_{i}^{*}\right)=\left\langle\bar{w}_{i}^{*}, \bar{x}\right\rangle,
$$

equivalently writable as

$$
\begin{aligned}
& {\left[\bar{z}_{i}^{*} \gamma_{C_{i}}\left(\bar{x}-\bar{p}_{i}-\bar{q}_{i}\right)-\left\langle\bar{w}_{i}^{*}, \bar{x}-\bar{p}_{i}-\bar{q}_{i}\right\rangle\right]+\left[\bar{z}_{i}^{*} f_{i}\left(\bar{p}_{i}\right)+\bar{z}_{i}^{*} f_{i}^{*}\left(\frac{1}{\bar{z}_{i}^{*}} \bar{w}_{i}^{*}\right)-\left\langle\bar{w}_{i}^{*}, \bar{p}_{i}\right\rangle\right]} \\
& \quad+\left[\delta_{\Omega_{i}}\left(\bar{q}_{i}\right)+\sigma_{\Omega_{i}}\left(\bar{w}_{i}^{*}\right)-\left\langle\bar{w}_{i}^{*}, \bar{q}_{i}\right\rangle\right], i \in \bar{I} .
\end{aligned}
$$

By the Young-Fenchel inequality all the brackets in (12) are nonnegative and must therefore be equal to zero, i.e.

$$
\begin{aligned}
\bar{z}_{i}^{*} \gamma_{C_{i}}\left(\bar{x}-\bar{p}_{i}-\bar{q}_{i}\right) & =\left\langle\bar{w}_{i}^{*}, \bar{x}-\bar{p}_{i}-\bar{q}_{i}\right\rangle, \\
\bar{z}_{i}^{*} f_{i}\left(\bar{p}_{i}\right)+\bar{z}_{i}^{*} f_{i}^{*}\left(\frac{1}{\bar{z}_{i}^{*}} \bar{w}_{i}^{*}\right) & =\left\langle\bar{w}_{i}^{*}, \bar{p}_{i}\right\rangle, \\
\delta_{\Omega_{i}}\left(\bar{q}_{i}\right)+\sigma_{\Omega_{i}}\left(\bar{w}_{i}^{*}\right) & =\left\langle\bar{w}_{i}^{*}, \bar{q}_{i}\right\rangle, i \in \bar{I} .
\end{aligned}
$$

Now, by (13), condition (vi) and Lemma 2.1 (the generalized Cauchy-Schwarz inequality) yield

$$
\bar{z}_{i}^{*} \gamma_{C_{i}}\left(\bar{x}-\bar{p}_{i}-\bar{q}_{i}\right)=\left\langle\bar{w}_{i}^{*}, \bar{x}-\bar{p}_{i}-\bar{q}_{i}\right\rangle \leq \gamma_{C_{i}^{0}}\left(\bar{w}_{i}^{*}\right) \gamma_{C_{i}}\left(\bar{x}-\bar{p}_{i}-\bar{q}_{i}\right) \leq \bar{z}_{i}^{*} \gamma_{C_{i}}\left(\bar{x}-\bar{p}_{i}-\bar{q}_{i}\right),
$$

which means that condition (vi) can be expressed as

$$
\gamma_{C_{i}^{0}}\left(\bar{w}_{i}^{*}\right)=\bar{z}_{i}^{*}, \bar{w}_{i}^{*} \in X^{*} \backslash\left\{0_{X^{*}}\right\}, i \in \bar{I} \text {, and } \bar{w}_{j}^{*}=0_{X^{*}}, j \notin \bar{I} .
$$

The optimality conditions (i)-(vi), (8), (11) and (14) deliver the desired statement.

(b) All the calculations done in $(a)$ can also be made in the reverse order, yielding thus the conclusion. 
Remark 3.5 If we consider the situation when the set-up costs are arbitrary, i.e. $a_{i}$ can also be negative, $i=1, \ldots, n$, then the conjugate function of $f$ looks like (see [27, Remark 6])

$$
f^{*}\left(z_{1}^{*}, \ldots, z_{n}^{*}\right)=\min _{\substack{i=1 \\ \sum_{i=1}^{n} \lambda_{i}=1, \lambda_{i} \geq 0, i=1, \ldots, n}}\left\{\sum_{i=1}^{n}\left[\left(\lambda_{i} h_{i}\right)^{*}\left(z_{i}^{*}\right)-\lambda_{i} a_{i}\right]\right\} .
$$

As a consequence, the corresponding dual problem turns out to be almost the same one as in (7) with the additional constraint $\sum_{r \in R} \lambda_{r}=1$ and all the statements given in this subsection can be easily adapted for this general case where the set-up costs are arbitrary.

\subsection{Special case I}

We study now the location problem involved in the economical scenario discussed in Remark 3.1 (we set $C_{i}=-C_{i}$ ), i.e.

$$
\left(P_{\gamma_{G}}, \mathcal{T}\right) \inf _{x \in X} \max _{1 \leq i \leq n}\left\{\mathcal{T}_{\Omega_{i}, \gamma_{G_{i}}}^{C_{i}}(x)\right\},
$$

and its dual problem (cf. Proposition 3.1, note that $S=X, a_{i}=0$ and $f_{i}^{*}=\delta_{G_{i}^{0}}, i=$ $1, \ldots, n)$

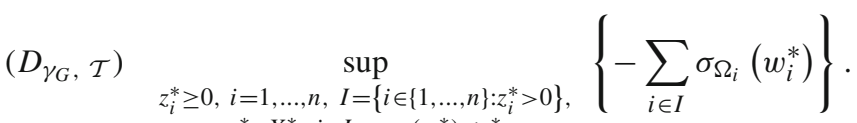

$$
\begin{aligned}
& w_{i}^{*} \in X^{*}, i \in I, \gamma_{C_{i}^{0}}\left(w_{i}^{*}\right) \leq z_{i}^{*}, \\
& \gamma_{G_{i}^{0}}\left(w_{i}^{*}\right) \leq z_{i}^{*}, i \in I, \sum_{i \in I} z_{i}^{*} \leq 1, \quad \sum_{i \in I} w_{i}^{*}=0_{X^{*}}
\end{aligned}
$$

Theorem 3.1 yields the following duality statement for the primal-dual pair $\left(P_{\gamma_{G}}, \mathcal{T}\right)$ $\left(D_{\gamma_{G}}, \mathcal{T}\right)$.

Theorem 3.3 (Strong duality) Between $\left(P_{\gamma_{G}}, \mathcal{T}\right)$ and $\left(D_{\gamma_{G}}, \mathcal{T}\right)$ holds strong duality, i.e. $v\left(P_{\gamma_{G}}, \mathcal{T}\right)=v\left(D_{\gamma_{G}}, \mathcal{T}\right)$ and the dual problem has an optimal solution.

The necessary and sufficient optimality conditions for the primal-dual pair of optimization problems $\left(P_{\gamma_{G}}, \mathcal{T}\right)-\left(D_{\gamma_{G}}, \mathcal{T}\right)$ can be obtained by using the same ideas as in Theorem 3.2.

Theorem 3.4 (Optimality conditions) (a) Let $\bar{x} \in X$ be an optimal solution to the problem $\left(P_{\gamma_{G}}, \mathcal{T}\right)$. Then there exists an optimal solution to $\left(D_{\gamma_{G}}, \mathcal{T}\right)\left(\bar{z}_{1}^{*}, \ldots, \bar{z}_{n}^{*}, \bar{w}_{1}^{*}, \ldots, \bar{w}_{n}^{*}\right)$ with the corresponding index set $\bar{I} \subseteq\{1, \ldots, n\}$ such that

(i) $\max _{1 \leq j \leq n}\left\{\mathcal{T}_{\Omega_{j}, \gamma_{G_{j}}}^{C_{j}}(\bar{x})\right\}=\sum_{i \in \bar{I}} \bar{z}_{i}^{*} \mathcal{T}_{\Omega_{i}, \gamma_{G_{i}}}^{C_{i}}(\bar{x})$,

(ii) $\bar{z}_{i}^{*} \mathcal{T}_{\Omega_{i}, \gamma_{G_{i}}}^{C_{i}}(\bar{x})+\sigma_{\Omega_{i}}\left(\bar{w}_{i}^{*}\right)=\left\langle\bar{w}_{i}^{*}, \bar{x}\right\rangle \quad \forall i \in \bar{I}$,

(iii) $\sum_{i \in \bar{I}} \bar{w}_{i}^{*}=0_{X^{*}}$,

(iv) $\max _{1 \leq j \leq n}\left\{\mathcal{T}_{\Omega_{j}, \gamma_{G_{j}}}^{C_{j}}(\bar{x})\right\}=\mathcal{T}_{\Omega_{i}, \gamma_{G_{i}}}^{C_{i}}(\bar{x}) \quad \forall i \in \bar{I}$,

(v) $\sum_{i \in \bar{I}} \bar{z}_{i}^{*}=1, \bar{z}_{i}^{*}>0, i \in \bar{I}$, and $\bar{z}_{j}^{*}=0, j \notin \bar{I}$,

(vi) $\gamma_{C_{i}^{0}}\left(\bar{w}_{i}^{*}\right)=\bar{z}_{i}^{*}, \bar{w}_{i}^{*} \in X^{*} \backslash\left\{0_{X^{*}}\right\}, \gamma_{G_{i}^{0}}\left(\bar{w}_{i}^{*}\right) \leq \gamma_{C_{i}^{0}}\left(\bar{w}_{i}^{*}\right), i \in \bar{I}$.

(b) If there exists $\bar{x} \in \mathcal{H}$ such that for some $\left(\bar{z}_{1}^{*}, \ldots, \bar{z}_{n}^{*}, \bar{w}_{1}^{*}, \ldots, \bar{w}_{n}^{*}\right)$ and the corresponding index set $\bar{I}$ the conditions $(i)-(v i)$ are fulfilled, then $\bar{x}$ is an optimal solution to $\left(P_{\gamma_{G}}, \mathcal{T}\right)$, $\left(\bar{z}_{1}^{*}, \ldots, \bar{z}_{n}^{*}, \bar{w}_{1}^{*}, \ldots, \bar{w}_{n}^{*}\right)$ is an optimal solution to $\left(D_{\gamma_{G}}, \mathcal{T}\right)$ and $v\left(P_{\gamma_{G}}, \mathcal{T}\right)=v\left(D_{\gamma_{G}}, \mathcal{T}\right)$. 
Proof As $h_{i}^{*}=\delta_{(-\infty, 1]}$ for all $i=1, \ldots, n$, one has from the optimality condition ( $\left.i i\right)$ of Theorem 3.2 that $\bar{z}_{r}^{*} \mathcal{T}_{\Omega_{r}, \gamma_{G_{r}}}^{C_{r}}(\bar{x})=\bar{\lambda}_{r}^{*} \mathcal{T}_{\Omega_{r}, \gamma_{G_{r}}}^{C_{r}}(\bar{x})$ for all $r \in \bar{R}$, which in turn yields that $\bar{I}=\bar{R}$ and $\bar{\lambda}_{i}=\bar{z}_{i}^{*}$ for all $i \in \bar{I}$ (as $0<z_{r}^{*} \leq \lambda_{r}$ and $\bar{I} \subseteq \bar{R}$ ). Furthermore, as $f_{i}^{*}=\delta_{G_{i}^{0}}$, it follows by the optimality conditions (iii) and (vii) of Theorem 3.2 that $\gamma_{G_{i}^{0}}\left(\bar{w}_{i}^{*}\right) \leq \gamma_{C_{i}^{0}}\left(\bar{w}_{i}^{*}\right)$ for all $i \in \bar{I}$. Summing up these facts with the optimality conditions of Theorem 3.2 yields the desired statement.

We use the optimality conditions listed in Theorem 3.4 to provide a more exact characterization to the optimal solutions to the optimization problem $\left(P_{\gamma_{G}}, \mathcal{T}\right)$.

Theorem 3.5 Let $\cap_{i \in \bar{I}} \Omega_{i}=\emptyset, 0 \in \operatorname{int} G_{i}, C_{i}^{0} \cap G_{i} \cap \operatorname{dom} \sigma_{\Omega_{i}} \neq \emptyset$ for all $i \in \bar{I}$, and $\bar{x} \in X$ be an optimal solution to the optimization problem $\left(P_{\gamma_{G}}, \mathcal{T}\right)$. If $\left(\bar{z}_{1}^{*}, \ldots, \bar{z}_{n}^{*}, \bar{w}_{1}^{*}, \ldots, \bar{w}_{n}^{*}\right) \in$ $\mathbb{R}_{+}^{n} \times\left(X^{*}\right)^{n}$ is an optimal solution to $\left(D_{\gamma_{G}}, \mathcal{T}\right)$ with the corresponding $\bar{I} \subseteq\{1, \ldots, n\}$, then

$$
\bar{x} \in \bigcap_{i \in \bar{I}}\left[\partial\left(v\left(D_{\gamma_{G}}, \mathcal{T}\right) \gamma_{C_{i}^{0}}\right)\left(\bar{w}_{i}^{*}\right)+\partial \sigma_{\Omega_{i}}\left(\bar{w}_{i}^{*}\right)\right] .
$$

Proof From $0_{X} \in \operatorname{int} C_{i}$ and $0_{X} \in \operatorname{int} G_{i}$ follows that $0_{X^{*}} \in \operatorname{int} C_{i}^{0}$ and $0_{X^{*}} \in \operatorname{int} G_{i}^{0}$ such that $\gamma_{C_{i}^{0}}$ and $\gamma_{G_{i}^{0}}$ are continuous for all $i \in \bar{I}$. Hence, Theorem 2.1 secures the existence of $\phi_{i} \in X$ and $\psi_{i} \in \Omega_{i}$ such that $\mathcal{T}_{\Omega_{i}, \gamma_{G_{i}}}^{C_{i}}(\bar{x})=\gamma_{C_{i}}\left(\bar{x}-\phi_{i}-\psi_{i}\right)+\gamma_{G_{i}}\left(\phi_{i}\right), i \in \bar{I}$. Further, we have by the optimality conditions $(i i)$ and $(i v)$ of Theorem 3.4

$$
\begin{aligned}
& \left(\gamma_{C_{i}}\left(\bar{x}-\phi_{i}-\psi_{i}\right)+\gamma_{G_{i}}\left(\phi_{i}\right)\right) \gamma_{C_{i}^{0}}\left(\bar{w}_{i}^{*}\right)+\sigma_{\Omega_{i}}\left(\bar{w}_{i}^{*}\right)=\left\langle\bar{w}_{i}^{*}, \bar{x}\right\rangle \\
& \quad \Leftrightarrow\left[\gamma_{C_{i}}\left(\bar{x}-\phi_{i}-\psi_{i}\right) \gamma_{C_{i}^{0}}\left(\bar{w}_{i}^{*}\right)-\left\langle\bar{w}_{i}^{*}, \bar{x}-\phi_{i}-\psi_{i}\right\rangle\right]+\left[\gamma_{G_{i}}\left(\phi_{i}\right) \gamma_{C_{i}^{0}}\left(\bar{w}_{i}^{*}\right)-\left\langle\bar{w}_{i}^{*}, \phi_{i}\right\rangle\right] \\
& \quad+\left[\sigma_{\Omega_{i}}\left(\bar{w}_{i}^{*}\right)-\left\langle\bar{w}_{i}^{*}, \psi_{i}\right\rangle\right]=0, \quad i \in \bar{I},
\end{aligned}
$$

from which follows with the Young-Fenchel inequality that

$$
\begin{aligned}
& \bar{x}-\phi_{i}-\psi_{i} \in \partial\left(\gamma_{C_{i}}\left(\bar{x}-\phi_{i}-\psi_{i}\right) \gamma_{C_{i}^{0}}\right)\left(\bar{w}_{i}^{*}\right), \\
& \phi_{i} \in \partial\left(\gamma_{G_{i}}\left(\phi_{i}\right) \gamma_{C_{i}^{0}}\right)\left(\bar{w}_{i}^{*}\right), \\
& \psi_{i} \in \partial \sigma_{\Omega_{i}}\left(\bar{w}_{i}^{*}\right), \quad i \in \bar{I} .
\end{aligned}
$$

If $\gamma_{C_{i}}\left(\bar{x}-\phi_{i}-\psi_{i}\right)+\gamma_{G_{i}}\left(\phi_{i}\right)=0, i \in \bar{I}$, then we have by (15), (16) and (17) that $\bar{x} \in \partial \sigma_{\Omega_{i}}\left(\bar{w}_{i}^{*}\right)$ for all $i \in \bar{I}$, such that $\bar{x} \in \Omega_{i}$ for all $i \in \bar{I}$, which contradicts our assumption.

If there exists $i \in \bar{I}$ such that $\gamma_{C_{i}}\left(\bar{x}-\phi_{i}-\psi_{i}\right)=0$, then $v\left(D_{\gamma_{G}}, \mathcal{T}\right)=\gamma_{G_{i}}\left(\phi_{i}\right)>0$ and we get by (15), (16) and (17) that

$$
\begin{aligned}
\bar{x}-\psi_{i} \in \partial \delta_{X^{*}}\left(\bar{w}_{i}^{*}\right)+\partial\left(\gamma_{G_{i}}\left(\phi_{i}\right) \gamma_{C_{i}^{0}}\right)\left(\bar{w}_{i}^{*}\right) & =\left\{0_{X^{*}}\right\}+\gamma_{G_{i}}\left(\phi_{i}\right) \partial \gamma_{C_{i}^{0}}\left(\bar{w}_{i}^{*}\right) \\
& =v\left(D_{\gamma_{G}}, \mathcal{T}\right) \partial \gamma_{C_{i}^{0}}\left(\bar{w}_{i}^{*}\right) .
\end{aligned}
$$

If there exists $i \in \bar{I}$ such that $\gamma_{C_{i}}\left(\bar{x}-\phi_{i}-\psi_{i}\right)>0$ and $\gamma_{G_{i}}\left(\phi_{i}\right)=0$, then it follows in a similar way by (15), (16) and (17) that

$$
\bar{x}-\psi_{i} \in v\left(D_{\gamma_{G}}, \mathcal{T}\right) \partial \gamma_{C_{i}^{0}}\left(\bar{w}_{i}^{*}\right) .
$$

Finally, if there exists $i \in \bar{I}$ such that $\gamma_{C_{i}}\left(\bar{x}-\phi_{i}-\psi_{i}\right)>0$ and $\gamma_{G_{i}}\left(\phi_{i}\right)>0$, then one has by (15), (16) and (17) that

$$
\bar{x}-\psi_{i} \in \partial\left(\left(\gamma_{C_{i}}\left(\bar{x}-\phi_{i}-\psi_{i}\right)+\gamma_{G_{i}}\left(\phi_{i}\right)\right) \gamma_{C_{i}^{0}}\right)\left(\bar{w}_{i}^{*}\right)=v\left(D_{\gamma_{G}}, \mathcal{T}\right) \partial \gamma_{C_{i}^{0}}\left(\bar{w}_{i}^{*}\right) .
$$


In summary, we have $\bar{x}-\psi \in v\left(D_{\gamma_{G}}, \mathcal{T}\right) \partial \gamma_{C_{i}^{0}}\left(\bar{w}_{i}^{*}\right)$, which implies that $\bar{x} \in v\left(D_{\gamma_{G}}, \mathcal{T}\right) \partial \gamma_{C_{i}^{0}}\left(\bar{w}_{i}^{*}\right)+\partial \sigma_{\Omega_{i}}\left(\bar{w}_{i}^{*}\right)$ for all $i \in \bar{I}$.

Remark 3.6 Let $\mathcal{H}$ be a real Hilbert space, $\beta_{i}>0, p_{i} \in \mathcal{H}, C_{i}=\left\{x \in \mathcal{H}: \beta_{i}\|x\|_{\mathcal{H}} \leq 1\right\}, \gamma_{G_{i}}$ a norm and $\Omega_{i}=\left\{p_{i}\right\}, i=1, \ldots, n$, with $p_{1}, \ldots, p_{n}$ distinct, then one has by Theorem 3.5

$$
\bar{x}=\frac{v\left(D_{\left.\gamma_{G}, \mathcal{T}\right)}\right.}{\beta_{i}\left\|\bar{w}_{i}^{*}\right\|_{\mathcal{H}}} \bar{w}_{i}^{*}+p_{i} \quad \forall i \in \bar{I} .
$$

Note that if $v\left(P_{\gamma_{G}}, \mathcal{T}\right)=0$, then $\gamma_{C_{i}}\left(\bar{x}-p_{i}-\bar{z}_{i}\right)+\gamma_{G_{i}}\left(\bar{z}_{i}\right)=0$ for all $i=1, \ldots, n$, which means that $z_{i}=0_{\mathcal{H}}$ and $\bar{x}=p_{i}$ for all $i=1, \ldots, n$, i.e. one gets a contradiction. Therefore, taking into consideration that $v\left(P_{\gamma_{G}}, \mathcal{T}\right)>0$ and the strong duality statement, one gets $v\left(D_{\gamma_{G}}, \mathcal{T}\right)>0$ and by the optimality condition (iii) of Theorem 3.4 follows

$$
\sum_{i \in \bar{I}} \frac{\beta_{i}\left\|\bar{w}_{i}^{*}\right\|_{\mathcal{H}}}{v\left(D_{\gamma_{G}}, \mathcal{T}\right)}\left(\bar{x}-p_{i}\right)=\sum_{i \in \bar{I}} \bar{w}_{i}^{*}=0_{\mathcal{H}} \Leftrightarrow \bar{x}=\frac{1}{\sum_{i \in \bar{I}} \beta_{i}\left\|\bar{w}_{i}^{*}\right\|_{\mathcal{H}}} \sum_{i \in \bar{I}} \beta_{i}\left\|\bar{w}_{i}^{*}\right\|_{\mathcal{H}} p_{i} .
$$

Remark 3.7 Let $\cap_{i \in \bar{I}} \Omega_{i}=\emptyset, 0 \in$ int $G_{i}$ and $\gamma_{C_{i}^{0}}(x)=\gamma_{G_{i}^{0}}(x)=0$ if and only if $x=0_{X}$, $i=1, \ldots, n$.

(i) Following Proposition 3.2, the dual problem $\left(\widetilde{D}_{\gamma_{G}}, \mathcal{T}\right)$ can be rewritten as

$$
\begin{gathered}
\left(\widetilde{D}_{\gamma_{G}, \mathcal{T}}\right) \underset{y_{i}^{*} \in X^{*}, i=1, \ldots, n, \sum_{i=1}^{n} y_{i}^{*}=0_{X^{*}},}{\sup _{i=1}^{n}}\left\{-\sum_{\Omega_{i}}\left(y_{i}^{*}\right)\right\}, \\
\sum_{i=1}^{n} \max \left\{\gamma_{\left.C_{i}^{0}\left(y_{i}^{*}\right), \gamma_{G_{i}^{0}}\left(y_{i}^{*}\right)\right\} \leq 1}\right.
\end{gathered}
$$

consequently $v\left(P_{\gamma_{G}}, \mathcal{T}\right)=v\left(\widetilde{D}_{\gamma_{G}}, \mathcal{T}\right)$.

(ii) As the Slater constraint qualification corresponding to $\left(\widetilde{D}_{\gamma_{G}}, \mathcal{T}\right)$ is fulfilled (for instance for $\left.y_{i}^{*}=0_{X^{*}}, i=1, \ldots, n\right)$, there holds strong duality for it and its Lagrange dual problem, that can be reduced after some calculations to

$$
\left(D \widetilde{D}_{\gamma_{G}, \mathcal{T}}\right) \inf _{\lambda \geq 0, x \in X}\left\{\lambda+\sum_{i=1}^{n} \sup _{y_{i}^{*} \in X^{*}}\left\{\left\langle x, y_{i}^{*}\right\rangle-\lambda \max \left\{\gamma_{C_{i}^{0}}\left(y_{i}^{*}\right), \gamma_{G_{i}^{0}}\left(y_{i}^{*}\right)\right\}-\sigma_{\Omega_{i}}\left(y_{i}^{*}\right)\right\}\right\} .
$$

Since $\lambda=0$ implies, taking into consideration that $\cap_{i=1}^{n} \Omega_{i}=\emptyset$, that the value of the objective function of $\left(D \widetilde{D}_{\gamma_{G}}, \mathcal{T}\right)$ is $+\infty$, one can write $\lambda>0$ in the constraints of $\left(D \widetilde{D}_{\gamma_{G}}, \mathcal{T}\right)$. Moreover, since $0_{X^{*}} \in \operatorname{dom} \gamma_{C_{i}^{0}} \cap \operatorname{dom} \gamma_{G_{i}^{0}} \cap \operatorname{dom} \sigma_{\Omega_{i}}$ and $\sigma_{\Omega_{i}}$ is continuous for all $i=$ $1, \ldots, n,[6$, Theorem 3.5.8.(a)] yields

$$
\begin{aligned}
& \sup _{y_{i}^{*} \in X^{*}}\left\{\left\langle x, y_{i}^{*}\right\rangle-\lambda \max \left\{\gamma_{C_{i}^{0}}\left(y_{i}^{*}\right), \gamma_{G_{i}^{0}}\left(y_{i}^{*}\right)\right\}-\sigma_{\Omega_{i}}\left(y_{i}^{*}\right)\right\} \\
& =\min _{y_{i} \in \Omega}\left\{\lambda \max \left\{\gamma_{C_{i}^{0}}(\cdot), \gamma_{G_{i}^{0}}(\cdot)\right\}^{*}\left(\frac{1}{\lambda}\left(x-y_{i}\right)\right)\right\} .
\end{aligned}
$$

(iii) For any $i=1, \ldots, n$, the conjugate of $\max \left\{\gamma_{C_{i}^{0}}(\cdot), \gamma_{G_{i}^{0}}(\cdot)\right\}$ from (19) becomes

$$
\max \left\{\gamma_{C_{i}^{0}}(\cdot), \gamma_{G_{i}^{0}}(\cdot)\right\}^{*}(x)=\sup _{\substack{x^{*} \in X^{*}, t \geq 0, \gamma_{C_{i}^{0}}\left(x^{*}\right) \leq t, \gamma_{G_{i}^{0}}\left(x^{*}\right) \leq t}}\left\{\left\langle x, x^{*}\right\rangle-t\right\}, i=1, \ldots, n .
$$


As the Slater constraint qualification for the problem in the right-hand side of (20) is obviously fulfilled, one obtains via strong Lagrange duality

$$
\max \left\{\gamma_{C_{i}^{0}}(\cdot), \gamma_{G_{i}^{0}}(\cdot)\right\}^{*}(x)=\min _{\substack{\alpha \geq 0, \beta \geq 0, \alpha+\beta \leq 1}}\left(\alpha \gamma_{C_{i}^{0}}+\beta \gamma_{G_{i}^{0}}\right)^{*}(x) .
$$

Note that a more general formula for this conjugate can be found in [7]. Recall that $0_{X} \in \operatorname{int} C_{i}$ and $0_{X} \in \operatorname{int} G_{i}$, which implies that $0_{X^{*}} \in \operatorname{int} C_{i}^{0}$ and $0_{X^{*}} \in \operatorname{int} G_{i}^{0}$ and thus, $\operatorname{dom} \gamma_{C_{i}^{0}}=$ $\operatorname{dom} \gamma_{G_{i}^{0}}=X^{*}$. Hence, we have $0 \cdot \gamma_{C_{i}^{0}}=0 \cdot \gamma_{G_{i}^{0}}=\delta_{X^{*}}$. We apply [6, Theorem 3.5.8.(a)] to the formula in the right-hand side of (21), where the minimum is assumed to be attained at $(\bar{\alpha}, \bar{\beta})$.

If $\bar{\alpha}=0$ and $\bar{\beta}>0$, then we have

$$
\begin{gathered}
\min _{0 \leq \beta \leq 1}\left(\delta_{X^{*}}+\beta \gamma_{G_{i}^{0}}\right)^{*}(x)=\min _{0<\beta \leq 1}\left\{\delta_{\left\{0_{X}\right\}}\left(x-z_{i}\right)+\beta \gamma_{G_{i}^{0}}^{*}\left(\frac{1}{\beta} z_{i}\right)\right\} \\
=\left\{\begin{array}{ll}
0, & \text { if } 0<\bar{\beta} \leq 1, \gamma_{G_{i}}(x) \leq \bar{\beta}, \\
+\infty, & \text { otherwise }
\end{array}= \begin{cases}0, & \text { if } \gamma_{G_{i}}(x) \leq 1 \\
+\infty, & \text { otherwise }\end{cases} \right.
\end{gathered}
$$

If $\bar{\alpha}>0$ and $\bar{\beta}=0$, then one gets similarly that

$$
\min _{0 \leq \alpha \leq 1}\left(\alpha \gamma_{C_{i}^{0}}+\delta_{X^{*}}\right)^{*}(x)= \begin{cases}0, & \text { if } \gamma_{C_{i}}(x) \leq 1 \\ +\infty, & \text { otherwise }\end{cases}
$$

Finally, when $\bar{\alpha}>0$ and $\bar{\beta}>0$, then

$$
\begin{aligned}
& \min _{\substack{\alpha \geq 0, \beta \geq 0, \alpha+\beta \leq 1}}\left(\alpha \gamma_{C_{i}^{0}}+\beta \gamma_{G_{i}^{0}}\right)^{*}(x)=\min _{z_{i} \in X}\left\{\bar{\alpha} \gamma_{C_{i}^{0}}^{*}\left(\frac{1}{\bar{\alpha}}\left(x-z_{i}\right)\right)+\bar{\beta} \gamma_{G_{i}^{0}}^{*}\left(\frac{1}{\bar{\beta}} z_{i}\right)\right\} \\
& = \begin{cases}0, & \text { if } \gamma_{C_{i}}\left(x-z_{i}\right)+\gamma_{G_{i}}\left(z_{i}\right) \leq 1, \\
+\infty, & \text { otherwise. }\end{cases}
\end{aligned}
$$

As $\gamma_{C_{i}^{0}}(x)=\gamma_{C_{i}^{0}}(x)=0 \Leftrightarrow x=0_{X}$, it follows from (22), (23) and (24) that

$\min _{\substack{\alpha \geq 0, \beta \geq 0, \alpha+\beta \leq 1}}\left(\alpha \gamma_{C_{i}^{0}}+\beta \gamma_{G_{i}^{0}}\right)^{*}(x)= \begin{cases}0, & \text { if } \gamma_{C_{i}}\left(x-z_{i}\right)+\gamma_{G_{i}}\left(z_{i}\right) \leq 1, \quad i=1, \ldots, n . \\ +\infty, & \text { otherwise, }\end{cases}$

(iv) Bringing (18), (19) and (25) together allows us consecutively to reformulate the Lagrange dual problem $\left(D \widetilde{D}_{\gamma_{G}}, \mathcal{T}\right)$ as

$$
\begin{aligned}
& \min _{\substack{\lambda>0, x \in X, y_{i} \in \Omega_{i}, z_{i} \in X, \gamma_{C_{i}}\left(x-y_{i}-z_{i}\right)+\gamma_{G_{i}}\left(z_{i}\right) \leq \lambda, i=1, \ldots, n}} \lambda=\min _{\substack{\lambda>0, x \in X, y_{i} \in \Omega_{i}, z_{i} \in X}}\left\{\gamma_{C_{i}}\left(x-y_{i}-z_{i}\right)+\gamma_{G_{i}}\left(z_{i}\right)\right\} \leq \lambda, i=1, \ldots, n \\
& =\min _{\substack{\lambda>0, x \in X, \mathcal{T}_{\Omega_{i}, \gamma_{G_{i}}}^{C_{i}}(x) \leq \lambda, i=1, \ldots, n}} \lambda=\min _{x \in \mathcal{H}} \max _{1 \leq i \leq n}\left\{\mathcal{T}_{\Omega_{i}, \gamma_{G_{i}}}^{C_{i}}(x)\right\} .
\end{aligned}
$$

This shows on the one hand that the optimization problem $\left(P_{\gamma_{G}}, \mathcal{T}\right)$ has an optimal solution and on the other hand that the Lagrange multipliers $\lambda>0$ and $x \in X$ characterize the optimal objective value and the optimal solution to the problem $\left(P_{\gamma_{G}}, \mathcal{T}\right)$, respectively. Furthermore, this fact also implies that the relation between the primal problem $\left(P_{\gamma_{G}}, \mathcal{T}\right)$, its dual problem 
$\left(\widetilde{D}_{\gamma_{G}}, \mathcal{T}\right)$ and its bidual problem $\left(D \widetilde{D}_{\gamma_{G}}, \mathcal{T}\right)$ is completely symmetric under the considered hypotheses.

Remark 3.8 As noted in Remark 3.2, the problem $\left(P_{\gamma_{G}}, \mathcal{T}\right)$ can also be written as

$$
\begin{aligned}
\left(P_{\gamma_{G}, \mathcal{T}}\right) \inf _{\substack{x \in X, t \in \mathbb{R}, \mathcal{T}_{\Omega_{i}, \gamma_{G_{i}}}^{C_{i}}(x) \leq t, i=1, \ldots, n}} t=\inf _{\substack{x \in X, t \in \mathbb{R},(x, t) \in \operatorname{eppi} \mathcal{T}_{\Omega_{i}, \gamma_{G_{i}}}^{C_{i}}, i=1, \ldots, n}} t=\inf _{x \in X, t \in \mathbb{R}}\left\{t+\sum_{i=1}^{n} \delta_{\text {epi } \left.\mathcal{T}_{\Omega_{i}, \gamma_{G_{i}}}^{C_{i}}(x, t)\right\},}\{\right.
\end{aligned}
$$

to which one can assign the corresponding Fenchel dual problem that can be reduced to

$$
\begin{aligned}
& \left(D_{\gamma_{G}}^{F}, \mathcal{T}\right) \sup _{x_{i}^{*} \in X^{*}, t_{i}^{*} \in \mathbb{R}, i=1, \ldots, n,}\left\{-\sum_{i=1}^{n} \sigma_{\text {epi } \mathcal{T}_{\Omega_{i}, \gamma_{G}}^{C_{i}}}\left(x_{i}^{*}, t_{i}^{*}\right)\right\} . \\
& \sum_{i=1}^{n} x_{i}^{*}=0_{X^{*}}, \quad \sum_{i=1}^{n} t_{i}^{*}=-1
\end{aligned}
$$

Now, let us take a careful look at

$$
\sigma_{\text {epi } \mathcal{T}_{\Omega_{i}, \gamma_{G_{i}}}^{C_{i}}\left(x_{i}^{*}, t_{i}^{*}\right)=\sup _{(x, t) \in X \times \mathbb{R}, \mathcal{T}_{\Omega_{i}, \gamma_{G_{i}}}^{C_{i}}}\{(x) \leq t}\left\{\left\langle x_{i}^{*}, x\right\rangle+t_{i}^{*} t\right\},
$$

and as for fixed $\left(x_{i}^{*}, t_{i}^{*}\right) \in X^{*} \times \mathbb{R}$ the Slater constraint qualification is obviously fulfilled in the right-hand side of (26) one has

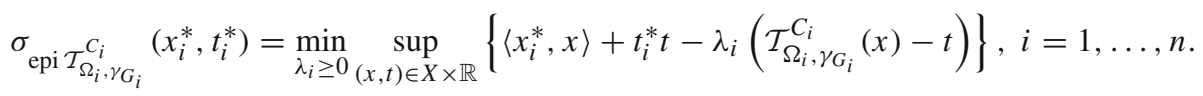

If for some $i \in\{1, \ldots, n\}, \lambda_{i}=0$, then

$$
\sigma_{\text {epi } \mathcal{T}_{\Omega_{i}, \gamma_{G_{i}}}^{C_{i}}}\left(x_{i}^{*}, t_{i}^{*}\right)= \begin{cases}0, & \text { if } x_{i}^{*}=0_{X^{*}}, t_{i}^{*}=0 \\ +\infty, & \text { otherwise, }\end{cases}
$$

otherwise, it holds for some $i \in\{1, \ldots, n\}$,

$$
\begin{gathered}
\sigma_{\text {epi } \mathcal{T}_{\Omega_{i}, \gamma_{G_{i}}}^{C_{i}}\left(x_{i}^{*}, t_{i}^{*}\right)=} \min _{\lambda_{i}>0}\left\{\lambda_{i} \sup _{x \in X}\left\{\left\langle\frac{1}{\lambda_{i}} x_{i}^{*}, x\right\rangle-\mathcal{T}_{\Omega_{i}, \gamma_{G_{i}}}^{C_{i}}(x)\right\}+\sup _{t \in \mathbb{R}}\left\{t\left(t_{i}^{*}+\lambda_{i}\right)\right\}\right\} \\
=\min _{\substack{\lambda_{i}>0, t_{i}^{*}=-\lambda_{i}, \gamma_{C_{i}^{0}}\left(x_{i}^{*}\right) \leq \lambda_{i}, \gamma_{G_{i}^{0}}\left(x_{i}^{*}\right) \leq \lambda_{i}}}\left\{-\sigma_{\Omega_{i}}\left(x_{i}^{*}\right)\right\} .
\end{gathered}
$$

Therefore, the Fenchel dual problem transforms to

$$
\begin{aligned}
& \left(D_{\gamma_{G}}^{F}, \mathcal{T}\right) \sup _{\lambda_{i} \in \mathbb{R}, x_{i}^{*} \in X^{*}, \gamma_{C_{i}^{0}}\left(x_{i}^{*}\right) \leq \lambda_{i}, \gamma_{G_{i}^{0}}\left(x_{i}^{*}\right) \leq \lambda_{i}}\left\{-\sum_{i=1}^{n} \sigma_{\Omega_{i}}\left(x_{i}^{*}\right)\right\} . \\
& i=1, \ldots, n, \sum_{i=1}^{n} \lambda_{i}=1, \sum_{i=1}^{n} x_{i}^{*}=0_{X^{*}}
\end{aligned}
$$


Setting $x_{j}^{*}=w_{j}^{*}, j \in I$, and $\lambda_{i}=z_{i}^{*}, i=1, \ldots, n$, allows to write the Fenchel dual problem as

$$
\begin{aligned}
& \left(D_{\gamma_{G}}^{F}, \mathcal{T}\right) \quad \sup _{z_{i}^{*} \in \mathbb{R}, w_{i}^{*} \in X^{*}, \gamma_{C_{i}^{0}}\left(w_{i}^{*}\right) \leq z_{i}^{*}, \gamma_{G_{i}^{0}}\left(w_{i}^{*}\right) \leq z_{i}^{*}}\left\{-\sum_{i=1}^{n} \sigma_{\Omega_{i}}\left(w_{i}^{*}\right)\right\} . \\
& i=1, \ldots, n, \sum_{i=1}^{n} \lambda_{i}=1, \sum_{i=1}^{n} w_{i}^{*}=0_{X^{*}}
\end{aligned}
$$

More than that, one has by weak duality $v\left(D_{\gamma_{G}}^{F}, \mathcal{T}\right) \leq v\left(D_{\gamma_{G}}, \mathcal{T}\right)=v\left(\widetilde{D}_{\gamma_{G}}, \mathcal{T}\right)=v\left(P_{\gamma_{G}}, \mathcal{T}\right)$.

But this approach has two drawbacks. First, for strong duality between $\left(P_{\gamma_{G}}, \mathcal{T}\right)$ and $\left(D_{\gamma_{G}}^{F}, \mathcal{T}\right)$ one needs to verify the fulfillment of a regularity condition, see, for instance, [6, Theorem 3.2.8]). Moreover, this Fenchel dual cannot be easily reduced to an optimization problem of the form $\left(\widetilde{D}_{\gamma_{G}}, \mathcal{T}\right)$.

Remark 3.9 A further dual problem of interests is the (direct) Lagrange dual to $\left(P_{\gamma_{G}, \mathcal{T}}\right)$

$$
\begin{aligned}
& \left(D_{\gamma_{G}, \mathcal{T}}^{L}\right) \sup _{\lambda_{i} \geq 0, i=1, \ldots, n} \inf _{(x, t) \in X \times \mathbb{R}}\left\{t+\sum_{i=1}^{n} \lambda_{i}\left(\mathcal{T}_{\Omega_{i}, \gamma_{G_{i}}}^{C_{i}}(x)-t\right)\right\} \\
& =\sup _{\lambda_{i} \geq 0, i=1, \ldots, n}\left\{\inf _{x \in X}\left\{\sum_{i=1}^{n} \lambda_{i} \mathcal{T}_{\Omega_{i}, \gamma_{G_{i}}}^{C_{i}}(x)\right\}-\sup _{t \in \mathbb{R}}\left\{t\left(1-\sum_{i=1}^{n} \lambda_{i}\right)\right\}\right\} \\
& =\sup _{\lambda_{i} \geq 0, i=1, \ldots, n, \sum_{i=1}^{n} \lambda_{i}=1} \inf _{x \in X}\left\{\sum_{i=1}^{n} \lambda_{i} \mathcal{T}_{\Omega_{i}, \gamma_{G_{i}}}^{C_{i}}(x)\right\} .
\end{aligned}
$$

The Slater condition is for the problem $\left(\widetilde{P}_{\gamma_{G}}, \mathcal{T}\right)$ fulfilled, i.e. it holds strong duality for the primal-dual pair $\left(\widetilde{P}_{\gamma_{G}}, \mathcal{T}\right)-\left(D_{\gamma_{G}}^{L}, \mathcal{T}\right)$, and from the optimality conditions of Theorem 3.7 follows that $\bar{\lambda} \in \mathbb{R}_{+}^{n}$ with $\bar{\lambda}_{i}=\left(1 / \beta_{i}\right)\left\|_{y_{i}^{*}}^{*}\right\|_{\mathcal{H}}, i \in \bar{I}$, and $\bar{\lambda}_{j}=0, j \notin \bar{I}$, is an optimal solution to the Lagrange dual $\left(D_{\gamma_{G}}^{L}, \mathcal{T}\right)$.

\subsection{Special case II}

In this part of the paper, we analyze the special case where $S=X, a_{i}=0, h_{i}(x):=$ $x+\delta_{\mathbb{R}_{+}}(x), x \in \mathbb{R}, f_{i}(x):=\delta_{L_{i}}(x)$ and $L_{i} \subseteq X$ is a nonempty, closed and convex set for all $i=1, \ldots, n$, such that the minmax location problem $\left(P_{h, \mathcal{T}}^{S}\right)$ turns into

$$
\left(P_{\mathcal{T}}\right) \inf _{x \in X} \max _{1 \leq i \leq n}\left\{\mathcal{T}_{\Omega_{i}, \delta_{L_{i}}}^{C_{i}}(x)\right\},
$$

Remark 3.10 By construction $v\left(P_{\mathcal{T}}\right)>0$. If $X=\mathbb{R}^{m}$ and the gauges are taken to be the corresponding Euclidean norm, then the problem $\left(P_{\mathcal{T}}\right)$ can be seen as finding a point $\bar{x} \in \mathcal{H}$ such that the maximal distance to its Euclidean projections onto the target sets $\Omega_{i}+L_{i}$, $i=1, \ldots, n$, is minimal. If $n=3, X=\mathbb{R}^{2}, \Omega_{i}=\left\{0_{\mathbb{R}^{2}}\right\}$ and $L_{i}=\left\{x \in \mathbb{R}^{2}:\left\|x-p_{i}\right\| \leq a_{i}\right\}$, where $a_{i}>0$ and $p_{i} \in \mathbb{R}^{2}, i=1,2,3$, then this problem is also known as the classical Apollonius problem (see $[4,13,18]$ ). 
The corresponding dual problem $\left(D_{\mathcal{T}}\right)$ to $\left(P_{\mathcal{T}}\right)$ becomes, via Remark 3.4,

$$
\left(D_{\mathcal{T}}\right) \sup _{\substack{I=\left\{i \in\{1, \ldots, n\}: z_{i}^{*}>0\right\}, w_{i}^{*} \in X^{*}, i \in I, z_{i}^{*} \geq 0, \sum_{i}, \ldots, n,}}\left\{-\sum_{i \in I}\left[\sigma_{L_{i}}\left(w_{i}^{*}\right)+\sigma_{\Omega_{i}}\left(w_{i}^{*}\right)\right]\right\} .
$$

Additionally to this dual problem, we consider the following one, that is equivalent to it in the sense that they share the same optimal objective value and this can be proven similarly to the proof of [27, Theorem 9],

$$
\left(\widetilde{D}_{\mathcal{T}} \sup _{\substack{y_{i}^{*} \in X^{*}, \sum_{i \in I} \gamma_{C_{i}^{0}}\left(y_{i}^{*}\right) \leq 1, \ldots, n, I=\left\{i \in\{1, \ldots, n\}: \gamma_{C_{i}^{0}}\left(y_{i}^{*}\right)>0 \\ y_{i}^{*}=0_{X^{*}}\right.}}\left\{-\sum_{i \in I}\left[\sigma_{L_{i}}\left(y_{i}^{*}\right)+\sigma_{\Omega_{i}}\left(y_{i}^{*}\right)\right]\right\} .\right.
$$

Remark 3.11 Considering $\left(\widetilde{D}_{\mathcal{T}}\right)$ in a finitely dimensional setting as a minimization problem, the following economical interpretation arises, where the objective function can be seen as a cost function. The components of the dual variables $y_{i}^{*} \in \mathbb{R}^{m}, i=1, \ldots, n$, express the expected expenditures on public goods and services, where $i$ can be identified as one of $n$ locations. More precisely, every location $i$ has its own vector of $m$ expenditures. Examples of public goods and services can be, for instance, parks, police stations, fire departments or highways. If a component of any vector is zero, then this means that the market (or the citizens) of this location is (are) saturated regarding this good or service and if a component is negative, then the market is supersaturated. The constraint $\sum_{i=1}^{n} \gamma_{C_{i}^{0}}\left(y_{i}^{*}\right) \leq 1$ defines then the limitation of the budget for the public goods and services, while the constraint $\sum_{i=1}^{n} y_{i}^{*}=0_{\mathbb{R}^{m}}$ describes the substitution character of the goods and services. The latter means that if the market $i$ has expected expenditures on a special good or service, then it is taken from an another location which is supersaturated. Therefore, the dual problem $\left(\widetilde{D}_{\mathcal{T}}\right)$ can be understood as a cost minimization problem of the government of $n$ locations, which has to find the optimal allocation of public goods and services $\left(\bar{y}_{1}^{*}, \ldots, \bar{y}_{n}^{*}\right)$ for $n$ locations (that can be districts, towns or federal states) such that all expected expenditures can be financed, the demands on public goods and services of the citizens are saturated and the costs minimal. Another scenario appears by considering the dual problem $\left(\widetilde{D}_{\mathcal{T}}\right)$ as a cost minimization problem of the World Health Organization (WHO), where the components of a vector $z_{i}^{*} \in \mathbb{R}^{m}$ represents the expected expenditures on medical treatment and health care for $m$ diseases for a region $i$ in the world, $i=1, \ldots, n$,. Here also the constraint $\sum_{i=1}^{n} \gamma_{C_{i}^{0}}\left(y_{i}^{*}\right) \leq 1$ characterizes the budget restrictions of the WHO. Moreover, if in a region $i$ for a disease $j \in\{1, \ldots, m\}$ no medical treatment is required and medical staff and products are no longer needed (which means that the associated component $y_{i j}^{*}$ is negative), for instance because a certain disease was eradicated there, then these expenditures can be reallocated to other regions which need medical treatment for the disease $j$. It is important not to waste any expenditures, therefore their sum must be zero, i.e. the constraint $\sum_{i=1}^{n} y_{i}^{*}=0_{\mathbb{R}^{m}}$ must be fulfilled. For an economical interpretation of $\left(P_{\mathcal{T}}\right)$ we refer to [4].

The following statement is then a direct consequence of Theorem 3.1.

Theorem 3.6 (Strong duality) Between $\left(P_{\mathcal{T}}\right)$ and $\left(\widetilde{D}_{\mathcal{T}}\right)$ holds strong duality, i.e. $v\left(P_{\mathcal{T}}\right)=$ $v\left(\widetilde{D}_{\mathcal{T}}\right)$ and the dual problem has an optimal solution $\bar{y}^{*} \in X^{n}$. 
The necessary and sufficient optimality conditions for the primal-dual pair of optimization problems $\left(P_{\mathcal{T}}\right)-\left(\widetilde{D}_{\mathcal{T}}\right)$ can be derived by Theorem 3.6 similarly to the ones in Theorem 3.2.

Theorem 3.7 (Optimality conditions) (a) Let $\bar{x} \in X$ be an optimal solution to the problem $\left(P_{\mathcal{T}}\right)$. Then there exists an optimal solution to $\left(\widetilde{D}_{\mathcal{T}}\right) \bar{y}^{*} \in\left(X^{*}\right)^{n}$ with the corresponding index set $\bar{I} \subseteq\{1, \ldots, n\}$ such that

(i) $\max _{1 \leq j \leq n}\left\{\mathcal{T}_{\Omega_{j}, \delta_{L_{j}}}^{C_{j}}(\bar{x})\right\}=\sum_{i \in \bar{I}} \gamma_{C_{i}^{0}}\left(\bar{y}_{i}^{*}\right) \mathcal{T}_{\Omega_{i}, \delta_{L_{i}}}^{C_{i}}(\bar{x})$,

(ii) $\gamma_{C_{i}^{0}}\left(\bar{y}_{i}^{*}\right) \mathcal{T}_{\Omega_{i}, \delta_{L_{i}}}^{C_{i}}(\bar{x})+\sigma_{L_{i}}\left(\bar{y}_{i}^{*}\right)+\sigma_{\Omega_{i}}\left(\bar{y}_{i}^{*}\right)=\left\langle\bar{y}_{i}^{*}, \bar{x}\right\rangle, i \in \bar{I}$,

(iii) $\sum_{i \in \bar{I}} \bar{y}_{i}^{*}=0_{X^{*}}$

(iv) $\sum_{j \in \bar{I}} \gamma_{C_{i}^{0}}\left(\bar{y}_{i}^{*}\right)=1, \bar{y}_{i}^{*} \in X^{*} \backslash\left\{0_{X^{*}}\right\}, i \in \bar{I}$, and $\bar{y}_{i}^{*}=0_{X^{*}}, i \notin \bar{I}$,

(v) $\mathcal{T}_{\Omega_{i}, \delta_{L_{i}}}^{C_{i}}(\bar{x})=\max _{1 \leq j \leq n}\left\{\mathcal{T}_{\Omega_{j}, \delta_{L_{j}}}^{C_{j}}(\bar{x})\right\}, i \in \bar{I}$.

(b) If there exists $\bar{x} \in X$ such that for some $\left(\bar{y}_{1}^{*}, \ldots, \bar{y}_{n}^{*}\right) \in\left(X^{*}\right)^{n}$ with the corresponding index set $\bar{I}$ the conditions $(i)-(v)$ are fulfilled, then $\bar{x}$ is an optimal solution to $\left(P_{\mathcal{T}}\right)$, $\left(\bar{y}_{1}^{*}, \ldots, \bar{y}_{n}^{*}\right)$ is an optimal solution to $\left(\widetilde{D}_{\mathcal{T}}\right)$ and $v\left(P_{\mathcal{T}}\right)=v\left(\widetilde{D}_{\mathcal{T}}\right)$.

The next statement makes use of Theorem 3.7 in order to provide an exact characterization of the optimal solutions of the optimization problem $\left(P_{\mathcal{T}}\right)$.

Theorem 3.8 Let $\bar{x} \in X$ be an optimal solution to the optimization problem $\left(P_{\mathcal{T}}\right)$. If $\left(\bar{y}_{1}^{*}, \ldots, \bar{y}_{n}^{*}\right) \in\left(X^{*}\right)^{n}$ is an optimal solution to $\left(\widetilde{D}_{\mathcal{T}}\right)$ with the corresponding $\bar{I} \subseteq\{1, \ldots, n\}$, then

$$
\bar{x} \in \bigcap_{i \in \bar{I}}\left[\partial\left(v\left(\widetilde{D}_{\mathcal{T}}\right) \gamma_{C_{i}^{0}}\right)\left(\bar{y}_{i}^{*}\right)+\partial \sigma_{L_{i}}\left(\bar{y}_{i}^{*}\right)+\partial \sigma_{\Omega_{i}}\left(\bar{y}_{i}^{*}\right)\right] .
$$

The proof of Theorem 3.8 is analogous to the one of Theorem 3.5, so we skip it.

In the rest of the paper we assume that $X=\mathcal{H}$, where $\mathcal{H}$ is a real Hilbert space, $\beta_{i}>0$ and $\gamma_{C_{i}}(\cdot)=\beta_{i}\|\cdot\|_{\mathcal{H}}$. Note that in this situation $C_{i}=\left\{x \in \mathcal{H}: \beta_{i}\|x\|_{\mathcal{H}} \leq 1\right\}, X^{*}=\mathcal{H}$ and $\gamma_{C_{i}^{0}}(\cdot)=\left(1 / \beta_{i}\right)\|\cdot\|_{\mathcal{H}}, i=1, \ldots, n$.

Corollary 3.1 Let $\bar{x} \in \mathcal{H}^{n}$ be an optimal solution to the optimization problem $\left(P_{\mathcal{T}}\right)$. If $\left(\bar{y}_{1}^{*}, \ldots, \bar{y}_{n}^{*}\right) \in \mathcal{H}^{n}$ is an optimal solution to $\left(\widetilde{D}_{\mathcal{T}}\right)$ with the corresponding $\bar{I} \subseteq\{1, \ldots, n\}$, then there exist $\phi_{i} \in \Omega_{i}$ and $\psi_{i} \in L_{i}$ fulfilling $\sigma_{L_{i}}\left(\bar{y}_{i}^{*}\right)=\left\langle\bar{y}_{i}^{*}, \psi_{i}\right\rangle$ and $\sigma_{\Omega_{i}}\left(\bar{y}_{i}^{*}\right)=\left\langle\bar{y}_{i}^{*}, \phi_{i}\right\rangle$, i.e. $\psi_{i} \in \partial \sigma_{L_{i}}\left(\bar{y}_{i}^{*}\right)$ and $\phi_{i} \in \partial \sigma_{\Omega_{i}}\left(\bar{y}_{i}^{*}\right), i \in \bar{I}$, such that

$$
\bar{x}=\frac{1}{\sum_{i \in \bar{I}} \beta_{i}\left\|\bar{y}_{i}^{*}\right\|_{\mathcal{H}}} \sum_{i \in \bar{I}} \beta_{i}\left\|\bar{y}_{i}^{*}\right\|_{\mathcal{H}}\left(\phi_{i}+\psi_{i}\right) .
$$

Proof By Theorem 3.8 it holds for each $i \in \bar{I}$

$$
\bar{x} \in \partial\left(v\left(\widetilde{D}_{\mathcal{T}}\right) \frac{1}{\beta_{i}}\|\cdot\| \mathcal{H}\right)\left(\bar{y}_{i}^{*}\right)+\partial \sigma_{L_{i}}\left(\bar{y}_{i}^{*}\right)+\partial \sigma_{\Omega_{i}}\left(\bar{y}_{i}^{*}\right),
$$

which means that there exist $\phi_{i} \in \partial \sigma_{L_{i}}\left(\bar{y}_{i}^{*}\right)$ and $\psi_{i} \in \partial \sigma_{\Omega_{i}}\left(\bar{y}_{i}^{*}\right)$ such that

$$
\bar{x}-\phi_{i}-\psi_{i} \in v\left(\widetilde{D}_{\mathcal{T}}\right) \frac{1}{\beta_{i}} \partial\left(\|\cdot\|_{\mathcal{H}}\right)\left(\bar{y}_{i}^{*}\right) .
$$


As $\bar{y}_{i}^{*} \neq 0_{\mathcal{H}}$, it follows, as $v\left(\widetilde{D}_{\mathcal{T}}\right)>0$ due to Remark 3.6 and Theorem 3.7, that

$$
\frac{\beta_{i}}{v\left(\widetilde{D}_{\mathcal{T}}\right)}\left(\bar{x}-\phi_{i}-\psi_{i}\right)=\frac{1}{\left\|\bar{y}_{i}^{*}\right\|_{\mathcal{H}}} \bar{y}_{i}^{*}, i \in \bar{I} .
$$

Now, we take the sum over all $i \in \bar{I}$ in (27) and get that

$$
\frac{1}{v\left(\widetilde{D}_{\mathcal{T}}\right)} \sum_{i \in \bar{I}} \beta_{i}\left\|\bar{y}_{i}^{*}\right\|_{\mathcal{H}} \bar{x}=\frac{1}{v\left(\widetilde{D}_{\mathcal{T}}\right)} \sum_{i \in \bar{I}} \beta_{i}\left\|\bar{y}_{i}^{*}\right\|_{\mathcal{H}}\left(\phi_{i}+\psi_{i}\right)+\sum_{i \in \bar{I}} \bar{y}_{i}^{*} .
$$

From the optimality condition ( $\mathrm{iii}$ ) of Theorem 3.7 follows that the last term of (28) is equal to zero, which finally yields that

$$
\bar{x}=\frac{1}{\sum_{i \in \bar{I}} \beta_{i}\left\|\bar{y}_{i}^{*}\right\|_{\mathcal{H}}} \sum_{i \in \bar{I}} \beta_{i}\left\|\bar{y}_{i}^{*}\right\|_{\mathcal{H}}\left(\phi_{i}+\psi_{i}\right) .
$$

Remark 3.12 If $\Omega_{i}=\mathcal{H}$ and $L_{i}=\left\{p_{i}\right\}, i=1, \ldots, n$, where $p_{1}, \ldots, p_{n}$ are distinct points in $\mathcal{H}$, then (see [27, Corollary 1])

$$
\bar{x}=\frac{1}{\sum_{i \in \bar{I}} \beta_{i}\left\|\bar{y}_{i}^{*}\right\|_{\mathcal{H}}} \sum_{i \in \bar{I}} \beta_{i}\left\|\bar{y}_{i}^{*}\right\|_{\mathcal{H}} p_{i} .
$$

Remark 3.13 Take note that one can prove in the same way as in the proof of [27, Corollary 2] that for a feasible solution $y^{*}$ to $\left(\widetilde{D}_{\mathcal{T}}\right)$ it holds

$$
\left\|y_{i}^{*}\right\|_{\mathcal{H}} \leq \frac{\beta_{s} \beta_{i}}{\beta_{s}+\beta_{i}}, i \in I,
$$

where $\beta_{s}:=\max _{1 \leq i \leq n}\left\{\beta_{i}\right\}$.

Remark 3.14 Under the assumption that $\beta_{1}=\ldots=\beta_{n}=1$ and $\cap_{i=1}^{n}\left(\Omega_{i}+L_{i}\right)=\emptyset$, any two nonzero components of an optimal solution $\bar{y}^{*}$ to $\left(\widetilde{D}_{\mathcal{T}}\right)$ are linearly independent. To see this, let us assume that there exist $i, j \in \bar{I}, i \neq j$, and $k_{j}>0$ such that $\bar{y}_{i}^{*}=k_{j} \bar{y}_{j}^{*}$. Further, it holds by (27) that

$$
\bar{y}_{i}^{*}=\frac{\left\|\bar{y}_{i}^{*}\right\|_{\mathcal{H}}}{v\left(\widetilde{D}_{\mathcal{T}}\right)}\left(\bar{x}-\phi_{i}-\psi_{i}\right)=k_{j} \bar{y}_{j}^{*}=k_{j} \frac{\left\|\bar{y}_{j}^{*}\right\|_{\mathcal{H}}}{v\left(\widetilde{D}_{\mathcal{T}}\right)}\left(\bar{x}-\phi_{j}-\psi_{j}\right),
$$

from which follows that

$$
\bar{x}-\phi_{i}-\psi_{i}=k_{j} \frac{\left\|\bar{y}_{j}^{*}\right\|_{\mathcal{H}}}{\left\|\bar{y}_{i}^{*}\right\|_{\mathcal{H}}}\left(\bar{x}-\phi_{j}-\psi_{j}\right),
$$

thus one gets that $\bar{x}-\phi_{i}-\psi_{i}=\bar{x}-\phi_{j}-\psi_{j} \Leftrightarrow \phi_{i}+\psi_{i}=\phi_{j}+\psi_{j}$, which contradicts the assumption that $\cap_{i=1}^{n}\left(\Omega_{i}+L_{i}\right)=\emptyset$. Therefore, $\bar{y}_{i}^{*} \neq k_{j} \bar{y}_{j}^{*}, k_{j}>0, i \neq j$, for all $i, j \in \bar{I}$.

Remark 3.15 (i) Clearly, if $\partial \sigma_{\Omega_{i}+L_{i}}\left(\bar{y}_{i}^{*}\right)$ is a singleton for some $i \in \bar{I}$ (which is the situation, when for instance the set $\Omega_{i}+L_{i}$ is strictly convex or its indicator function is Gâteauxdifferentiable at $\left.\bar{y}_{i}^{*}\right)$, then the optimal solution $\bar{x}$ of $\left(P_{\mathcal{T}}\right)$ can be determined immediately by the formula (27), i.e.

$$
\bar{x}=\frac{v\left(\widetilde{D}_{\mathcal{T}}\right)}{\beta_{i}\left\|\bar{y}_{i}^{*}\right\|_{\mathcal{H}}} \bar{y}_{i}^{*}+\phi_{i}+\psi_{i}
$$


(ii) Recall moreover that $v\left(\widetilde{D}_{\mathcal{T}}\right)=v\left(P_{\mathcal{T}}\right)$ due to Theorem 3.6. For instance, take for an index $i \in \bar{I}$ the sets $\Omega_{i}:=\left\{0_{\mathcal{H}}\right\}$ and $L_{i}:=\left\{x \in \mathcal{H}:\left\|x-p_{i}\right\|_{\mathcal{H}} \leq a_{i}\right\}$, where $p_{i} \in \mathcal{H}$ and $a_{i}>0$, then

$$
\begin{aligned}
& \psi_{i} \in \partial \sigma_{L_{i}}\left(\bar{y}_{i}^{*}\right) \Leftrightarrow \psi_{i} \in \partial\left(\frac{1}{a_{i}}\|\cdot\|_{\mathcal{H}}\right)\left(\bar{y}_{i}^{*}\right)+p_{i} \Leftrightarrow a_{i}\left(\psi_{i}-p_{i}\right) \in \partial\left(\|\cdot\|_{\mathcal{H}}\right)\left(\bar{y}_{i}^{*}\right) \\
& \Leftrightarrow a_{i}\left(\psi_{i}-p_{i}\right)=\frac{1}{\left\|\bar{y}_{i}^{*}\right\|_{\mathcal{H}}} \bar{y}_{i}^{*} \Leftrightarrow \psi_{i}=\frac{1}{a_{i}\left\|\bar{y}_{i}^{*}\right\|_{\mathcal{H}}} \bar{y}_{i}^{*}+p_{i},
\end{aligned}
$$

hence

$$
\bar{x}=\left(\frac{v\left(\widetilde{D}_{\mathcal{T}}\right)}{\beta_{i}}+\frac{1}{a_{i}}\right) \frac{1}{\left\|\bar{y}_{i}^{*}\right\|_{\mathcal{H}}} \bar{y}_{i}^{*}+p_{i} .
$$

(iii) Let us consider another situation where $\mathcal{H}=\mathbb{R}^{m},\|\cdot\|_{\infty}$ is the $\infty$-norm, $\|\cdot\|_{1}$ is the $l_{1}$-norm, $\Omega_{i}:=\left\{0_{\mathbb{R}^{m}}\right\}$ and $L_{i}:=\left\{x \in \mathbb{R}^{m}:\left\|x-p_{i}\right\|_{\infty} \leq a_{i}\right\}$. Further, let $\bar{x}=$ $\left(\bar{x}_{1}, \ldots, \bar{x}_{m}\right)^{T} \in \mathbb{R}^{m}, \bar{y}_{i}^{*}=\left(\bar{y}_{i 1}^{*}, \ldots, \bar{y}_{i m}^{*}\right)^{T} \in \mathbb{R}^{m}, \psi_{i}=\left(\psi_{i 1}, \ldots, \psi_{i m}\right)^{T} \in \mathbb{R}^{m}$ and $p_{i}=\left(p_{i 1}, \ldots, p_{i m}\right)^{T} \in \mathbb{R}^{m}$. One has

$$
\begin{aligned}
& \psi_{i} \in \partial \sigma_{L_{i}}\left(\bar{y}_{i}^{*}\right)=\partial\left(a_{i}\|\cdot\|_{1}\right)\left(\bar{y}_{i}^{*}\right)+p_{i} \\
& \Leftrightarrow \frac{1}{a_{i}}\left(\psi_{i}-p_{i}\right) \in \partial\left(\|\cdot\|_{1}\right)\left(\bar{y}_{i}^{*}\right) \Leftrightarrow \frac{1}{a_{i}}\left(\psi_{i j}-p_{i j}\right) \in \begin{cases}\{1\}, & \text { if } \bar{y}_{i j}^{*}>0, \\
\{-1\}, & \text { if } \bar{y}_{i j}^{*}<0, \\
{[-1,1],} & \text { if } \bar{y}_{i j}^{*}=0,\end{cases}
\end{aligned}
$$

$i \in \bar{I}, j=1, \ldots, m$. Now, we define the following index set $J=\left\{j \in\{1, \ldots, m\}: \bar{y}_{i j}^{*}=\right.$ 0 for all $i \in \bar{I}\}$, then it follows for $j \in J$ from (30) that

$$
\frac{1}{a_{i}}\left(\psi_{i j}-p_{i j}\right) \in[-1,1] \Leftrightarrow \psi_{i j} \in p_{i j}+\left[-a_{i}, a_{i}\right] .
$$

Combining (27) and (31) implies that under the corresponding hypotheses it holds

$$
\bar{x}_{j} \in \bigcap_{i \in \bar{I}}\left(p_{i j}+\left[-a_{i}, a_{i}\right]\right), j \in J
$$

Otherwise, if $j \notin J$, there exists $i \in \bar{I}$ such that $\bar{y}_{i j}^{*}>0$ and by (30) it holds

$$
\psi_{i j}= \begin{cases}a_{i}+p_{i j}, & \text { if } \bar{y}_{i j}^{*}>0 \\ -a_{i}+p_{i j}, & \text { if } \bar{y}_{i j}^{*}<0\end{cases}
$$

and hence, one gets by (27) and (32) that

$$
\bar{x}_{j}= \begin{cases}\frac{v\left(\widetilde{D}_{\mathcal{T}}\right)}{\beta_{i}\left\|\bar{y}_{i}^{*}\right\|} \bar{y}_{i j}^{*}+a_{i}+p_{i j}, & \text { if } \bar{y}_{i j}^{*}>0, \\ \frac{v\left(\widetilde{D}_{\mathcal{T}}\right)}{\beta_{i}\left\|\bar{y}_{i}^{*}\right\|} \bar{y}_{i j}^{*}-a_{i}+p_{i j}, & \text { if } \bar{y}_{i j}^{*}<0 .\end{cases}
$$

(iv) Finally, when $m=2,|\bar{I}|>2$ and $\beta_{1}=\ldots=\beta_{n}=1$, it follows by Remark 3.14 that $J=\emptyset$ and hence, there exist $i, j \in \bar{I}$ with $\bar{y}_{i 1}^{*} \neq 0$ and $\bar{y}_{j 2}^{*} \neq 0$ such that

$$
\bar{x}_{1}=\left\{\begin{array}{ll}
\frac{v\left(\widetilde{D}_{\mathcal{T}}\right)}{\left\|\bar{y}_{j}^{*}\right\|} \bar{y}_{i 1}^{*}+a_{i}+p_{i 1}, & \text { if } \bar{y}_{i 1}^{*}>0, \\
\frac{v\left(\widetilde{D}_{\mathcal{T}}\right)}{\left\|\bar{y}_{i}^{*}\right\|} \bar{y}_{i 1}^{*}-a_{i}+p_{i 1}, & \text { if } \bar{y}_{i 1}^{*}<0,
\end{array} \text { and } \bar{x}_{2}= \begin{cases}\frac{v\left(\widetilde{D}_{\mathcal{T}}\right)}{\left\|\bar{y}_{j}^{*}\right\|} \bar{y}_{j 2}^{*}+a_{j}+p_{j 2}, & \text { if } \bar{y}_{j 2}^{*}>0, \\
\frac{v\left(\widetilde{D}_{\mathcal{T}}\right)}{\left\|\bar{y}_{j}^{*}\right\|} \bar{y}_{j 2}^{*}-a_{j}+p_{j 2}, & \text { if } \bar{y}_{j 2}^{*}<0 .\end{cases}\right.
$$


Remark 3.16 The optimality conditions of Theorem 3.7 allow to give the following geometrical interpretation of the set of optimal solutions to the conjugate dual problem $\left(\widetilde{D}_{\mathcal{T}}\right)$ in the situation when $\mathcal{H}=\mathbb{R}^{m}$. When $\gamma_{C_{i}}(\cdot)=\|\cdot\|$ one gets from condition (ii) of Theorem 3.7 via the Young-Fenchel inequality $\left\|\bar{y}_{i}^{*}\right\|\left\|\bar{x}-\phi_{i}-\psi_{i}\right\|=\left\langle\bar{y}_{i}^{*}, \bar{x}-\phi_{i}-\psi_{i}\right\rangle$, which means that the vectors $\bar{y}_{i}^{*}$ and $\bar{x}-\phi_{i}-\psi_{i}, i \in \bar{I}$, are parallel and directed to $\bar{x}$. Further, from the optimal condition $(v)$ of Theorem 3.7 one gets that $i \in \bar{I}$, i.e. $\bar{y}_{i}^{*} \neq 0_{\mathcal{H}}$, if the points $\phi_{i}+\psi_{i} \in \Omega_{i}+L_{i}$ are lying on the boundary of the ball centered at $\bar{x}$ with radius $\left(\widetilde{D}_{\mathcal{T}}\right)$. If $i \notin \bar{I}$, i.e. $\bar{y}_{i}^{*}=0_{\mathcal{H}}$, then the points $\phi_{i}+\psi_{i} \in \Omega_{i}+L_{i}$ are lying on the border of a ball centered at $\bar{x}$ with radius $t<v\left(\widetilde{D}_{\mathcal{T}}\right)$. Hence, the vectors $\bar{y}_{i}^{*}, i \in \bar{I}$, can be interpreted as force vectors, which pull the sets $\Omega_{i}+L_{i}, i \in \bar{I}$, in direction of the center, the gravity point $\bar{x}$, to reduce the minimal time needed to reach the farthest set(s) (see Fig. 2).

We close this section with a statement which gives a formula of the projection operator onto the epigraph of the maximum of norms needed for our numerical experiments.

Theorem 3.9 Let $\gamma_{C}: \mathcal{H}_{1} \times \ldots \times \mathcal{H}_{n} \rightarrow \mathbb{R}$ be defined by $\gamma_{C}\left(x_{1}, \ldots, x_{n}\right):=$ $\max _{1 \leq i \leq n}\left\{\left(\left\|x_{i}\right\|_{\mathcal{H}_{i}}\right) / w_{i}\right\}$, with $w_{i}>0, i=1, \ldots, n$, then it holds

$$
\mathrm{P}_{\mathrm{epi} \gamma_{C}}\left(x_{1}, \ldots, x_{n}, \xi\right)= \begin{cases}\left(x_{1}, \ldots, x_{n}\right), & \text { if } \max _{1 \leq i \leq n}\left\{\frac{1}{w_{i}}\left\|x_{i}\right\|_{\left.\mathcal{H}_{i}\right\} \leq \xi},\right. \\ \left(0_{\mathcal{H}_{1}}, \ldots, 0_{\mathcal{H}_{n}}, 0\right), & \text { if } \xi<0 \text { and } \sum_{i=1}^{n} w_{i}\left\|_{x_{i}}\right\|_{\mathcal{H}_{i}} \leq-\xi \\ \left(\bar{y}_{1}, \ldots, \bar{y}_{n}, \bar{\theta}\right), & \text { otherwise, }\end{cases}
$$

where

$$
\bar{y}_{i}=x_{i}-\frac{\max \left\{\left\|x_{i}\right\|_{\mathcal{H}_{i}}-(\bar{\kappa}+\xi) w_{i}, 0\right\}}{\left\|x_{i}\right\|_{\mathcal{H}_{i}}} x_{i}, i=1, \ldots, n, \text { and } \bar{\theta}=\frac{\sum_{i=k+1}^{n} w_{i}^{2} \tau_{i}+\xi}{\sum_{i=k+1}^{n} w_{i}^{2}+1}
$$

with

$$
\bar{\kappa}=\frac{\sum_{i=k+1}^{n} w_{i}^{2} \tau_{i}-\xi \sum_{i=k+1}^{n} w_{i}^{2}}{\sum_{i=k+1}^{n} w_{i}^{2}+1}
$$

and $k \in\{0,1, \ldots, n-1\}$ is the unique integer such that $\tau_{k}+\xi \leq \bar{\kappa} \leq \tau_{k+1}+\xi$, where the values $\tau_{0}, \ldots, \tau_{n}$ are defined by $\tau_{0}:=0$ and $\tau_{i}:=\left(\left\|x_{i}\right\|_{\mathcal{H}_{i}}\right) / w_{i}, i=1, \ldots, n$, and in ascending order.

Proof As $C=\left\{\left(x_{1}, \ldots, x_{n}\right): \mathcal{H}_{1} \times \ldots \times \mathcal{H}_{n}: \max _{1 \leq i \leq n}\left\{\left(1 / w_{i}\right)\left\|x_{i}\right\|_{\mathcal{H}_{i}}\right\}\right\} \leq 1$ (see [26, Remark 1]), [28, Corollary 2.5] reveals that

$$
\mathrm{P}_{\text {epi } \gamma_{C}}\left(x_{1}, \ldots, x_{n}, \xi\right)= \begin{cases}\left(x_{1}, \ldots, x_{n}, \xi\right), & \text { if } \max _{1 \leq i \leq n}\left\{\frac{1}{w_{i}}\left\|x_{i}\right\|_{\mathcal{H}_{i}}\right\} \leq \xi \\ \left(\bar{y}_{1}, \ldots, \bar{y}_{n}, \bar{\theta}\right), & \text { otherwise }\end{cases}
$$

where

$$
\left(\bar{y}_{1}, \ldots, \bar{y}_{n}\right)=\left(x_{1}, \ldots, x_{n}\right)-\bar{\kappa} \mathrm{P}_{C^{0}}\left(\frac{1}{\bar{\kappa}}\left(x_{1}, \ldots, x_{n}\right)\right), \bar{\theta}=\bar{\kappa}+\xi \text { and } \bar{\kappa}>0 .
$$

By [29, Lemma 4.5] the polar set of $C$ looks like $C^{0}=\left\{\left(x_{1}, \ldots, x_{n}\right) \in \mathcal{H}_{1} \times \ldots \times \mathcal{H}_{n}\right.$ : $\left.\sum_{i=1}^{n} w_{i}\left\|x_{i}\right\|_{\mathcal{H}_{i}} \leq 1\right\}$ and from [28, Lemma 1.1] we derive that $\mathrm{P}_{C^{0}}\left(\frac{1}{\bar{\kappa}}\left(x_{1}, \ldots, x_{n}\right)\right)=$ $\left(x_{1}, \ldots, x_{n}\right)$ if $\sum_{i=1}^{n} w_{i}\left\|x_{i}\right\|_{\mathcal{H}_{i}} \leq \bar{\kappa}$, i.e. $\left(\bar{y}_{1}, \ldots, \bar{y}_{n}\right)=\left(0_{\mathcal{H}}, \ldots, 0_{\mathcal{H}}\right)$, which implies that $\max _{1 \leq i \leq n}\left\{\bar{y}_{i}\right\}=0=\bar{\theta}=\bar{\kappa}+\xi$ and hence, $\bar{\kappa}=-\xi$. 
Otherwise, one has by [28, Lemma 1.1]

$$
\mathrm{P}_{C^{0}}\left(\frac{1}{\bar{\kappa}}\left(x_{1}, \ldots, x_{n}\right)\right)=\left(\bar{z}_{1}, \ldots, \bar{z}_{n}\right) \in \mathcal{H}_{1} \times \ldots \times \mathcal{H}_{n},
$$

where

$$
\bar{z}_{i}=\frac{\max \left\{\left\|x_{i}\right\|_{\mathcal{H}_{i}}-\overline{\kappa \mu} w_{i}, 0\right\}}{\bar{\kappa}\left\|x_{i}\right\|_{\mathcal{H}_{i}}} x_{i}, i=1, \ldots, n,
$$

and $\bar{\mu}>0$ is a solution of the equation [see (11) of the proof of [28, Lemma 1.1]]

$$
\sum_{i=1}^{n} w_{i} \max \left\{\left\|x_{i}\right\|_{\mathcal{H}_{i}}-\overline{\kappa \mu} w_{i}, 0\right\}=\bar{\kappa}
$$

Therefore, it follows

$$
\bar{y}_{i}=x_{i}-\frac{\max \left\{\left\|x_{i}\right\|_{\mathcal{H}_{i}}-\overline{\kappa \mu} w_{i}, 0\right\}}{\left\|x_{i}\right\|_{\mathcal{H}_{i}}} x_{i}=\frac{\left\|x_{i}\right\|_{\mathcal{H}_{i}}-\max \left\{\left\|x_{i}\right\|_{\mathcal{H}_{i}}-\overline{\kappa \mu} w_{i}, 0\right\}}{\left\|x_{i}\right\|_{\mathcal{H}_{i}}} x_{i}, i=1, \ldots, n,
$$

and as for $\left\|x_{i}\right\|_{\mathcal{H}_{i}}-\overline{\kappa \mu} w_{i} \leq 0$ one gets $\bar{y}_{i}=x_{i}$, i.e. $\left\|\bar{y}_{i}\right\|_{\mathcal{H}_{i}}=\left\|x_{i}\right\|_{\mathcal{H}_{i}}$ and for $\left\|x_{i}\right\|_{\mathcal{H}_{i}}-$ $\overline{\kappa \mu} w_{i}>0, \bar{y}_{i}=\left(\overline{\kappa \mu} w_{i} /\left\|x_{i}\right\|_{\mathcal{H}_{i}}\right) x_{i}$, i.e. $\left\|\bar{y}_{i}\right\|_{\mathcal{H}_{i}}=\overline{\kappa \mu} w_{i}, i=1, \ldots, n$, we obtain

$$
\gamma_{C}\left(\bar{y}_{1}, \ldots, \bar{y}_{n}\right)=\max _{1 \leq i \leq n}\left\{\frac{1}{w_{i}}\left\|\bar{y}_{i}\right\|_{\mathcal{H}_{i}}\right\}=\overline{\kappa \mu}=\bar{\kappa}+\xi .
$$

Bringing (34) and (35) together yields

$$
\sum_{i=1}^{n} w_{i} \max \left\{\left\|x_{i}\right\|_{\mathcal{H}_{i}}-(\bar{\kappa}+\xi) w_{i}, 0\right\}=\bar{\kappa} .
$$

Clearly, if $\left\|x_{i}\right\|_{\mathcal{H}_{i}}-\xi w_{i} \leq 0$ for all $i=1, \ldots, n$, i.e. $\max _{1 \leq i \leq n}\left\{\left\|x_{i}\right\|_{\mathcal{H}_{i}} / w_{i}\right\} \leq \xi$, then $\left\|x_{i}\right\|_{\mathcal{H}_{i}}-\xi w_{i}-\bar{\kappa} w_{i} \leq 0$ for all $i=1, \ldots, n$, and one gets by (36) that

$$
\bar{\kappa}=\sum_{i=1}^{n} w_{i} \max \left\{\left\|x_{i}\right\|_{\mathcal{H}_{i}}-(\bar{\kappa}+\xi) w_{i}, 0\right\}=0,
$$

which means that $\bar{y}_{i}=x_{i}$ for all $i=1, \ldots, n$, and $\bar{\theta}=\xi$.

Now, we define the function $g: \mathbb{R} \rightarrow \mathbb{R}$ by

$$
g(\kappa)=\sum_{i=1}^{n} w_{i}^{2} \max \left\{\tau_{i}-(\kappa+\xi), 0\right\}-\bar{\kappa}
$$

Take note, that there exists $i \in\{1, \ldots, n\}$ such that $\tau_{i}>0$ and so,

$$
g\left(\tau_{n}-\xi\right)=\sum_{i=1}^{n} w_{i}^{2} \max \left\{\tau_{i}-\tau_{n}, 0\right\}-\bar{\kappa}=-\bar{\kappa}<0 .
$$

Moreover, as $g$ is a piecewise linear function, one has, similarly to [28, Corollary 2.1], to find the unique integer $k \in\{0,1, \ldots, n-1\}$ such that $g\left(\tau_{k}-\xi\right) \geq 0$ and $g\left(\tau_{k+1}-\xi\right) \leq 0$. This leads to

$$
\sum_{i=k+1}^{n} w_{i}^{2} \tau_{i}-\xi \sum_{i=k+1}^{n} w_{i}^{2}-\bar{\kappa} \sum_{i=k+1}^{n}\left(w_{i}^{2}+1\right)=0 \Leftrightarrow \bar{\kappa}=\frac{\sum_{i=k+1}^{n} w_{i}^{2} \tau_{i}-\xi \sum_{i=k+1}^{n} w_{i}^{2}}{\sum_{i=k+1}^{n} w_{i}^{2}+1}
$$

and hence, $\bar{\theta}=\bar{\kappa}+\xi=\left(\sum_{i=k+1}^{n} w_{i}^{2} \tau_{i}+\xi\right) /\left(\sum_{i=k+1}^{n} w_{i}^{2}+1\right)$. 
Remark 3.17 In [8] the formula in the previous corollary was given for the case where $\mathcal{H}_{i}=\mathbb{R}$, $i=1, \ldots, n$, in other words, where $\gamma_{C}$ is the weighted $l_{\infty}$-norm.

Remark 3.18 The proof of the previous theorem allows us to construct an algorithm to determine $\bar{\kappa}$ of Theorem 3.9.

\section{Algorithm}

1. If $\max _{1 \leq i \leq n}\left\{\frac{1}{w_{i}}\left\|x_{i}\right\|_{\mathcal{H}_{i}}\right\} \leq \xi$, then $\bar{\kappa}=0$.

2. If $\xi<0$ and $\sum_{i=1}^{n} w_{i}\left\|x_{i}\right\|_{\mathcal{H}_{i}} \leq-\xi$, then $\bar{\kappa}=-\xi$.

3. Otherwise, define $\tau_{0}:=0, \tau_{i}:=\left\|x_{i}\right\|_{\mathcal{H}_{i}} / w_{i}, i=1, \ldots, n$, and sort $\tau_{0}, \ldots, \tau_{n}$ in ascending order.

4. Determine the values of $g$ defined in (37) at $\kappa=\tau_{i}+\xi, i=0, \ldots, n$.

5. Find the unique $k \in\{0, \ldots, n-1\}$ such that $g\left(\tau_{k}-\xi\right) \geq 0$ and $g\left(\tau_{k+1}-\xi\right) \leq 0$.

6. Calculate $\bar{\kappa}$ by (33).

\section{Numerical experiments}

The aim in this section is to solve numerically several types of concrete location problems and their associated duals discussed in the previous section as well as to discuss the results generated via MATLAB. Here we set $X=\mathbb{R}^{d}$ and used for our numerical experiments a PC with an Intel Core i5 $2400 \mathrm{CPU}$ with $3.10 \mathrm{GHz}$ and 8GB RAM. Note that in the previous sections we have considered for the theoretical investigations very general frameworks, however, in order to get closer to the real world applications, our numerical experiments are performed in finitely dimensional spaces.

First, we consider a location problem of the type analyzed in Sect. 3.3. To solve this kind of a location problem and its dual, rewritten as unconstrained optimization problems, we implemented in MATLAB the parallel splitting algorithm presented in [3, Proposition 27.8]. Note also that other recent proximal splitting methods could prove to be suitable for these problems, too, and comparisons of their performances on the problems we solve below could constitute an interesting follow-up of our investigations.

Theorem 4.1 (Parallel splitting algorithm) Let $n$ be an integer such that $n \geq 2$ and $f_{i}$ : $\mathbb{R}^{d} \rightarrow \overline{\mathbb{R}}$ be a proper, lower semicontinuous and convex function for $i=1, \ldots, n$. Suppose that the problem

$$
\left(P^{D R}\right) \min _{x \in \mathbb{R}^{s}}\left\{\sum_{i=1}^{n} f_{i}(x)\right\}
$$

has at least one solution and that $\operatorname{dom} f_{1} \cap \bigcap_{i=2}^{n} \operatorname{int} \operatorname{dom} f_{i} \neq \emptyset$. Let $\left(\mu_{k}\right)_{k \in \mathbb{N}}$ be a sequence in $[0,2]$ such that $\sum_{k \in \mathbb{N}} \mu_{k}\left(2-\mu_{k}\right)=+\infty$, let $v>0$, and let $\left(x_{i, 0}\right)_{i=1}^{n} \in \mathbb{R}^{d} \times \ldots \times \mathbb{R}^{d}$. Set

$$
\begin{array}{l|l}
(\forall k \in \mathbb{N}) & r_{k}=\frac{1}{n} \sum_{i=1}^{n} x_{i, k}, \\
& y_{i, k}=\operatorname{prox}_{v f_{i}} x_{i, k}, i=1, \ldots, n \\
& q_{k}=\frac{1}{n} \sum_{i=1}^{n} y_{i, k}, \\
& x_{i, k+1}=x_{i, k}+\mu_{k}\left(2 q_{k}-r_{k}-y_{i, k}\right), i=1, \ldots, n
\end{array}
$$


Then $\left(r_{k}\right)_{k \in \mathbb{N}}$ converges to an optimal solution to $\left(P^{D R}\right)$.

Take note, that for this purpose it is necessary to bring the location problem and its dual problem into the form of an unconstrained optimization problem where the objective function is a sum of proper, lower semicontinuous and convex functions. By following these ideas, we rewrite the location problem (see Remark 3.7)

$$
\begin{aligned}
& \left(P_{\gamma_{G}}, \mathcal{T}\right) \inf _{x \in X} \max _{1 \leq i \leq n}\left\{\mathcal{T}_{\Omega_{i}, \gamma_{G_{i}}}^{C_{i}}(x)\right\}=\min _{\substack{t>0, x \in \mathbb{R}^{d}, z_{i} \in \mathbb{R}^{d}, \gamma_{C_{i}}\left(x-p_{i}-z_{i}\right)+\gamma_{G_{i}}\left(z_{i}\right) \leq t, i=1, \ldots, n}} t \\
& =\min _{\substack{t>0, x \in \mathbb{R}^{d}, z_{i} \in \mathbb{R}^{d}, \alpha_{i} \geq 0, \beta_{i} \geq 0, \gamma_{C_{i}}\left(x-p_{i}-z_{i}\right) \leq \alpha_{i}, \gamma_{G_{i}}\left(z_{i}\right) \leq \beta_{i}, \alpha_{i}+\beta_{i}=t, i=1, \ldots, n}} t=\min _{\begin{array}{c}
t>0, x \in \mathbb{R}^{d}, z_{i} \in \mathbb{R}^{d}, \alpha_{i} \geq 0, \beta_{i} \geq 0, \\
\left(x-p_{i}-z_{i}, \alpha_{i}\right) \in \text { epi } \gamma_{C_{i}},\left(z_{i}, \beta_{i}\right) \in \text { epi } \gamma_{G_{i}}, \\
\alpha_{i}+\beta_{i}=t, i=1, \ldots, n
\end{array}} t,
\end{aligned}
$$

where $C_{i}$ and $G_{i}$ are closed and convex subsets of $\mathbb{R}^{d}$ with $0_{\mathbb{R}^{d}} \in \operatorname{int} C_{i}, 0_{\mathbb{R}^{d}} \in \operatorname{int} G_{i}$, and $\Omega_{i}=\left\{p_{i}\right\}$ with $p_{i} \in \mathbb{R}^{d}, i=1, \ldots, n$, as follows

$$
\begin{aligned}
& \left(P_{\gamma_{G}, \mathcal{T}}\right) \min _{\substack{t>0, \alpha_{i} \geq 0, \mathbb{R}^{d}, \beta_{i} \geq 0, i=1, \ldots, n}}\left\{t+\sum_{i=1}^{n}\left[\delta_{\text {epi } \gamma_{C_{i}}}\left(x-p_{i}-z_{i}, \alpha_{i}\right)\right.\right. \\
& \left.\left.+\delta_{\text {epi } \gamma_{G_{i}}}\left(z_{i}, \beta_{i}\right)\right]+\delta_{H}(\alpha, \beta, t)\right\} \text {, }
\end{aligned}
$$

where $\alpha=\left(\alpha_{1}, \ldots, \alpha_{n}\right)^{\top}, \beta=\left(\beta_{1}, \ldots, \beta_{n}\right)^{\top}$ and $H=\left\{(\alpha, \beta, t)^{\top}: \alpha_{i}+\beta_{i}=t, i=\right.$ $1, \ldots, n\}$. Similarly, the dual problem

$$
\begin{aligned}
& \left(D_{\gamma_{G}}, \mathcal{T}\right) \quad \max _{z_{i}^{*} \geq 0, w_{i}^{*} \in \mathbb{R}^{d}, \gamma_{C_{i}^{0}}\left(w_{i}^{*}\right) \leq z_{i}^{*},} \quad\left\{-\sum_{i \in I} \sigma_{\Omega_{i}}\left(w_{i}^{*}\right)\right\}, \\
& \gamma_{G_{i}^{0}}\left(w_{i}^{*}\right) \leq z_{i}^{*}, i=1, \ldots, n, \sum_{i=1}^{n} z_{i}^{*} \leq 1, \sum_{i=1}^{n} w_{i}^{*}=0_{\mathbb{R}^{d}}
\end{aligned}
$$

can be equivalently rewritten as

$$
\begin{gathered}
\left(D_{\gamma_{G}, \mathcal{T}}\right)-\min _{\substack{z_{i}^{*} \geq 0, w_{i}^{*} \in \mathbb{R}^{d} \\
i=1, \ldots, n}}\left\{\sum_{i=1}^{n}\left[p_{i}^{\top} w_{i}^{*}+\delta_{\mathrm{epi} \gamma_{C_{i}^{0}}}\left(w_{i}^{*}, z_{i}^{*}\right)+\delta_{\mathrm{epi} \gamma_{G_{i}^{0}}}\left(w_{i}^{*}, z_{i}^{*}\right)\right]\right. \\
\left.+\delta_{D}\left(z^{*}\right)+\delta_{E}\left(w^{*}\right)\right\},
\end{gathered}
$$

where $z^{*}=\left(z_{1}^{*}, \ldots, z_{n}^{*}\right)^{\top}, w^{*}=\left(w_{1}^{*}, \ldots, w_{n}^{*}\right), D=\left\{z^{*} \in \mathbb{R}_{+}^{n}: \sum_{i=1}^{n} z_{i}^{*} \leq 1\right\}$ and $E=\left\{w^{*} \in \mathbb{R}^{d} \times \ldots \times \mathbb{R}^{d}: \sum_{i=1}^{n} w_{i}^{*}=0_{\mathbb{R}^{d}}\right\}$. For both these optimization problems the nonnegativity constraints can be omitted because they are implicitly contained in the indicator functions of the epigraphs of gauges from the objective functions and one can verify that the hypotheses of Theorem 4.1 are fulfilled. Moreover, for the full implementation of this algorithm for solving these problems numerically, one requires also formulae of the proximal mappings of the functions involved in the objective function of the primal and the dual problem, which can be found for instance in [3,28].

Example 4.1 Take $\gamma_{C_{i}}=\|\cdot\|$ and $\gamma_{G_{i}}=\|\cdot\|_{\infty}, i=1, \ldots, 5$, and set $d=2, \Omega_{i}=\left\{p_{i}\right\}$, $i=1, \ldots, 5$, with $p_{1}=(-8,-9)^{T}, p_{2}=(10,0)^{T}, p_{3}=(11,5)^{T}, p_{4}=(-12,10)^{T}$ and $p_{5}=(4,13)^{T}$. To compute the required proximal and projection points in the primal 
Table 1 Performance evaluation for 5 points in $\mathbb{R}^{2}$

\begin{tabular}{llllll}
\hline & \multicolumn{1}{c}{$\varepsilon_{1}=10^{-4}$} & & & $\varepsilon_{2}=10^{-8}$ & \\
\cline { 2 - 3 } & Primal $(v=6)$ & Dual $(v=0.08)$ & & Primal $(v=6)$ & Dual $(v=0.08)$ \\
\hline CPU time (s) & 0.2889 & 0.2049 & 0.7196 & 0.6352 \\
Number of iterations & 749 & 783 & 1949 & 2446 \\
\hline
\end{tabular}

and dual problem we used Theorem 3.9 , [28, Lemma 2.1], [28, Lemma 2.2], [28, Corollary 2.1], [3, Proposition 23.32] and [3, Example 28.14 (iii)]. We ran our MATLAB programs for various step sizes $v$ and chose always the origin as the starting point and set the initialization parameters to the value 1 . The best performance results of our tests are illustrated in Table 1. As stopping criterion for the iteration of both programs we used the values $\varepsilon_{1}=10^{-4}$ and $\varepsilon_{2}=$ $10^{-8}$, which define the maximum bounds from the optimal solution, respectively. MATLAB computed for the location problem (primal) an optimal location at $\bar{x}=(-0.5,2.2878)^{T}$ with

$$
\begin{aligned}
& \bar{z}_{1}=(5.9851,5.9851)^{T}, \bar{z}_{2}=(-3.3359,3.0337)^{T}, \bar{z}_{3}=(-3.7516,-2.7122)^{T}, \\
& \bar{z}_{4}=(7.7122,-7.7122)^{T}, \bar{z}_{5}=(-3.4694,-3.4402)^{T}, \\
& \left(\bar{\alpha}_{1}, \ldots, \bar{\alpha}_{5}\right)^{T}=(5.5149,7.6765,7.7484,3.7878,7.6526)^{T} \\
& \left(\bar{\beta}_{1}, \ldots, \bar{\beta}_{5}\right)^{T}=(5.9851,3.8235,3.7516,7.7122,3.8474)^{T},
\end{aligned}
$$

where the optimal objective value was $v\left(P_{\gamma_{G}}, \mathcal{T}\right)=11.5$. Note that the optimal solution of the location problem is not unique and may differ for each chosen step size and starting point.

For the dual problem the following optimal solution was computed $\bar{w}_{1}^{*}=\bar{w}_{2}^{*}=\bar{w}_{5}^{*}=$ $(0,0)^{T}, \bar{w}_{3}^{*}=(-0.5,0)^{T}$ and $\bar{w}_{4}^{*}=(0.5,0)^{T}$ with the objective function value $v\left(D_{\gamma_{G}}, \mathcal{T}\right)=$ 11.5 , i.e. $v\left(P_{\gamma_{G}}, \mathcal{T}\right)=v\left(D_{\gamma_{G}}, \mathcal{T}\right)$. Note that, similar to Remark 3.16, one can understand the vectors $\bar{w}_{i}^{*}, i=1, \ldots, n$, as force vectors fulfilling the optimality conditions of Theorem 3.4 and increasing the maximum norm balls centered at the given points and the Euclidean norm balls centered at $\bar{x}$ until their intersection is non-empty. Especially, it follows from the optimality conditions (iv) and (vi) that an index $i$ belongs to the optimal index set $\bar{I}$, if the value of the associated extended minimal time function $\mathcal{T}_{\Omega_{i}, \gamma_{G_{i}}}^{C_{i}}$ at $\bar{x}$ is equal to $\bar{\lambda}$, which is exactly the case when the corresponding vector $\bar{w}_{i}^{*}$ is unequal to the zero vector (in our example $\bar{I}=\{3,4\}$, see Fig. 1). At this point it is also important to say, that for a better visualization we multiplied the vectors, characterizing the optimal solution of the dual problem, in all figures with the value 3 .

One can note in Table 1 that the primal method needs less iterations, while the dual method generates faster a solution which is within the maximum bound from the optimal solution. By using the formula from Remark 3.6, the optimal location can be determined immediately:

$$
\bar{x}=\frac{1}{0.5+0.5}\left(0.5 \cdot(11,5)^{T}+0.5 \cdot(-12,10)^{T}\right)=(-0.5,7.5)^{T} .
$$

We also considered primal and dual problems defined by 20 given points. The computational information can be seen in Table 2 and draws a similar picture as in the previous situation. If we increase the accuracy to $\varepsilon_{2}=10^{-8}$ the dual method is faster than the primal method, which could be especially a benefit for location problems with a large number of given points. 


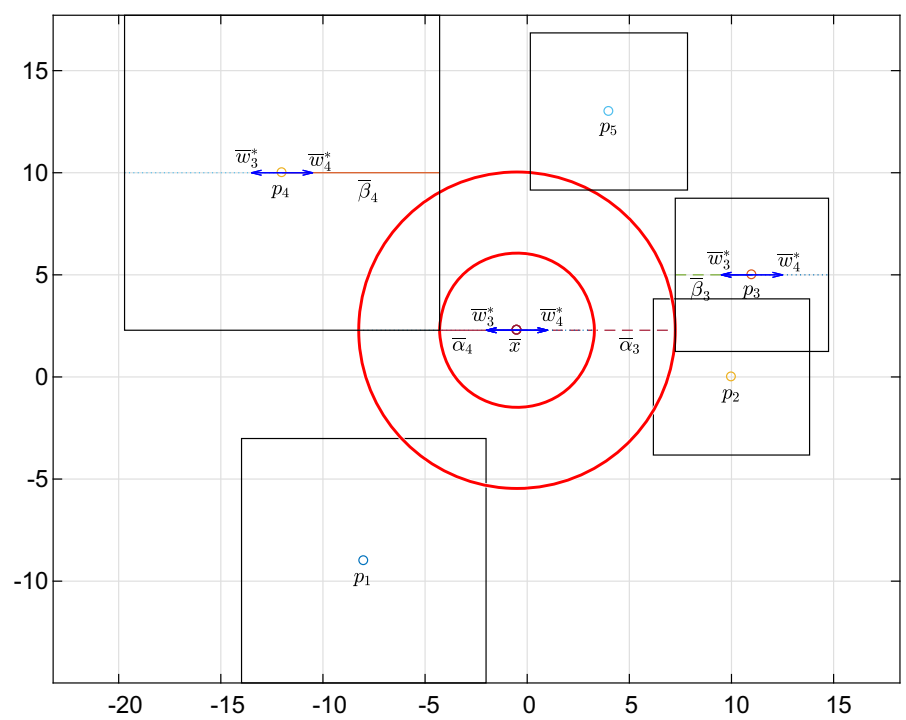

Fig. 1 Visualization of the optimal solutions of the location problem $\left(P_{\gamma_{G}}, \mathcal{T}\right)$ and its dual problem $\left(D_{\gamma_{G}}, \mathcal{T}\right)$

Table 2 Performance evaluation for 20 points in $\mathbb{R}^{2}$

\begin{tabular}{llllll}
\hline & \multicolumn{1}{l}{$\varepsilon_{1}=10^{-4}$} & & $\varepsilon_{2}=10^{-8}$ & \\
\cline { 2 - 3 } \cline { 5 - 6 } & Primal $(v=6)$ & Dual $(v=0.08)$ & & Primal $(v=6)$ & Dual $(v=0.08)$ \\
\hline CPU time (s) & 6.6447 & 12.6774 & 85.0740 & 68.3818 \\
Number of iterations & 3802 & 13,155 & 29,334 & 36,604 \\
\hline
\end{tabular}

The second scenario of our numerical approach relies on the location problems discussed in Sect. 3.3. In this situation the location problem can be rewritten as follows

$$
\begin{gathered}
\left(P_{\mathcal{T}}\right) \min _{x \in \mathbb{R}^{d}} \max _{1 \leq i \leq n}\left\{\mathcal{T}_{\Omega_{i}, \delta_{L_{i}}}^{C_{i}}(x)\right\}=\min _{\substack{t \geq 0, x \in \mathcal{H}, y_{i} \in \Omega_{i}, z_{i} \in L_{i} \\
i=1, \ldots, n}} \min _{\left.C_{i}\left(x-y_{i}-z_{i}\right)\right\} \leq t,} t=\min _{\substack{t \geq 0, x \in \mathbb{R}^{d}, y_{i} \in \Omega_{i}, z_{i} \in L_{i},\left(x-y_{i}-z_{i}, t\right) \in \operatorname{epi} \gamma_{C_{i}}, i=1, \ldots, n}} \\
t=\min _{\substack{t \geq 0, x, y_{i}, z_{i} \in \mathbb{R}^{d} \\
i=1, \ldots, n}}\left\{t+\sum_{i=1}^{n}\left[\delta_{\mathrm{epi} \gamma_{C_{i}}}\left(x-y_{i}-z_{i}, t\right)+\delta_{\Omega_{i}}\left(y_{i}\right)+\delta_{L_{i}}\left(z_{i}\right)\right]\right\},
\end{gathered}
$$

where $C_{i}, L_{i} \subseteq \mathbb{R}^{d}$ are closed and convex sets with $0_{\mathbb{R}^{d}} \in$ int $C_{i}$ and $\Omega_{i} \subseteq \mathbb{R}^{d}$ are convex and compact sets, $i=1, \ldots, n$, and likewise one gets for its dual problem

$$
\begin{aligned}
& \left(\widetilde{D}_{\mathcal{T}}\right) \max _{\substack{y_{i}^{*} \in \mathbb{R}^{d}, i=1, \ldots, n, \sum_{i=1}^{n} \gamma_{C_{i}^{0}}\left(y_{i}^{*}\right) \leq 1, \sum_{i=1}^{n} y_{i}^{*}=0_{\mathbb{R}^{d}}}}\left\{-\sum_{i=1}^{n}\left[\sigma_{L_{i}}\left(y_{i}^{*}\right)+\sigma_{\Omega_{i}}\left(y_{i}^{*}\right)\right]\right\} \\
& =-\min _{y_{i}^{*} \in \mathbb{R}^{d}, i=1, \ldots, n}\left\{\sum_{i=1}^{n}\left[\sigma_{L_{i}}\left(y_{i}^{*}\right)+\sigma_{\Omega_{i}}\left(y_{i}^{*}\right)\right]+\delta_{F}\left(y_{i}^{*}\right)+\delta_{E}\left(y_{i}^{*}\right)\right\},
\end{aligned}
$$


Table 3 Performance evaluation for 7 points in $\mathbb{R}^{2}$

\begin{tabular}{|c|c|c|c|c|}
\hline \multirow{2}{*}{+2} & \multicolumn{2}{|l|}{$\varepsilon_{1}=10^{-4}$} & \multicolumn{2}{|l|}{$\varepsilon_{2}=10^{-8}$} \\
\hline & Primal $(v=39)$ & Dual $(v=0.11)$ & Primal $(v=39)$ & Dual $(v=0.11)$ \\
\hline CPU time (s) & 0.3786 & 0.1174 & 0.7640 & 0.2973 \\
\hline Number of iterations & 541 & 330 & 1106 & 830 \\
\hline
\end{tabular}

where $y^{*}=\left(y_{1}^{*}, \ldots, y_{n}^{*}\right), E=\left\{y^{*} \in \mathbb{R}^{d} \times \ldots \times \mathbb{R}^{d}: \sum_{i=1}^{n} y_{i}^{*}=0_{\mathbb{R}^{d}}\right\}$ and $F=\left\{y^{*} \in\right.$ $\left.\mathbb{R}^{d} \times \ldots \times \mathbb{R}^{d}: \sum_{i=1}^{n} \gamma_{C_{i}^{0}}\left(y_{i}^{*}\right) \leq 1\right\}$. The nonnegativity constraint of $\left(P_{\mathcal{T}}\right)$ can be omitted because it is implicitly contained in some indicator functions from the objective functions and one can then verify that the hypotheses of Theorem 4.1 are fulfilled.

Example 4.2 Let $d=2, \quad p_{1}=(-8,8)^{T}, \quad p_{2}=(-7,0)^{T}, p_{3}=(-4,-1)^{T}, p_{4}=$ $(2,0)^{T}, p_{5}=(2,-6)^{T}, p_{6}=(7,1)^{T}, p_{7}=(6,5)^{T}, a_{1}=1, a_{2}=2, a_{3}=3, a_{4}=$ $0.5, a_{5}=2, a_{6}=1, a_{7}=1, b_{1}=0.5, b_{2}=2, b_{3}=0.6, b_{4}=1, b_{5}=1.5, b_{6}=$ $1, b_{7}=0.5, \Omega_{i}=\left\{x \in \mathbb{R}^{2}:\left\|x-p_{i}\right\|_{\infty} \leq a_{i}\right\}, L_{i}=\left\{x \in \mathbb{R}^{2}:\|x\| \leq b_{i}\right\}$ and $\gamma_{C_{i}}=\|\cdot\|$, $i=1, \ldots, 7$. Note that in this case $\sigma_{L_{i}}=\|\cdot\|$ and $\sigma_{\Omega_{i}}=\|\cdot\|_{1}, i=1, \ldots, 7$. Using the formulae given in [28, Corollary 2.3], [28, Corollary 2.1] and [28, Lemma 1.1] to compute the proximal and projection points regarding the location problem and its dual, we tested various step sizes $v$, where the starting point was always the origin and the initialization parameters were set to the value 1 . The best performance results are presented in Table 3 and visualized in Fig. 2 (note, that for a better visualization we multiplied the vectors, characterizing the optimal solution of the dual problem, with the value 3 ). The cancellation criterion for ending the iteration for both programs were the values $\varepsilon_{1}=10^{-4}$ and $\varepsilon_{2}=10^{-8}$, the maximum bounds from the optimal solution. The optimal location we obtained is $\bar{x}=(-1.0765,3.7039)^{T}$ and the optimal objective value is $v\left(P_{\mathcal{T}}\right)=6.2788$. Let us remark that the optimal solution of the location problem is not unique and may differ for each chosen step size. The optimal solution of the dual problem was found at $\bar{y}_{1}^{*}=(0.4072,-0.2266)^{T}, \bar{y}_{2}^{*}=\bar{y}_{3}^{*}=\bar{y}_{4}^{*}=(0,0)^{T}$, $\bar{y}_{5}^{*}=(-0.0186,0.1330)^{T}, \bar{y}_{6}^{*}=(-0.3886,0.0936)^{T}$ and $\bar{y}_{7}^{*}=(0,0)^{T}$, while the objective function value was $v\left(\widetilde{D}_{\mathcal{T}}\right)=6.2788$, which means that $v\left(P_{\mathcal{T}}\right)=v\left(\widetilde{D}_{\mathcal{T}}\right)$. In Table 3 one can note that the dual method needed less CPU time as well as fewer iterations to determine a solution which is within the maximum bound from the optimal solution compared to the method which solves the location problem directly. The optimal location can be reconstructed by using the formulae given in Remark 3.15.

Setting $L_{i}=\left\{0_{\mathbb{R}^{d}}\right\}, i=1, \ldots, n$, we were able to compare our two methods with the one presented in [17, Theorem 4.1] (or [13, Theorem 4.69]) that employs the subgradient method for solving the following generalized Sylvester problem

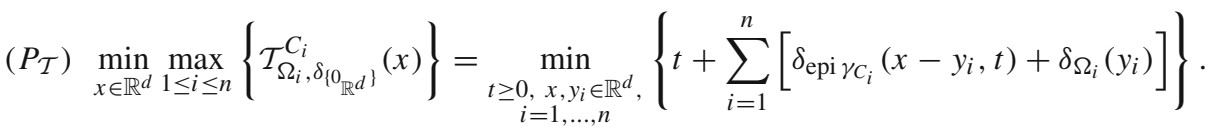

The corresponding dual problem looks then as follows

$$
\left(\widetilde{D}_{\mathcal{T}} \max _{\substack{y_{i}^{*} \in \mathbb{R}^{d}, i=1, \ldots, n, \sum_{i=1}^{n} \gamma_{C_{i}^{0}}\left(y_{i}^{*}\right) \leq 1, \sum_{i=1}^{n} y_{i}^{*}=0_{\mathbb{R}^{d}}}}\left\{-\sum_{i=1}^{n} \sigma_{\Omega_{i}}\left(y_{i}^{*}\right)\right\}\right.
$$




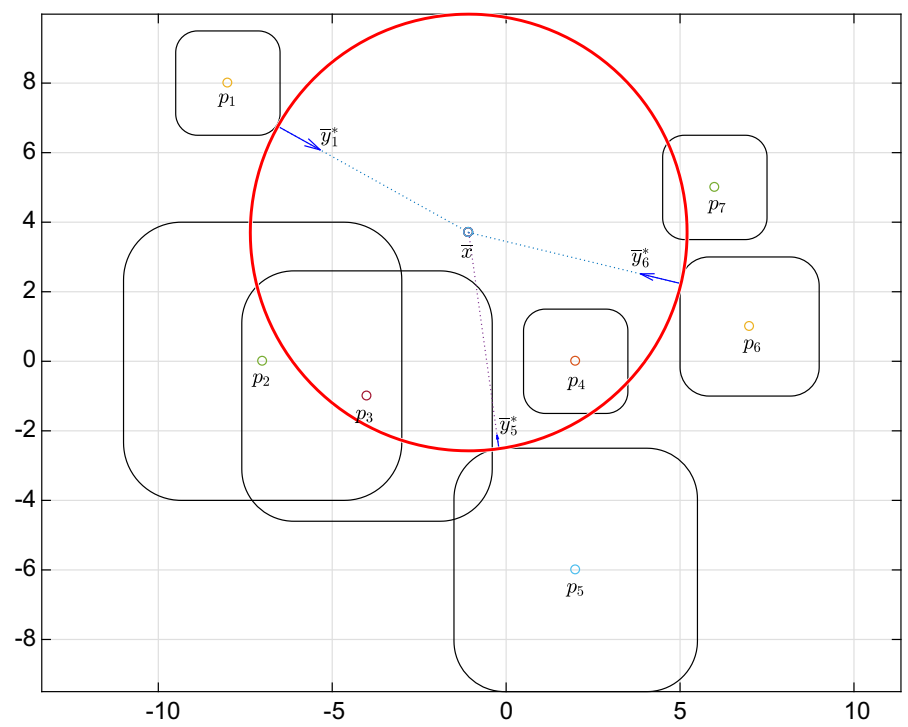

Fig. 2 Visualization of the optimal solutions of the location problem $\left(P_{\mathcal{T}}\right)$ and its dual problem $\left(\widetilde{D}_{\mathcal{T}}\right)$

$$
=-\min _{\substack{y_{i}^{*} \in \mathbb{R}^{d}, i=1, \ldots, n}}\left\{\sum_{i=1}^{n} \sigma_{\Omega_{i}}\left(y^{*}\right)+\delta_{F}\left(y^{*}\right)+\delta_{E}\left(y^{*}\right)\right\} .
$$

Theorem 4.2 (cf. [13, Theorem 4.69]) Let $\mathcal{H}=\mathbb{R}^{m}$, fix $x_{1} \in \mathbb{R}^{m}$ and define the sequences of iterates by

$$
x_{k+1}:=x_{k}-\alpha_{k} v_{k}, k \in \mathbb{N},
$$

where $\left\{\alpha_{k}\right\}$ are positive numbers, and where

$$
v_{k} \in \begin{cases}\left\{0_{\mathbb{R}^{m}}\right\}, & \text { if } x_{k} \in \Omega_{i}+L_{i}, \\ {\left[-\partial\|\cdot\|\left(w_{k}-x_{k}\right)\right] \cap N_{\Omega_{i}+L_{i}}\left(w_{k}\right),} & \text { if } x_{k} \notin \Omega_{i}+L_{i},\end{cases}
$$

where $w_{k}=\mathrm{P}_{\Omega_{i}+L_{i}}\left(x_{k}\right)$ for some $i \in I=\left\{j=1, \ldots, n: \mathcal{T}_{\Omega_{j}, \delta_{L_{j}}}^{C_{j}}(x)=\max _{1 \leq l \leq n}\left\{\mathcal{T}_{\Omega_{l}, \delta_{L_{l}}}^{C_{l}}(x)\right\}\right\}$.

Define the value sequence

$$
V_{k}:=\min \left\{\max _{1 \leq l \leq n}\left\{\mathcal{T}_{\Omega_{l}, \delta_{L_{l}}}^{C_{l}}(x)\right\}\right\} .
$$

If the sequence $\left\{\alpha_{k}\right\}$ satisfies $\sum_{k=1}^{\infty} \alpha_{k}=\infty$ and $\sum_{k=1}^{\infty} \alpha_{k}^{2}<\infty$, then $\left\{V_{k}\right\}$ converges to the optimal value $\bar{V}$ and $\left\{x_{k}\right\}$ converges to an optimal solution $\bar{x}$ to the problem $\left(P_{\mathcal{T}}\right)$.

Example 4.3 (cf. [17, Example 4.3]) Let $d=2, p_{1}=(-8,8)^{T}, p_{2}=(-7,0)^{T}, p_{3}=$ $(-4,-1)^{T}, p_{4}=(2,0)^{T}, p_{5}=(2,-6)^{T}, p_{6}=(7,1)^{T}, p_{7}=(6,5)^{T}, a_{1}=1, a_{2}=$ $2, a_{3}=3, a_{4}=0.5, a_{5}=2, a_{6}=1, a_{7}=1, \Omega_{i}=\left\{x \in \mathbb{R}^{2}:\left\|x-p_{i}\right\|_{\infty} \leq a_{i}\right\}, L_{i}=$ $\left\{0_{\mathbb{R}^{2}}\right\}, \gamma_{C_{i}}=\|\cdot\|, \quad i=1, \ldots, 7$. We tested the subgradient method for the sequence $\alpha_{k_{1}}=1 / k$, which also was used in [17], as well as for the sequence $\alpha_{k_{1}}=1 / \sqrt{k+1}$ (see [20, Section 3.2.3]). Note that the subgradient method is simple to implement and 
can be employed for solving minmax location problems generated by various norms and generalized distances. The algorithms considered in this paper seem at the first look to be more complicated, also due to the necessity of determining some epigraphical projections, however, as seen below, they work faster and cheaper, and, taking for instance the results from Sect. 3, they can be employed for solving minmax location problems generated by various norms and generalized distances as well.

For our numerical experiments we again used for all programs as starting point the origin and for the cancellation criterion for ending the iteration the values $\varepsilon_{1}=10^{-4}$ and $\varepsilon_{2}=10^{-8}$. Especially, we set again in the parallel splitting algorithms the initialization parameters to the value 1 and tested these methods for various step sizes $v$. The best performance results were reached for the step sizes $v=24$ and $v=0.076$ for the location problem (primal) and its associated dual problem, respectively. The optimal location was found to be $\bar{x}=$ $(-1.0556,3.0556)^{T}$ and for the optimal objective value we got the value $v\left(P_{\mathcal{T}}\right)=7.1340$. The optimal solution of the dual problem was determined as $\bar{y}_{1}^{*}=(0.3755,-0.2491)^{T}, \bar{y}_{2}^{*}=$ $\bar{y}_{3}^{*}=\bar{y}_{4}^{*}=\bar{y}_{7}^{*}=\left(\widetilde{\sim}_{\mathcal{T}}, 0\right)^{T}, \bar{y}_{5}^{*}=(-0.0295,0.1974)^{T}, \bar{y}_{6}^{*}=(-0.3459,0.0518)^{T}$ and the objective value $v\left(\widetilde{D}_{\mathcal{T}}\right)=7.1340$, i.e. $v\left(P_{\mathcal{T}}\right)=v\left(\widetilde{D}_{\mathcal{T}}\right)$ (see Fig. 3 ). As the Tables 4 and 5 demonstrate, the dual method performs once again very well, especially for the accuracy $\varepsilon_{2}=10^{-8}$. While the subgradient method is the fastest one for the accuracy $\varepsilon_{1}=10^{-4}$ and the sequence $\alpha_{k_{1}}=1 / k$, it has not reached the precision $\varepsilon_{2}=10^{-8}$ after passing 500,000 iterations.

In Tables 6 and 7 we present the computational results obtained while solving a location problem defined by 50 points, where the dual method performs once again very well and is faster as the primal method. For the sequence $\alpha_{k_{1}}=1 / k$ the subgradient method has not reached machine precision after passing 500,000 iterations, whereas the sequence $\alpha_{k_{2}}=$ $1 / \sqrt{k+1}$ performs surprisingly very well for $\varepsilon_{2}=10^{-8}$. Hence, for the accuracy $\varepsilon_{2}=10^{-8}$ the sequence $\alpha_{k_{2}}=1 / \sqrt{k+1}$ is the optimal strategy for the subgradient method, as it also

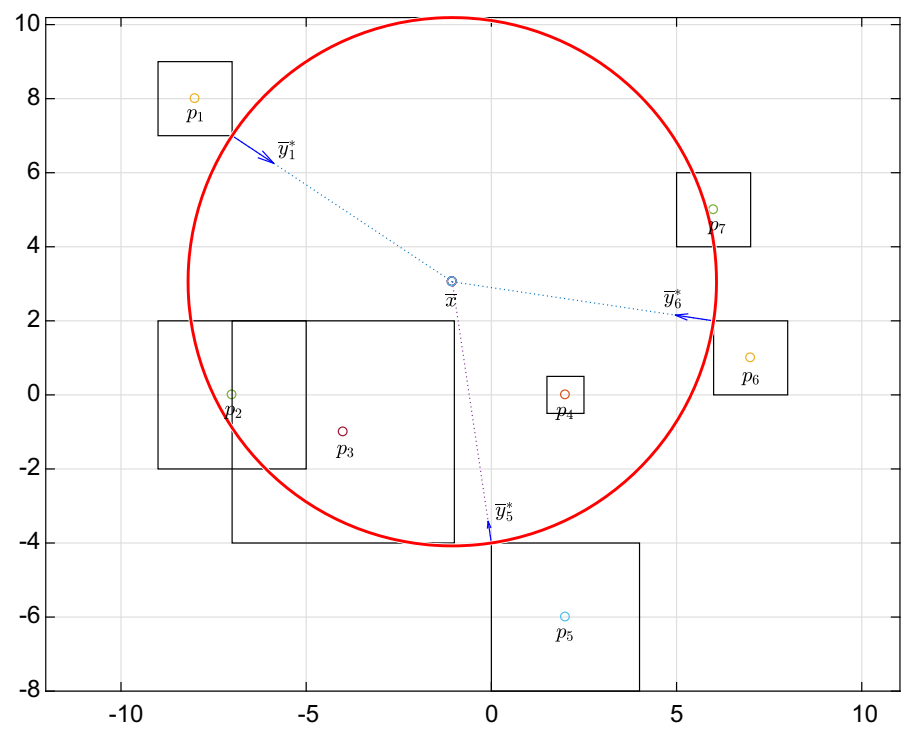

Fig. 3 Visualization of the optimal solutions of the location problem $\left(P_{\mathcal{T}}\right)$ and its dual problem $\left(\widetilde{D}_{\mathcal{T}}\right)$ 
Table 4 Performance evaluation for 7 points in $\mathbb{R}^{2}$ with $\varepsilon_{1}=10^{-4}$

\begin{tabular}{lllll}
\hline & Primal $(v=24)$ & Dual $(v=0.076)$ & Subgrad. $\left(\alpha_{k_{1}}\right)$ & Subgrad. $\left(\alpha_{k_{2}}\right)$ \\
\hline CPU time (s) & 0.1904 & 0.0871 & 0.0416 & 1.2782 \\
Number of iteration & 399 & 181 & 918 & 70,752 \\
\hline
\end{tabular}

Table 5 Performance evaluation for 7 points in $\mathbb{R}^{2}$ with $\varepsilon_{2}=10^{-8}$

\begin{tabular}{lllll}
\hline & Primal $(v=24)$ & Dual $(v=0.076)$ & Subgrad. $\left(\alpha_{k_{1}}\right)$ & Subgrad. $\left(\alpha_{k_{2}}\right)$ \\
\hline CPU time (s) & 0.3377 & 0.1608 & 0.7016 & - \\
Number of iterations & 730 & 453 & 37,854 & $>500,000$ \\
\hline
\end{tabular}

Table 6 Performance evaluation for 50 points in $\mathbb{R}^{2}$ with $\varepsilon_{1}=10^{-4}$

\begin{tabular}{lllll}
\hline & Primal $(v=27)$ & Dual $(v=0.025)$ & Subgrad. $\left(\alpha_{k_{1}}\right)$ & Subgrad. $\left(\alpha_{k_{2}}\right)$ \\
\hline CPU time (s) & 5.6477 & 0.4292 & - & 27.1555 \\
Number of iterations & 2421 & 735 & $>500,000$ & 383,782 \\
\hline
\end{tabular}

Table 7 Performance evaluation for 50 points in $\mathbb{R}^{2}$ with $\varepsilon_{2}=10^{-8}$

\begin{tabular}{lllll}
\hline & Primal $(v=27)$ & Dual $(v=0.0046)$ & Subgrad. $\left(\alpha_{k_{1}}\right)$ & Subgrad. $\left(\alpha_{k_{2}}\right)$ \\
\hline CPU time $(\mathrm{s})$ & 16.1011 & 3.6020 & - & 32.2530 \\
Number of iterations & 6983 & 7207 & - & 436,138 \\
\hline
\end{tabular}

was shown in [20, Section 3.2.3] under the additional assumption that the objective function is Lipschitz continuous.

In the next, we present an example in the three-dimensional space, where we compared the two parallel splitting algorithms for the location problem and its dual, respectively.

Example 4.4 Let $d=3, p_{1}=(-8,8,8)^{T}, p_{2}=(-7,0,0)^{T}, p_{3}=(-4,-1,1)^{T}, p_{4}=$ $(2,0,2)^{T}, \quad p_{5}=(2,-6,2)^{T}, p_{6}=(7,1,1)^{T}, p_{7}=(6,5,4)^{T}, a_{1}=\ldots=a_{7}=$ $0.5, \Omega_{i}=\left\{x \in \mathbb{R}^{3}:\left\|x-p_{i}\right\|_{\infty} \leq a_{i}\right\}, L_{i}=\left\{0_{\mathbb{R}^{3}}\right\}, \gamma_{C_{i}}=\|\cdot\|, i=1, \ldots, 7$. For the numerical tests we used here the same values for the initialization parameters, starting point and stopping criterion for the iteration as in the previous example. The performance results were determined for the step sizes $v=10$ and $v=0.055$ for the location problem and its associated dual problem, respectively, and are presented in Table 8. The optimal location was identified at $\bar{x}=(-1.4350,2.2492,4.5693)^{T}$ and for the optimal objective value we got the value $v\left(P_{\mathcal{T}}\right)=8.5408$. The optimal solution of the dual problem was determined as $\bar{y}_{1}^{*}=(0.3289,-0.2848,-0.1589)^{T}, \bar{y}_{2}^{*}=\bar{y}_{3}^{*}=\bar{y}_{4}^{*}=\bar{y}_{7}^{*}=(0,0,0)^{T}$, $\bar{y}_{5}^{*}=(-0.0997,0.2632,0.0703)^{T}$ and $\bar{y}_{6}^{*}=(-0.2292,0.0216,0.0887)^{T}$ and the objective value $v\left(D_{\mathcal{T}}\right)=8.5408$, i.e. $v\left(P_{\mathcal{T}}\right)=v\left(D_{\mathcal{T}}\right)$ (see Fig. 4$)$.

As one can notice in Table 8, the dual algorithm needs roughly half of the time and the number of iterations needed by the primal one to solve the problem. 
Table 8 Performance evaluation for 7 points in $\mathbb{R}^{3}$

\begin{tabular}{|c|c|c|c|c|}
\hline & \multicolumn{2}{|l|}{$\varepsilon_{1}=10^{-4}$} & \multicolumn{2}{|l|}{$\varepsilon_{2}=10^{-8}$} \\
\hline & Primal $(v=10)$ & $\operatorname{Dual}(v=0.055)$ & Primal $(v=10)$ & Dual $(v=0.055)$ \\
\hline CPU time (s) & 0.1871 & 0.0992 & 0.4234 & 0.2042 \\
\hline Number of iterations & 357 & 192 & 955 & 523 \\
\hline
\end{tabular}

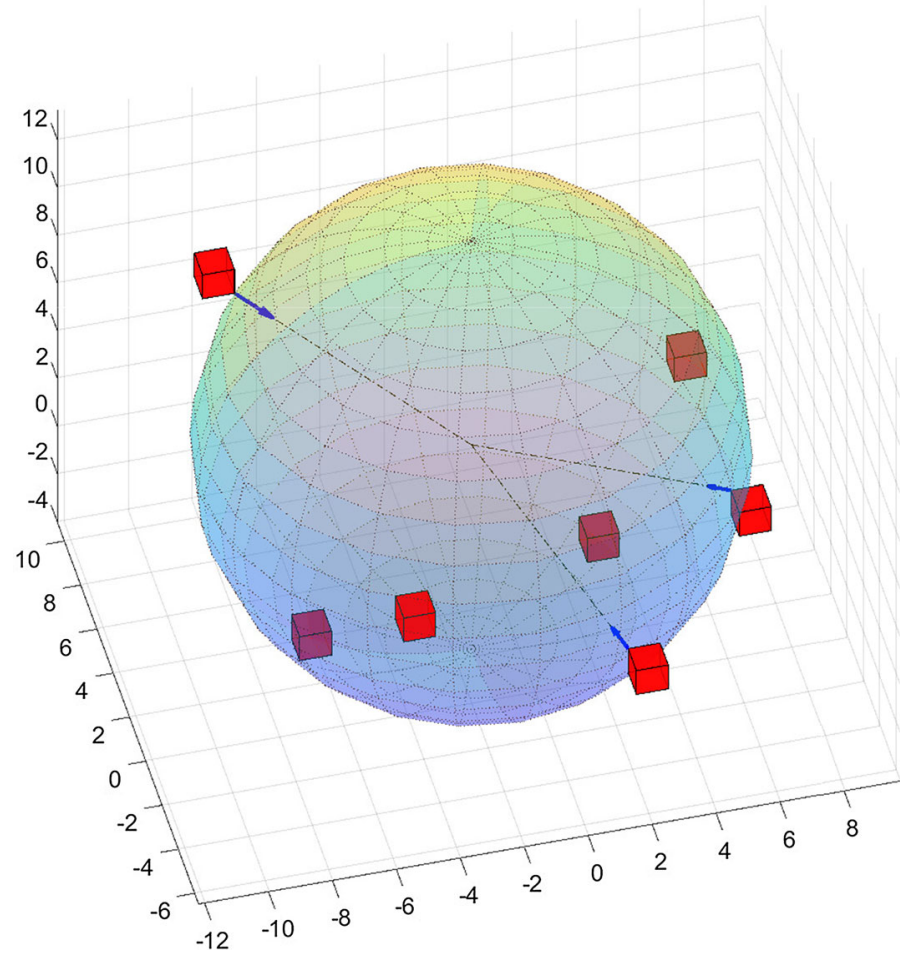

Fig. 4 Visualization of the optimal solutions of the location problem $\left(P_{\mathcal{T}}\right)$ and its dual problem $\left(\widetilde{D}_{\mathcal{T}}\right)$

We close this section by a comparison of the fastest method of the above considered solving strategies, i.e. the dual method, and the one introduced in [1] regarding speed and especially precision in high dimensions.

Example 4.5 We consider the location problem $\left(P_{\mathcal{T}}\right)$ as well as its associated dual one $\left(\widetilde{D}_{\mathcal{T}}\right)$ in the setting where $\Omega_{i}=\left\{x \in \mathbb{R}^{d}:\left\|x-p_{i}\right\|_{\infty} \leq a_{i}\right\}, L_{i}=\left\{0_{\mathbb{R}^{d}}\right\}$ and $\gamma_{C_{i}}=\|\cdot\|$, $i=1, \ldots, n$, and compare the dual method with the numerical algorithm build on the logexponential smoothing technique and Nesterov's accelerated gradient method (log-exp), which was developed in [1] for solving generalized Sylvester problems of the kind of $\left(P_{\mathcal{T}}\right)$.

We implemented this algorithm in MATLAB and used for our numerical tests the same settings as in [1, Remark 5.1] and [1, Example 6.4] (i.e. $\varepsilon=10^{-6}, \widetilde{\varepsilon}=10^{-5}, p_{0}=5$ and $\left.\gamma_{0}=0.5\right)$. We considered four situations where the test results are printed in the Tables 9 , 10,11 and 12. Notice, that in all situations the starting point was the origin and the points $p_{1}, \ldots, p_{n}$ were generated by the command RANDN, while the corresponding radii $a_{1}, \ldots, a_{n}$ 
Table 9 Performance evaluation for 10 points in $\mathbb{R}^{10}$

\begin{tabular}{lll}
\hline & Dual $(v=0.1)$ & $\log -\exp \left(N=35000, v\left(P_{\mathcal{T}}\right)=3.099896\right)$ \\
\hline CPU time (s) & 0.2889 & 55.4856 \\
Number of iterations & 1167 & 32,265 \\
Objective function value & 3.099896 & 3.099896 \\
\hline
\end{tabular}

were given by RAND. As mentioned, we are interested in an analysis regarding the precision of these two methods in such a way that the calculated objective values are exact up to six decimal places, which is especially important in the situation when these calculations are a part of a larger problem where the aim is to reduce rounding errors.

So, to guarantee this desired precision in all four situations we tested the log-exponential smoothing algorithm for various numbers of iterations $N$. For the scenario in Table 9 we received a solution such that the objective value was exact up to six decimal places for $N=35,000$ and saved the calculated solution as the optimal solution $\bar{x}$ to $\left(P_{\mathcal{T}}\right)$, where the corresponding objective value was $v\left(P_{\mathcal{T}}\right)=3.099896$. Then we ran the log-exponential algorithm a second time and noticed the number of iterations, the time needed to generate a solution which is within the maximum bound of $10^{-6}$ from the optimal solution $\bar{x}$ and the associated objective value. Note that, if we reduce the number of iterations in the logexponential smoothing algorithm then the speed to generate a solution such that the objective value is close to the optimal objective value increases, but the accuracy decreases, i.e. then the algorithm fails to calculate a solution such that the objective value is exact up to six decimal places. For the scenarios in the Tables 10,11 and 12 we proceed in the same manner, where the corresponding values for $N, v\left(P_{\mathcal{T}}\right)$ and CPU time are also presented.

For the dual method we set in all situations $N$ to 100,000 and saved the determined solutions for the second run, where then the number of iterations and the CPU time needed to get a solution which is within the maximum bound of $10^{-6}$ were noticed. The corresponding objective values of the dual problem were also recorded.

As you may see in the Tables 9, 10, 11 and 12 the dual method performs again very well regarding speed and also precision in all four situations, which makes it a good candidate not only for problems where precision is of great importance but also for problems in high dimensions, that appear for instance in machine learning. Take also note, that if one has an optimal solution to the dual problem, then the optimal solution to the primal one can be reconstructed by using the formulae given in Remark 3.15.

Remark 4.1 The examples investigated in this section reveal that the origin seems to be a good starting point for running the proposed splitting proximal point method on the dual problem of a given nonlinear minmax location problem. This is actually not very surprising

Table 10 Performance evaluation for 50 points in $\mathbb{R}^{50}$

\begin{tabular}{lll}
\hline & $\operatorname{Dual}(v=0.07)$ & $\log -\exp \left(N=45000, v\left(P_{\mathcal{T}}\right)=6.066194\right)$ \\
\hline CPU time (s) & 7.6268 & 70.3653 \\
Number of iterations & 1956 & 44,173 \\
Objective function value & 6.066194 & 6.066195 \\
\hline
\end{tabular}


Table 11 Performance evaluation for 100 points in $\mathbb{R}^{100}$

\begin{tabular}{lll}
\hline & Dual $(v=0.07)$ & $\log -\exp \left(N=70000, v\left(P_{\mathcal{T}}\right)=9.700309\right)$ \\
\hline CPU time (s) & 104.5634 & 145.2422 \\
Number of iterations & 3003 & 69,163 \\
Objective function value & 9.700309 & 9.700310 \\
\hline
\end{tabular}

Table 12 Performance evaluation for 100 points in $\mathbb{R}^{1000}$

\begin{tabular}{lll}
\hline & Dual $(v=0.01)$ & $\log -\exp \left(N=700000, v\left(P_{\mathcal{T}}\right)=29.528790\right)$ \\
\hline CPU time (s) & 5328.3671 & 7026.1593 \\
Number of iterations & 4017 & 691,412 \\
Objective function value & 29.528790 & 29.528791 \\
\hline
\end{tabular}

when one analyzes the constraints of the dual problems that do not allow (all) the components of the feasible dual solutions to wander far away from the origin.

\section{Conclusions}

We investigate nonlinear minmax location problems (that generalize the classical Sylvester problem) formulated by means of an extended perturbed minimal time function introduced in this paper as well. The motivation to study such problems is not only intrinsic but comes from various areas of research and real life, such as geometry, physics, economics or health management, applications from these fields being mentioned in our paper as possible interpretations of our results. A conjugate duality approach based on rewriting the original problems as multi-composed optimization ones is considered, necessary and sufficient optimality conditions being delivered together with characterizations of the optimal solutions in some particular instances. A parallel splitting proximal point algorithm from [3] is then applied on some concrete location problems and on their duals in MATLAB, delivering optimal solutions to the considered optimization problem faster and with reduced costs than the existing methods in the literature. The tests show that employing the method on the dual is the fastest (and usually also the cheapest) way to solve a given nonlinear minmax location problem. Worth noticing is that this conclusion can be reached regardless of the magnitude of the considered data sets and of the dimension of the involved vectors, suggesting possible employment of the considered method for solving big data problems arising, for instance, in machine learning, by means of support vector techniques. Another idea for future developments of this contribution consists in employing other recent proximal splitting methods for solving nonlinear minmax location problems.

Acknowledgements Open access funding provided by University of Vienna. The authors are grateful to an anonymous reviewer for hints and comments that helped us improve the original version of the paper and for suggesting us a comparison with the algorithm proposed in [1] and to test our method on large data sets with high dimensions.

Open Access This article is distributed under the terms of the Creative Commons Attribution 4.0 International License (http://creativecommons.org/licenses/by/4.0/), which permits unrestricted use, distribution, and repro- 
duction in any medium, provided you give appropriate credit to the original author(s) and the source, provide a link to the Creative Commons license, and indicate if changes were made.

\section{References}

1. An, N.T., Giles, D., Nam, N.M., Rector, R.B.: The log-exponential smoothing technique and Nesterov's accelerated gradient method for generalized Sylvester problems. J. Optim. Theory Appl. 168(2), 559-583 (2016)

2. Bauschke, H.H., Buiy, M.N., Wang, X.: On sums and convex combinations of projectors onto convex sets. arXiv:1802.02287v2 (2018)

3. Bauschke, H.H., Combettes, P.L.: Convex Analysis and Monotone Operator Theory in Hilbert Spaces, CMS Books in Mathematics, 2nd edn. Springer, New York (2017)

4. Berger, A., Grigoriev, A., Panin, A., Winokurow, A.: Location, pricing and the problem of Apollonius. Optim. Lett. 11(8), 1797-1805 (2017)

5. Bounkhel, M.: Subdifferential properties of minimal time functions associated with set-valued mappings with closed convex graphs in Hausdorff topological vector spaces. J. Funct. Spaces Appl. Article ID 707603, 13 pages (2013)

6. Boţ, R.I., Grad, S.-M., Wanka, G.: Duality in Vector Optimization. Springer, Heidelberg (2009)

7. Boţ, R.I., Wanka, G.: The conjugate of the pointwise maximum of two convex functions revisited. J. Glob. Optim. 41(4), 625-632 (2008)

8. Chierchia, G., Pustelnik, N., Pesquet, J.-C., Pesquet-Popescu, B.: Epigraphical projection and proximal tools for solving constrained convex optimization problems. Signal Image Video Process. 9(8), 1737-1749 (2015)

9. Grad, S.-M.: On gauge functions for convex cones with possibly empty interiors. J. Convex Anal. 24(2), 519-524 (2017)

10. Grad, S.-M., Wanka, G., Wilfer, O.: Duality and $\varepsilon$-optimality conditions for multi-composed optimization problems with applications to fractional and entropy optimization. Pure Appl. Funct. Anal. 2(1), 43-63 (2017)

11. Kusraev, A.G., Kutateladze, S.S.: Subdifferentials: Theory and Applications, Mathematics and Its Applications 323. Kluwer, Dordrecht (1995)

12. Meng, L., Peng, L., Wen, C.-F.: Fréchet and proximal subdifferentials of perturbed minimal time functions. J. Nonlinear Convex Anal. 17(9), 1729-1737 (2016)

13. Mordukhovich, B.S., Nam, N.M.: An Easy Path to Convex Analysis and Applications. Morgan and Claypool Publishers, Williston (2014)

14. Mordukhovich, B.S., Nam, N.M.: Limiting subgradients of minimal time functions in Banach spaces. J. Global Optim. 46(4), 615-633 (2010)

15. Mordukhovich, B.S., Nam, N.M.: Subgradients of minimal time functions under minimal requirements. J. Convex Anal. 18(4), 915-947 (2011)

16. Nam, N.M.: Subdifferential formulas for a class of non-convex infimal convolutions. Optimization 64(10), 2213-2222 (2015)

17. Nam, N.M., An, N.T., Salinas, J.: Applications of convex analysis to the smallest intersecting ball problem. J. Convex Anal. 19(2), 497-518 (2012)

18. Nam, N.M., Hoang, N., An, N.T.: Constructions of solutions to generalized Sylvester and FermatTorricelli problems for Euclidean balls. J. Optim. Theory Appl. 160(2), 483-509 (2014)

19. Nam, N.M., Zălinescu, C.: Variational analysis of directional minimal time functions and applications to location problems. Set-Valued Var. Anal. 21(2), 405-430 (2013)

20. Nesterov, Y.: Introductory Lectures on Convex Optimization: A Basic Course, Applied optimization 87. Kluwer, Dordrecht (2004)

21. Nielsen, F., Nock, R.: Approximating smallest enclosing balls with applications to machine learning. Int. J. Comput. Geom. Appl. 19(5), 389-414 (2009)

22. Rockafellar, R.T.: Convex Analysis. Princeton University Press, Princeton (1970)

23. Rockafellar, R.T.: Level sets and continuity of conjugate convex functions. Trans. Am. Math. Soc. 123(1), 46-63 (1966)

24. Wang, J.H., Li, C., Xu, H.K.: Subdifferentials of perturbed distance function in Banach spaces. J. Glob. Optim. 46(4), 489-501 (2010)

25. Wanka, G., Wilfer, O.: A Lagrange duality approach for multi-composed optimization problems. TOP 25(2), 288-313 (2017) 
26. Wanka, G., Wilfer, O.: Duality results for extended multifacility location problems. Optimization 67(7), 1095-1119 (2018)

27. Wanka, G., Wilfer, O.: Duality results for nonlinear single minimax location problems via multi-composed optimization. Math. Methods Oper. Res. 86(2), 401-439 (2017)

28. Wanka, G., Wilfer, O.: Formulae of epigraphical projection for solving minimax location problems, Preprint 2017-5. Faculty of Mathematics, Chemnitz University of Technology (2017)

29. Wilfer, O.: Duality investigations for multi-composed optimization problems with applications in location theory. Ph.D. Thesis, Chemnitz University of Technology (2017)

30. Zălinescu, C.: Convex Analysis in General Vector Spaces. World Scientific, Singapore (2002)

31. Zhang, Y., He, Y., Jiang, Y.: Subdifferentials of a perturbed minimal time function in normed spaces. Optim. Lett. 8(6), 1921-1930 (2014)

Publisher's Note Springer Nature remains neutral with regard to jurisdictional claims in published maps and institutional affiliations. 